\title{
Adaptive Coverage for Future LTE Networks
}

by

\section{Rami Sabouni, MEng., MASc.}

\author{
A dissertation \\ in partial fulfillment of the requirements for the degree of \\ Doctor of Philosophy in Electrical and Computer Engineering
}

Ottawa-Carleton Institute for Electrical and Computer Engineering (OCIECE)

Department of Systems and Computer Engineering

$$
\begin{gathered}
\text { Carleton University } \\
\text { Ottawa, Ontario, Canada }
\end{gathered}
$$

June 2017

Copyright 2017@

Rami Sabouni 


\section{Abstract}

In the past few decades, wireless communications have been growing rapidly and the growth rate is accelerating at a very fast pace. In order to meet this growing demand, researchers are struggling to solve two fundamental problems: (1) providing the spectrum resources needed to support clusters of dense users with very high data rate and (2) supplying the required spectrum at reasonable power requirement level. This thesis first outlines the extent of this capacity-power problem now and in the near future and discusses possible approaches to meet these difficult challenges. We start by introducing a new metric, called Total Energy Per Bit (TEPB), to be used as a measure for greening of the network. Then, a proposed vision of replacing MacroeNBs with a large number of Pico-eNBs to bring the network closer to the users is provided. This naturally leads to casting the problem as an optimization problem with an objective function of minimizing the overall power consumption while supporting user demand with acceptable quality of service. Based on the optimization problem, we propose two algorithms based on the concept of self organizing network (SON) to switch on/off base stations. Simulation results show that both proposed switch on/off algorithms can improve the power requirement of the network while maintaining the required UE throughput by reducing the TEPB significantly. 


\title{
Dedication
}

To:

\author{
$\underline{\text { My dear wife }}$ \\ Mrs. Cassandra Martineau
}

$\underline{\text { My parents }}$

Dr. Abdul-Rahim Sabouni and Mrs. Maissaa Baroudi

$\underline{\text { My daughters }}$

Lara Sabouni and Farah Sabouni 


\section{Acknowledgments}

Many people supported me during the completion of this thesis with criticism, helpful assistance and references. This thesis would have never been possible without them.

I am greatly thankful to my supervisors Prof. Roshdy Hafez and Prof. Marc StHilaire, for their guidance, insights, thoughtful suggestions and continuous support during the course of this research. I am also grateful to my mother Mrs. Maissaa Baroudi and my father Dr. Abdul-Rahim Sabouni, who have provided me with moral and emotional support in my life. Furthermore, I would like to express my gratitude to my other family members and friends who have supported me along the way. In

addition, I would like to express my appreciation to my colleagues, secretaries and personnel in the Department of System and Computer Engineering and Carleton University without whom this work would not have been possible.

Also, I would like to thank my father in-law and mother in-law for their caring, and inspiration. Finally, I would like to express my deepest thanks to my wife Mrs. Cassandra Martineau for her love, patience and persistent support. 


\section{Table of Contents}

Abstract

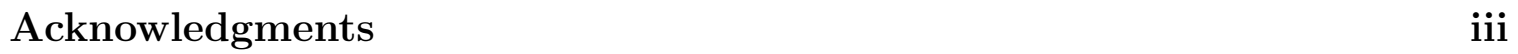

Table of Contents iv

List of Tables viii

List of Figures $\quad$ ix

List of Acronyms xii

List of Symbols $\quad$ xvii

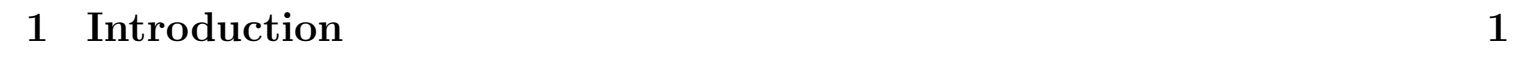

1.1 Problem Statement . . . . . . . . . . . . . . . . . . . . . . . 1

1.2 Research Contributions . . . . . . . . . . . . . . . . . . . . . . 6

1.3 Publications . . . . . . . . . . . . . . . . . . . . . . . . . . . 8

1.4 Thesis Organization . . . . . . . . . . . . . . . . . . . . 8

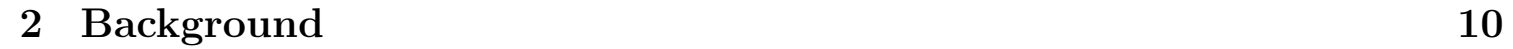

2.1 Introduction to LTE $\ldots \ldots \ldots \ldots$. . . . . . . . . . . . . . 10

2.2 LTE Network Architecture . . . . . . . . . . . . . . . . . . . . . . . . 13

$2.2 .1 \quad$ Evolved Node B (eNB) . . . . . . . . . . . . . . 13 
$2.2 .2 \quad$ Mobility Management Entity (MME) _ . . . . . . . . . 15

$2.2 .3 \quad$ Serving Gateway $(\mathrm{S}-\mathrm{GW}) \quad \ldots \ldots \ldots \ldots \ldots$

2.2 .4 Packet Data Network Gateway (P-GW) . . . . . . . 16

$2.2 .5 \quad$ Element Management System/Network Management System $(\mathrm{EMS} / \mathrm{NMS})$

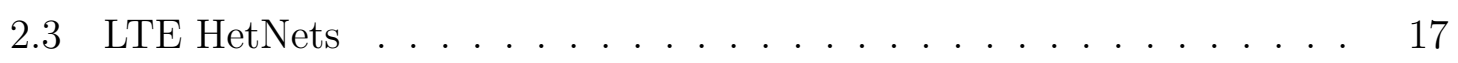

$2.4 \quad$ LTE Power Consumption . . . . . . . . . . . . . . . . . . 18

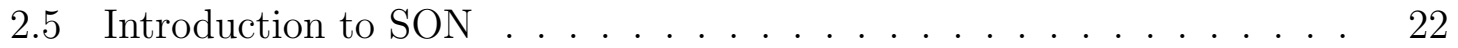

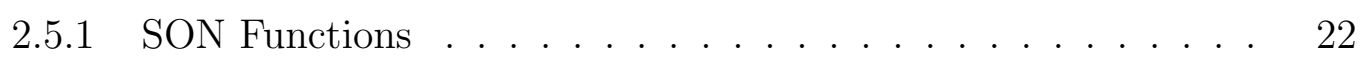

2.5 .2 SON Architecture . . . . . . . . . . . . . . 24

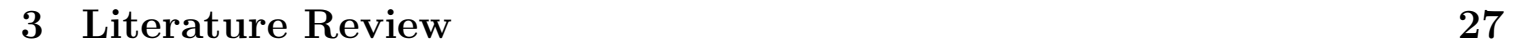

3.1 Introduction . . . . . . . . . . . . . . . . . . . . . . . 27

3.2 Energy Saving Approach for LTE networks . . . . . . . . . . . . 28

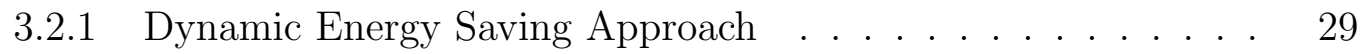

$3.2 .2 \quad$ Static Energy Saving Approaches _ . . . . . . . . . . . . 32



3.3 Heterogeneous Network Deployment. . . . . . . . . . . . . . . . 36

$3.3 .1 \quad$ Macro-Pico-eNB Deployment $\ldots \ldots \ldots \ldots$. . . . . . . 37

$3.3 .2 \quad$ Macro-Micro-eNB Deployment. . . . . . . . . . . . . . . . 40

$3.3 .3 \quad$ Macro-Femto-eNB Deployment . . . . . . . . . . . . . . 41

3.4 Summary $\ldots \ldots \ldots \ldots \ldots \ldots$

\begin{tabular}{|lll|}
4 & Proposed Vision and Algorithms & 48 \\
\hline
\end{tabular}

4.1 Overview of Proposed Vision . . . . . . . . . . . . . . . . . . . 48

$4.1 .1 \quad$ Proposed Metric $\ldots \ldots \ldots \ldots$

4.1 .2 Implementation of the Proposed Vision . . . . . . . . . . 57

$4.1 .3 \quad$ Backhaul and Handover Challenges . . . . . . . . . . . 57 
4.2 Optimization Problem Formulation $\ldots \ldots \ldots \ldots$

4.3 Design Approach $\ldots \ldots \ldots$. . . . . . . . . . . . . . . . . . . . . . . 61

4.3 .1 LTE Propagation Path-Loss Models . . . . . . . . . . . . . . 62

4.3 .2 SINR Calculation . . . . . . . . . . . . . . . . . . . . 64

$4.3 .3 \quad$ CQI Calculation $\ldots \ldots \ldots \ldots \ldots \ldots$

4.3 .4 Network Model . . . . . . . . . . . . . . . . . . . . . 66

4.3 .5 Complexity of the network model . . . . . . . . . . . 69

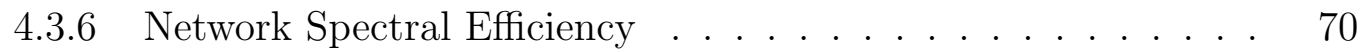

$4.3 .7 \quad$ LTE Frame Structure . . . . . . . . . . . . . . . . . 70

4.4 Proposed Switch On/Off Algorithms … . . . . . . . . . 71

4.4 .1 The Centralized Switch On/Off Algorithm . . . . . . . . . 72

4.4 .2 The Enhanced Centralized Switch On/Off Algorithm . . . . 78

$4.5 \quad$ UE Distribution and Mobility Model . . . . . . . . . . . . . . . 83

4.5 .1 Gaussian Distribution . . . . . . . . . . . . . . 83

4.5 .2 Uniform Distribution . . . . . . . . . . . . . . . . . . . 84

$4.5 .3 \quad$ Manhattan Mobility Model . . . . . . . . . . . . . . 84

\begin{tabular}{|lll}
\hline 5 & Simulation Results and Analysis & 87
\end{tabular}

$5.1 \quad$ System-Level Simulator . . . . . . . . . . . . . . . . . . . . . . 87

5.1 .1 CSA Simulation . . . . . . . . . . . . . . . . . . . . . . 89

$5.1 .2 \quad$ ECSA Simulation . . . . . . . . . . . . . . . . . . . . 89

5.2 CSA Simulation Results and Analysis . . . . . . . . . . . . . 90

$5.2 .1 \quad$ Simulation Setup . . . . . . . . . . . . . . . . 90 90

5.2 .2 Evaluation of the Range Effect on the CSA . . . . . . . . 91

5.2 .3 Evaluation of the Effect of UE Number on the CSA . . . . . 98

$5.3 \quad$ ECSA Simulation Results and Analysis . . . . . . . . . . . . . . 101

5.3 .1 Simulation Setup . . . . . . . . . . . . . . . . . . 101 
5.3 .2 Sample Simulation Run . . . . . . . . . . . . . . . . . 102

5.3 .3 Evaluation of the Effect of UE Number on the ECSA . . . . 105

5.3 .4 CSA vs. ECSA $\ldots \ldots \ldots \ldots \ldots \ldots$

$5.3 .5 \quad$ ECSA vs. Two Algorithms from Literature. . . . . . . . . . 109

6 Conclusion and Future Work 114

6.1 Conclusion . . . . . . . . . . . . . . . . . . . . . . . . . 114

6.1 .1 The Road to 5G Networks . . . . . . . . . . . . . . . . . . 116

6.2 Future Work . . . . . . . . . . . . . . . . . . . . . . 118

6.2 .1 Test the ECSA in a Real Network Setup . . . . . . . . . . . 119

6.2 .2 Implement a Distributed Switch On/Off Algorithm . . . . . . 119

6.2.3 Include Backhauling in the Switch On/Off Algorithm . . . . . 120

$6.2 .4 \quad$ Adding New Pico-eNB When Needed . . . . . . . . . . . . . 121

\begin{tabular}{|ll}
\hline A SINR Distribution Calculation Proof & 123
\end{tabular}

\begin{tabular}{ll}
\hline References & 127
\end{tabular} 


\section{List of Tables}

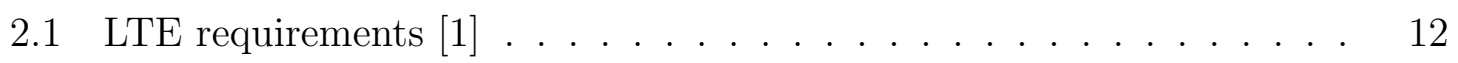

2.2 Power consumption of different parts of Macro-eNB [2] $\ldots \ldots \ldots .20$

2.3 Power consumption of different types of eNBs $[2] \ldots \ldots \ldots \ldots$

$3.1 \quad$ Summary of related work for HoNets $\ldots \ldots \ldots \ldots \ldots$

3.2 Summary of related work for HetNets . . . . . . . . . . . . 47

$4.1 \quad$ Lookup table for mapping SINR estimate to CQI index $|3|] \ldots \ldots$

4.2 Number of resource blocks for different bandwidths [3] $\ldots \ldots \ldots . .71$

4.3 Processing time of the CSA for 500 - 1500 UE in the network. . . . . 80

$5.1 \quad$ Simulation parameters to evaluate the CSA $\ldots \ldots \ldots \ldots . \ldots 92$

5.2 Power consumption for all three scenarios. . . . . . . . . . . 98

$5.3 \quad$ Simulation parameters to evaluate the ECSA $\ldots \ldots \ldots \ldots$

5.4 Simulation parameters to compare ECSA with greedy-drop and greedyadd algorithms . . . . . . . . . . . . . . . . . . . . . 112 


\section{List of Figures}

1.1 Network operation a) without; b) with SON functions. . . . . . . . . 3

1.2 Effects of traffic types on wireless coverage . . . . . . . . . . . . 5

2.1 E-UTRAN network architecture . . . . . . . . . . . . . . . . 11

2.2 LTE architecture . . . . . . . . . . . . . . . . . . . . . . . . . . 14

2.3 Heterogeneous networks consist of Macro, Micro, Pico, and Femto eNBs . . . . . . . . . . . . . . . . . . . 18

2.4 Simplified block diagram of an eNB. . . . . . . . . . . . . . 20

2.5 Pico-eNB power consumption breakdown for different components 4 4] 21

2.6 Centralized SON architecture . . . . . . . . . . . . . . . . . . . . . . 24

2.7 Distributed SON architecture . . . . . . . . . . . . . . . . 26

4.1 Proposed vision: LTE network with only Pico-eNBs that can be switched on/off . . . . . . . . . . . . . . . . . . . . . 51

4.2 Network power consumption vs network throughput for 500 UE . . . $\quad 55$

4.3 Effect of cell splitting on TEPB . . . . . . . . . . . . . . . . 55

4.4 Network setup with 1 and 64 eNBs, also showing the range of each eNB 56

4.5 LTE system model with various possible types of Pico-eNB backhauling 58

4.6 LTE frame structure . . . . . . . . . . . . . . . . . . . . . . . . . . . 71

4.7 The centralized switch on/off algorithm . . . . . . . . . . . . . 73

4.8 Initial network . . . . . . . . . . . . . . . . . . . . . . . . . . . . . 76 
4.9 Network after centralized switch on/off algorithm is applied and Pico-

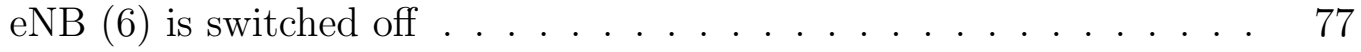

4.10 CSA Pico-eNB grouping example (groups 1 to 3 ) . . . . . . . . . . . 79

4.11 CSA Pico-eNB grouping example (groups 4 to 7) . . . . . . . . . . . 79

4.12 The enhanced centralized switch on/off algorithm . . . . . . . . . . . 81

4.13 Network setup and UE movement . . . . . . . . . . . . . . . . . . . 85

$5.1 \quad$ Simulation setup to calculate the effect of distance between the centre of the network and UE's grouping . . . . . . . . . . . . . . . . . . . 91

5.2 Sample simulation to simulate 500 UE moving from centre to the upper edge of the network . . . . . . . . . . . . . . . . . . . . . . . . . 94

$5.3 \quad$ TEPB of Macro-eNB vs all Pico-eNBs on for UE moving from centre to the upper edge of the network f . . . . . . . . . . . . . . . . . . 96

5.4 TEPB of Macro-eNB vs all Pico-eNBs on for UE moving from centre to the right edge of the network . . . . . . . . . . . . . . . . . 96

5.5 Simulation results for UE's centre of distribution moving from centre

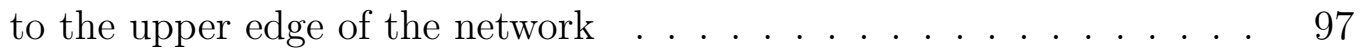

5.6 Simulation results for UE's centre of distribution moving from centre to the right edge of the network . . . . . . . . . . . . . . . . . . . . . 97

5.7 Simulation to simulate the effect of the number of UE on the CSA . . 99

5.8 Simulation results for 500, 1000 and 1500 UE at the centre of the network 100

$5.9 \quad$ E-UTRAN network architecture with only Pico-eNBs . . . . . . . . . 102

5.10 Simulation setup for evaluating the effect of the number of UE on the ECSA . . . . . . . . . . . . . . . . . . 103

5.11 Simulation sample for the ECSA for $500 \mathrm{UE}$. . . . . . . . . . . . 104

5.12 Simulation results for $500,1000,1500,2000$ UE at the centre of the network . . . . . . . . . . . . . . . . 106 
5.13 Simulation setup for evaluating the performance of CSA and ECSA for $500 \mathrm{UE} \ldots \ldots \ldots \ldots \ldots$

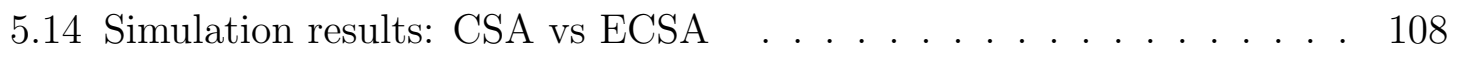

5.15 Comparison of the \% of Pico-eNBs switched off in the network between the ECSA, the greedy-drop algorithm and the greedy-add algorithm . 111

5.16 Comparison of TEPB of the network between the ECSA, the greedydrop algorithm and the greedy-add algorithm . . . . . . . . . . . . . 112 


\section{List of Acronyms}

\begin{tabular}{ll}
\hline Acronyms & Definition \\
\hline \hline 16-QAM & 16-Quadrature Amplitude Modulation \\
2G/3G & Second/Third-Generation \\
3GPP & 3rd Generation Partnership Project \\
$4 \mathrm{G}$ & Fourth-Generation \\
$5 \mathrm{G}$ & Fifth-Generation \\
AAA & Authentication, Authorization and Accounting \\
BDMA & Beam Division Multiple Access \\
BILP & Binary Integer Linear Programming \\
BLR & Block Error Rate \\
& Codase Station \\
&
\end{tabular}




\begin{tabular}{|c|c|}
\hline $\mathrm{COC}$ & Cell Outage Compensation \\
\hline CQI & Channel Quality Indicator \\
\hline CSA & Centralized Switch on/off Algorithm \\
\hline $\mathrm{D} 2 \mathrm{D}$ & Device-to-Device \\
\hline DHCP & Dynamic Host Configuration Protocol \\
\hline E-UTRAN & Evolved Universal Terrestrial Radio Access Network \\
\hline $\mathrm{EB}$ & Exabytes \\
\hline ECSA & Enhanced Centralized Switch on/off Algorithm \\
\hline EMS/NMS & Element Management System/Network Management System \\
\hline eNB & Evolved NodeB \\
\hline $\mathrm{EPC}$ & Evolved Packet Core \\
\hline FCAPS & Fault, Configuration, Accounting, Performance, Security \\
\hline FDD & Frequency Division Duplexing \\
\hline FDMA & Frequency-Division Multiple Access \\
\hline GSM & Global System for Mobile \\
\hline HARQ & Hybrid Automatic Repeat Request \\
\hline $\mathrm{HD}$ & High Definition \\
\hline HetNet & Heterogeneous Network \\
\hline HoNet & Homogeneous Network \\
\hline
\end{tabular}




\begin{tabular}{|c|c|}
\hline ICI & Inter Cell Interference \\
\hline ICIC & Inter-Cell Interference Coordination \\
\hline ICT & Information and Communications Technology \\
\hline IID & Independent and Identically Distributed \\
\hline IP & Internet Protocol \\
\hline ICD & Inter-Site Distance \\
\hline LoS & Line-of-Sight \\
\hline LTE & Long Term Evolution \\
\hline MAC & Medium Access Control \\
\hline MCS & Modulation and Coding Scheme \\
\hline MIMO & Multiple-Input Multiple-Output \\
\hline MME & Mobility Management Entity \\
\hline NAS & Non-Access Stratum \\
\hline NLoS & Non Line of Sight \\
\hline NP-Hard & N-deterministic Polynomial-time Hard \\
\hline O\&M & Operation and Maintenance \\
\hline OFDM & Orthogonal Frequency Division Multiplexing \\
\hline OFDMA & Orthogonal FDMA \\
\hline $\mathrm{PDCCH}$ & Physical Downlink Control Channel \\
\hline
\end{tabular}




\begin{tabular}{|c|c|}
\hline PPP & Poisson Point Process \\
\hline QoS & Quality of Service \\
\hline QPSK & Quadrature Phase Shift Keying \\
\hline $\mathrm{RB}$ & Resource Block \\
\hline ROI & Region Of Interest \\
\hline RRM & Radio Resource Management \\
\hline S-GW & Serving Gateway \\
\hline SC-FDMA & Single-carrier FDMA \\
\hline SINR & Signal-to-Interference plus Noise Ratio \\
\hline SIR & Signal-to-Interference Ratio \\
\hline SNR & Signal-to-Noise Ratio \\
\hline $\mathrm{SON}$ & Self-Organizing Network \\
\hline TA & Tracking Area \\
\hline TB & Transport Block \\
\hline TBS & Transport Block Size \\
\hline TDD & Time Division Duplexing \\
\hline TEPB & Total Energy Per Bit \\
\hline TTI & Transmission Time Interval \\
\hline UAV & Unmanned Aerial Vehicle \\
\hline
\end{tabular}




$\begin{array}{ll}\text { UE } & \text { User Equipment } \\ \text { UMTS } & \text { Universal Mobile Telecommunications System } \\ \text { VoIP } & \text { Voice over IP } \\ \text { WiMAX } & \text { Worldwide Interoperability for Microwave Access }\end{array}$




\section{List of Symbols}

\begin{tabular}{|c|c|}
\hline Symbols & Definition \\
\hline$\eta$ & Network average spectral efficiency in $\left(\right.$ bit $\left./ \mathrm{s} / \mathrm{Hz} / \mathrm{m}^{2}\right)$ \\
\hline$\gamma$ & SINR threshold \\
\hline$\lambda_{b} P_{a}$ & Density of active eNBs \\
\hline$\lambda_{b}$ & Pico-eNB density \\
\hline$\lambda_{u}$ & User equipment density \\
\hline$\mu_{i}$ & Mean of each random variable \\
\hline$\mu$ & Constant related to the cell size distribution \\
\hline$\rho$ & ENB-UE density ratio \\
\hline$\sigma^{2}$ & Variance of the distribution \\
\hline$\sigma_{i}$ & Standard deviation of each random variable \\
\hline$\theta$ & UE movement direction \\
\hline$a(t)$ & Current acceleration in $\mathrm{m} / \mathrm{s}^{2}$ \\
\hline$a$ & Constant equal to $\frac{\ln 10}{10}$ \\
\hline
\end{tabular}




\begin{tabular}{|c|c|}
\hline$A$ & Network area in $m^{2}$ \\
\hline$B W$ & Network bandwidth in $H z$ \\
\hline$c$ & Constant equal to $10^{(140.7 / 10)}$ \\
\hline$d$ & Transmitter-receiver separation in $\mathrm{km}$ \\
\hline$E_{b}$ & Energy per bit \\
\hline$e N B s_{o n}$ & Set of all currently active eNBs \\
\hline$F_{s}$ & Log normal shadow fading with standard deviation $10 \mathrm{~dB}$ \\
\hline$F$ & $\begin{array}{l}\text { Sum of log-normally independent and identically dis- } \\
\text { tributed (iid) random variables }\end{array}$ \\
\hline$G_{\text {antenna }}$ & Antenna gain in $\mathrm{dBi}$ \\
\hline$G_{e N B}$ & ENB antenna gain in $\mathrm{dBi}$ \\
\hline$G_{U E}$ & $\mathrm{UE}$ antenna gain in $\mathrm{dBi}$ \\
\hline$I$ & Set of eNB that are installed in the network \\
\hline$j$ & Index of the serving eNB \\
\hline$J$ & Set of UE in the network \\
\hline$k$ & Number of surrounding eNBs \\
\hline$m_{f}$ & Mean of $F$ \\
\hline$a x$ & Distribution maximum values \\
\hline
\end{tabular}




\begin{tabular}{|c|c|}
\hline$N_{c o m b}$ & Number of possible eNB combinations \\
\hline$N_{e N B s}$ & Number of eNBs in the network \\
\hline$N_{T B}$ & Number of Transport Blocks / Subframe \\
\hline$N_{0}$ & Noise density \\
\hline$N_{i}$ & $\begin{array}{l}\text { Maximum number of UE that can be served by Pico-eNB } \\
i \in I\end{array}$ \\
\hline$N_{r}$ & Number of rings in the network \\
\hline$N$ & Number of eNB groups \\
\hline$N e t_{t h r}$ & Network throughput \\
\hline$P_{0}$ & $\begin{array}{l}\text { Probability that a randomly chosen eNB does not have any } \\
\text { UE in its coverage }\end{array}$ \\
\hline$P_{\text {consumed }}$ & Total power consumed by the network in Watts \\
\hline$P_{\text {out }}$ & Network outage probability \\
\hline$P_{r x}$ & Received power from the serving eNB \\
\hline$P_{t x}$ & Transmitted power from the serving eNB \\
\hline$P_{a}$ & Probability that an eNB is active \\
\hline$P_{i}$ & Power consumed per eNB in Watts for eNB $i \in I$ \\
\hline$P_{s}$ & $\begin{array}{l}\text { Probability that a randomly chosen UE is successfully as- } \\
\text { signed a RB }\end{array}$ \\
\hline$P L_{\text {Macro }}$ & Macro-eNB Path-Loss \\
\hline
\end{tabular}




\begin{tabular}{|c|c|}
\hline$P L_{\text {Pico }}$ & Pico-eNB Path-Loss \\
\hline$P L$ & Path-Loss from the desired eNB \\
\hline$R_{0}$ & Shannons capacity limit \\
\hline$R_{a}$ & Network area spectral efficiency in bits/sec/Hz/unit-area \\
\hline$R_{j}$ & $\begin{array}{l}\text { Number of successfully transmitted bits per UE for UE } \\
j \in J\end{array}$ \\
\hline$R_{u}$ & $\begin{array}{l}\text { Average spectral efficiency per UE in bits/sec/Hz/unit- } \\
\text { area }\end{array}$ \\
\hline$s_{f}$ & Standard deviation of $F$ \\
\hline Sfps & Subframes / Sec \\
\hline$t^{*}$ & Time in sec \\
\hline$T_{0}$ & Thermal noise \\
\hline$T$ & Time interval in $s e c$ \\
\hline$T B S$ & Transport Block Size (bits / Subframe) \\
\hline$T E P B$ & Total energy per bit \\
\hline Throughput & Average network throughput in Mbps \\
\hline$v_{\max }$ & Maximum speed \\
\hline$v_{n e w}$ & Target speed in $\mathrm{m} / \mathrm{s}$ \\
\hline$v(t)$ & Current speed in $\mathrm{m} / \mathrm{s}$ \\
\hline
\end{tabular}


Pico-eNB is off, otherwise 1

Z Percentage of network outage 


\section{Chapter 1}

\section{Introduction}

In this chapter, we present the problem statement that motivated the development of this research. Then, we list the research contributions and the significance of each one. Also, we provide list of publications produced from this thesis. Finally, we explain the thesis organization.

\subsection{Problem Statement}

In the last few years, Long Term Evolution (LTE) networks have been growing rapidly. The number of users and data traffic in LTE networks have increased dramatically. Network operators are constantly trying to satisfy user requirements while reducing capital expenditures/operational expenditures (CAPEX/OPEX). Therefore, the trend is moving towards green networks, networks that are environmentally sustainable and use energy efficiently. The main source of energy consumption in mobile networks is from the base stations. Therefore, to achieve green cellular networks it is crucial to improve the energy efficiency of the evolved NodeBs (eNBs).

In current LTE networks, each eNB transmits data to user equipment (UE) connected to it. The cell size is defined by the area in which the UE can receive data from the eNB connected to it. At the network planning stage, network operators usually 
fix cell sizes and capacities based on estimated peak traffic load, although traffic load in LTE networks can have spatial and temporal fluctuations during the day due to UE movement and different mobile applications. For example, the traffic load during daytime in a downtown area is relatively heavy compared to a residential area, while the opposite maybe true in the evening. If LTE network capacity is planned based on peak traffic load, there are always light and heavy traffic areas in the network. Because of that, any static network planning will not be optimal as traffic fluctuates.

Traffic fluctuation in LTE networks can have a more severe impact with the increasing number of eNBs, since there is a great amount of underutilization in dense eNB networks during off-peak hours. Therefore, that makes network planning and eNB deployment even harder. When the traffic increases higher than the planned capacity in some parts of the network, some UE will experience a decrease in quality of service (QoS). Meanwhile, other parts of the network might have a lighter traffic, which opens the door for load balancing techniques [5].

Operational efficiency being crucial for mobile networks, operators are in need of advanced technologies to reduce the OPEX of LTE networks. The self-organizing network (SON) concept was introduced by the 3rd Generation Partnership Project (3GPP). Figure 1.1 shows a comparison between network operation with the conventional mechanism (which relies on human intervention or service tools) and SON functions. The use of SON enables easier deployment of new eNBs and reduces human intervention. Network optimization is one of the key features of SON. In second and third generations $(2 \mathrm{G} / 3 \mathrm{G})$ mobile networks, a collection of standardized procedures has been defined for wireless network planning and optimization. For mobile networks, network optimization involves, among other things: base station maintenance, signaling, adjustment, data collection, and functions to improve coverage and reduce interference. 




Figure 1.1: Network operation a) without; b) with SON functions.

Future mobile communication systems will be composed of a flexible service environment comprised of network operators, service providers, software developers, customers, etc. Business models in LTE networks will be cross-linked abundantly and provide a wider range of market. The transmission bandwidth and QoS of mobile networks have been greatly improved with the introduction of LTE networks. New wireless applications and services have been developed as a result, causing an increase in data rate requirements. Also, future mobile networks are required to be cost effective and easy to deploy. The requirements of future LTE networks will be higher than the early stage of their deployment because of the continuous increase in customers' expectations regarding coverage and QoS. To reduce CAPEX/OPEX, more efficient algorithms have to be implemented in future LTE networks [6].

The current LTE network infrastructure utilizes large cells (Macro-eNBs). At 2.6 GHz carrier frequency, the range of each cell can be large (i.e. a few kilometers). Unfortunately, in order for LTE networks to provide the promised high data rate (1 Gbps), the coverage area must be smaller.

To understand this, suppose we transmit audio and high definition (HD) video 
signals with the same transmitter. If we assume that audio was sent at a rate of 10 kbps while the video was sent at rate of $400 \mathrm{kbps}$, and furthermore, assume that both signals encounter the same bit error and are received with acceptable quality. Which means that both signals reach the receiver with the same signal-to-noise ratio $E_{b} / N_{0}$ (energy per bit divided by the noise density). Considering the noise density $\left(N_{0}\right)$ is the same, the energy per bit $\left(E_{b}\right)$ for both signals should be the same. For a time period of $T$ seconds, the received audio energy is $E_{a}=1000 \times E_{b} \times T$, while the received video energy is $E_{v}=400,000 \times E_{b} \times T$. The energy can be converted into power by dividing by $T$. Therefore, the required received power ratio of the two signals is $P_{v} / P_{a}=400$. As such, we must either transmit the video signal at higher power or reduce the cell range for the video compared with the audio signal. In the previous example, the path-loss for the video signal should be about $26.02 d B$ (10 $\left.\log _{10} 400\right)$ less than the path-loss of the audio signal.

That path-loss difference can be converted into a possible change in the transmission distance for the same transmitted power, although increasing the transmit power is not always possible or practical. Figure 1.2 shows the effect of different traffic types on wireless coverage. The higher the bit-rate, the smaller the cell range, which means, the cell tower placements that were used for audio signal coverage are no longer suitable for video signals or data intensive applications. This is an approximation of the complex process of dimensioning cellular networks and scheduling traffic types.

In order for LTE networks to meet the growing demand, two fundamental problems need to be solved:

\section{Providing the spectrum resources needed to support clusters of dense users with very high data rate:}

As explained in the previous example, in the near future, all network operators will have to reduce cell ranges to achieve the required high data rate. This 


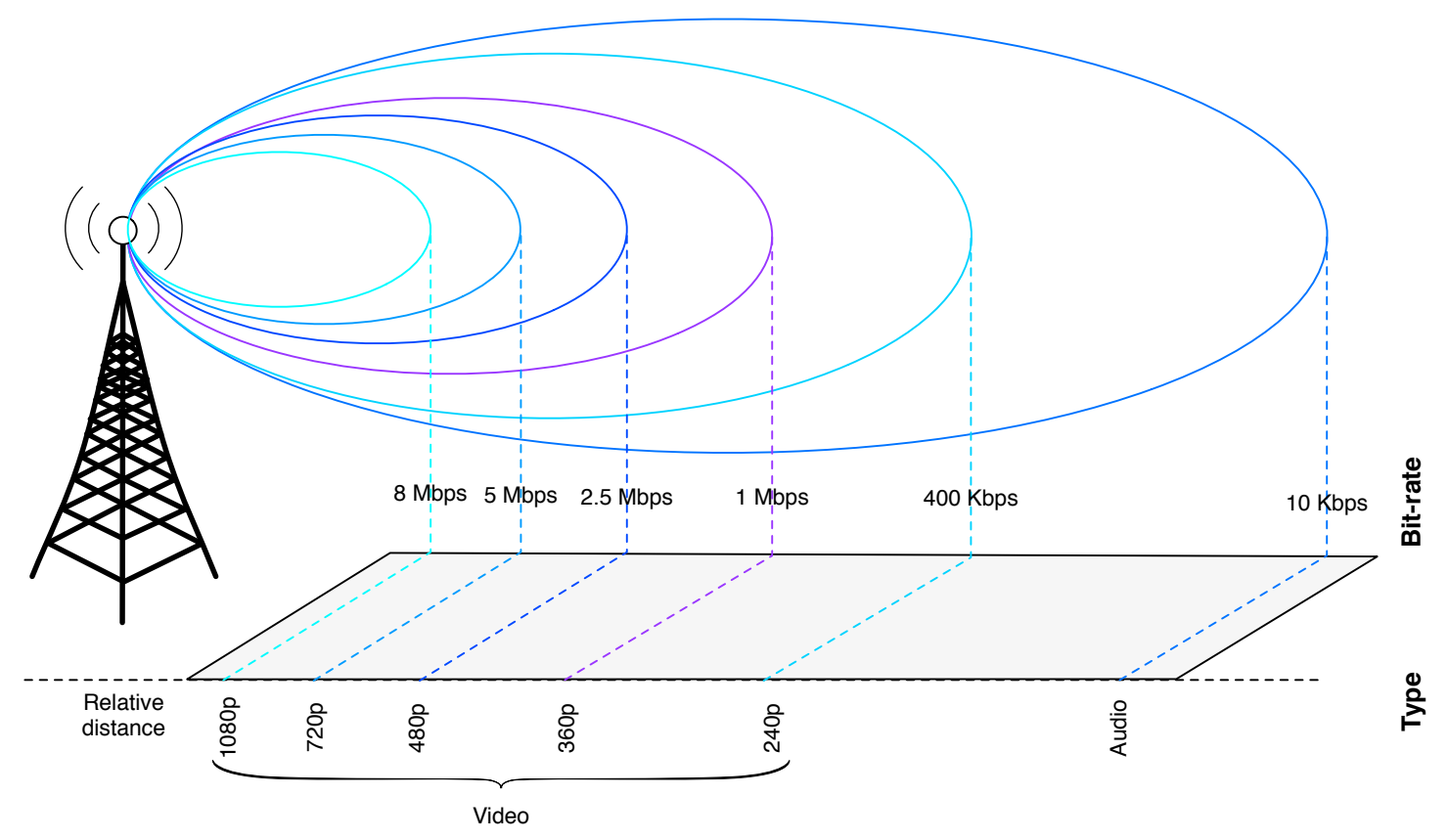

Figure 1.2: Effects of traffic types on wireless coverage

means that more eNBs will have to be deployed and that sophisticated tools will have to be developed and used to deal with the management of all these new network elements.

\section{Supplying the required spectrum at reasonable power consumption} level:

The increase in number of eNBs contributes to a major portion of power consumption of LTE networks, which also results in an increase in the impact of carbon emissions on the environment. Network operators are always searching for ways to reduce power consumption of wireless access networks to provide a greener network. When an eNB is running, the power consumption of signal processors, amplifiers and air conditioner takes up to $60 \%$ of the total power consumption [2]. Therefore, changing transmit power or switching off part of the eNB may have a marginal effect on power consumption. To save energy, 
eNB switching on/off algorithms need to be implemented to provide UE with the desired QoS while reducing the power consumption of the network. Therefore, strategies where eNBs can be autonomously switched on/off should also be part of the future vision.

\section{$1.2 \quad$ Research Contributions}

The main contribution of the current research is to develop a novel approach that will enable LTE networks to provide users with very high data rates where it is needed with minimum power consumption. More specifically, the contributions of this thesis are summarized as follows:

Contribution 1: We introduce a new metric, called total energy per bit (TEPB), to measure the greening of the network.

Significance: With network operators' effort to provide a higher data rate to UE, the number of eNBs in the network is increasing. Network operators can observe a network area with a large number of cells that share the same bandwidth over a long period of time and measure how much power is used in order to deliver a certain number of bits to the users. Therefore, just looking at the data rate or power consumption alone does not provide a clear picture of the performance of the network. The proposed metric (TEPB) can be used to evaluate/compare different algorithms and measure the greening of any network.

Contribution 2: We propose a new vision where all Macro-eNBs are replaced with a large number of Pico-eNBs that cover the same area as the Macro-eNBs.

Significance: In order for Macro-eNBs to deliver high data rates to every spot that it covers, higher transmission power is needed, which is not practical. Another way to accomplish the same is to reduce the distance between the UE 
and the eNB. This can be achieved by cell splitting large cells, which can be done by replacing current Macro-eNBs with a large number of Pico-eNBs.

Contribution 3: We design two centralized switch on/off algorithms that determine which Pico-eNBs should be switched on/off in order to minimize the TEPB while guaranteeing quality of service.

Significance: An LTE network can be controlled with a switch on/off algorithm to decide which Pico-eNB to switch on/off by considering some QoS key factors for Pico-eNBs in LTE networks. These factors, within a certain PicoeNB, include the total data rate delivered to UE, the number of dropped UE, the power consumption and the received interference signal from nearby PicoeNBs. It is known that switching eNBs to find the optimal network setup is a difficult combinatorial problem. Since this problem is NP-hard [7] [8], this thesis proposes two implementable heuristic switch on/off algorithms that consider the main characteristics of LTE networks.

The proposed switch on/off algorithms can help network operators change network configuration by allocating power and coverage to match UE traffic demand at a given time during the day and from day to day. Adding a large number of Pico-eNBs increases the power consumption and the management complexity of the network. To that end, the proposed switch on/off algorithms helps by minimizing the number of Pico-eNBs on while maintaining the required QoS. It turns out that once the algorithms are executed, the number of switched on Pico-eNBs can track the network traffic very well. Multiple simulations were constructed to test the performance of the proposed algorithms. A test was also performed to determine to what extent the proposed vision can be applied. 


\subsection{Publications}

In this section, we list all the publications that were derived from the research work that was carried on as part of this thesis.

- Rami Sabouni, Roshdy M. Hafez, and Marc St-Hilaire, "Adaptive Coverage for Future LTE Networks", under 2nd round of review in IEEE Transaction on Wireless Communications, (submission: 16 September 2016, 1st review submission: 30 January 2017).

- Rami Sabouni, Roshdy M. Hafez, and Marc St-Hilaire, "A centralized algorithm for adaptive coverage in next generation cellular networks", 6th International Conference on the Network of the Future (NOF 2015), Montreal, QC, Canada, pages 1-6, September 2015.

- Rami Sabouni, Roshdy M. Hafez, and Marc St-Hilaire, "Adaptive coverage for high data rate LTE networks", International Conference on Computing, Networking and Communications (ICNC 2014), Honolulu, Hawaii, USA, pages 303-307, February 2014.

\subsection{Thesis Organization}

The rest of this thesis is organized as follows.

Chapter 2 provides a background on related topics including LTE network architecture, heterogeneous networks (HetNets), LTE power consumption and LTE SON, to make it is easier for the reader to understand the content of this thesis.

Chapter 3 gives an overview of the related work in the literature, which includes different types of energy saving approaches for LTE networks and heterogeneous networks in the literature. 
Chapter 4 presents the design approach and path-loss models that are used in this thesis. Also, two switch on/off algorithms were also discussed in this chapter. These two switch on/off algorithms were formulated as an optimization problem.

Chapter 5 presents the performance evaluation of the two switch on/off algorithms proposed in Chapter 4. The algorithms are evaluated by changing different parameters such as UE distribution, number of UE, and number of eNB in the network.

Chapter 6 is dedicated to the research's main conclusions and identifying the possible future work for this thesis. 


\section{Chapter 2}

\section{Background}

In this chapter, we provide background information on related topics that are discussed in this thesis. First, we present an introduction to LTE networks including the main components of its architecture and HetNets. Then, the power consumption of different types of eNBs is reviewed followed by an introduction to the concept of self organizing networks in LTE.

\subsection{Introduction to LTE}

The recent increase in demand for mobile data and development of new mobile applications, such as online gaming and mobile TV, have motivated the $3 \mathrm{GPP}$ to work on LTE, which was introduced in 3GPP Release-8. With the emergence of internet protocol (IP) data traffic, LTE is scheduled to provide support for IP-based traffic with end-to-end QoS. Voice traffic will be supported as voice over IP (VoIP), enabling better integration with other multimedia services.

3GPP specified that LTE consists of two core elements of the evolved universal mobile telecommunications system (UMTS) system architecture: evolved universal

terrestrial radio access network (E-UTRAN) and evolved packet core (EPC) [9] [10]. LTE's radio access, also called E-UTRAN, is expected to improve UE's throughput, 


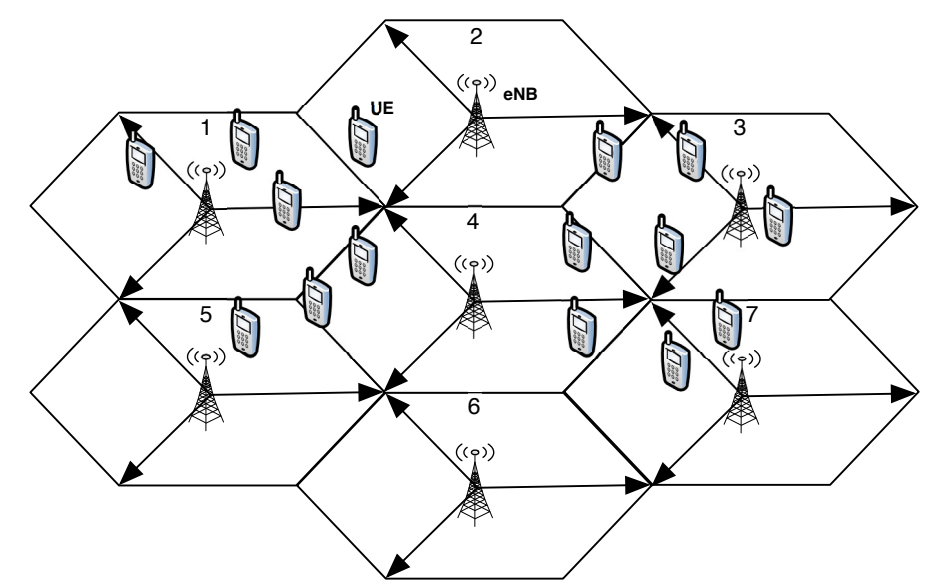

Figure 2.1: E-UTRAN network architecture

sector capacity and reduce latency, bringing substantially improved user experience with full mobility [3]. The EPC network architecture supports E-UTRAN through a reduction in the number of network elements, improved redundancy, and simpler functionality.

3GPP specified the overall system requirements for LTE networks and they are shown in Table 2.1]1]. For example, for an LTE network with $20 \mathrm{MHz}$ at $2 \mathrm{GHz}$ carrier frequency band, a maximum number of $100 \mathrm{RBs}$ is available. Also, it can provide 100 Mbps downlink and 50 Mbps uplink peak data rates, with a Macro-eNB range that can go up to $30 \mathrm{~km}$. $3 \mathrm{GPP}$ also sets high performance requirements for LTE that rely on orthogonal frequency division multiplexing (OFDM), multiple-input multiple-output (MIMO) systems and smart antennas. Another important feature of the EPC is its compatibility with other fixed line and wireless access technologies which gives service providers the ability to deliver a seamless mobility experience. 
Table 2.1: LTE requirements 1

\begin{tabular}{|c|c|}
\hline LTE Requirement & Description \\
\hline Bandwidth & $1.4,3,5,10,15$ and $20 \mathrm{MHz}$ \\
\hline No. RBs & $6,15,25,50,75,100$ \\
\hline Carrier Frequency & $700 \mathrm{MHz}, 900 \mathrm{MHz}, 1800 \mathrm{MHz}$ and $2.6 \mathrm{GHz}$ bands \\
\hline Peak data rate & $\begin{array}{c}\text { Downlink } 100 \mathrm{Mbps} \\
\text { Uplink } 50 \mathrm{Mbps} \text { at } 20 \mathrm{MHz} \text { spectrum allocation }\end{array}$ \\
\hline Coverage & $1 \mathrm{~km}-30 \mathrm{~km}$ \\
\hline Mobility & $\begin{array}{l}\text { Excellent call setup up for speeds up to } 15 \mathrm{kmph} \\
\text { Slight degradations for speeds up to } 120 \mathrm{kmph}\end{array}$ \\
\hline Duplexing & $\begin{array}{c}\text { Frequency division duplexing (FDD) } \\
\text { Time division duplexing (TDD) } \\
\text { Half-duplex FDD }\end{array}$ \\
\hline Multiple access & Downlink OFDMA, Uplink SC-FDMA \\
\hline MIMO & $\begin{array}{c}\text { Downlink } 2 \times 2,2 \times 4,4 \times 4 \\
\text { Uplink } 1 \times 2,1 \times 4\end{array}$ \\
\hline Modulation & $\begin{array}{c}\text { Quadrature phase shift keying (QPSK) } \\
\text { 16-Quadrature amplitude modulation (16-QAM) } \\
\text { 64-QAM }\end{array}$ \\
\hline Channel coding & Turbo code \\
\hline User plane latency & 5 - 15 msec user plane latency \\
\hline $\begin{array}{l}\text { Compatibility with } \\
\text { existing systems }\end{array}$ & $\begin{array}{l}\text { Works seamlessly with legacy } 2 \mathrm{G} \text { and } 3 \mathrm{G} \text { systems } \\
\text { CDMA and WiMAX networks } \\
\text { Also applies to all IP networks }\end{array}$ \\
\hline Other techniques & $\begin{array}{c}\text { Channel sensitive scheduling } \\
\text { Power control } \\
\text { Link adaptation } \\
\text { Hybrid automatic repeat request (HARQ) } \\
\text { Inter-cell interference coordination (ICIC) }\end{array}$ \\
\hline
\end{tabular}




\section{$2.2 \quad$ LTE Network Architecture}

The coverage is provided by eNBs at the centre of each site, as shown in Figure 2.1 . Each eNB is equipped with three antennas that divide each site into three sectors. The network operates with frequency re-use 1, and downlink transmissions are based on OFDM.

As explained in the previous section, the LTE network is divided into two parts: E-UTRAN and EPC, as shown in Figure 2.2. The E-UTRAN consists of UE and base stations (BS). On the other hand, the EPC consists of the elements that are needed to connect the BS to the IP core, such as mobility management entity (MME), serving gateway (S-GW), packet data network gateway (P-GW), and element management system/network management system (EMS/NMS). All these elements are explained in more details in the following subsections.

\subsubsection{Evolved Node B (eNB)}

An evolved Node B (eNB) is the base station in the E-UTRAN architecture that is responsible for communication with UE, like a BS in global system for mobile (GSM) networks. The eNB is connected to the EPC nodes by the S1 and S1-U interfaces and can also connect to its neighbor eNBs through the X2 interface. The S1 interface connects eNBs with MMEs and the S1-U interface connects eNBs with S-GWs. Below is a description of some of the functionality of eNBs:

- Cell control: eNB controls the radio resources of its own cells.

- Mobility control: eNB is responsible for controlling the mobility of active UE, which is done by ordering these UE to perform measurements and handover to neighboring eNBs when necessary.

- Shared channel handling: eNB owns the cell resources and handles the 


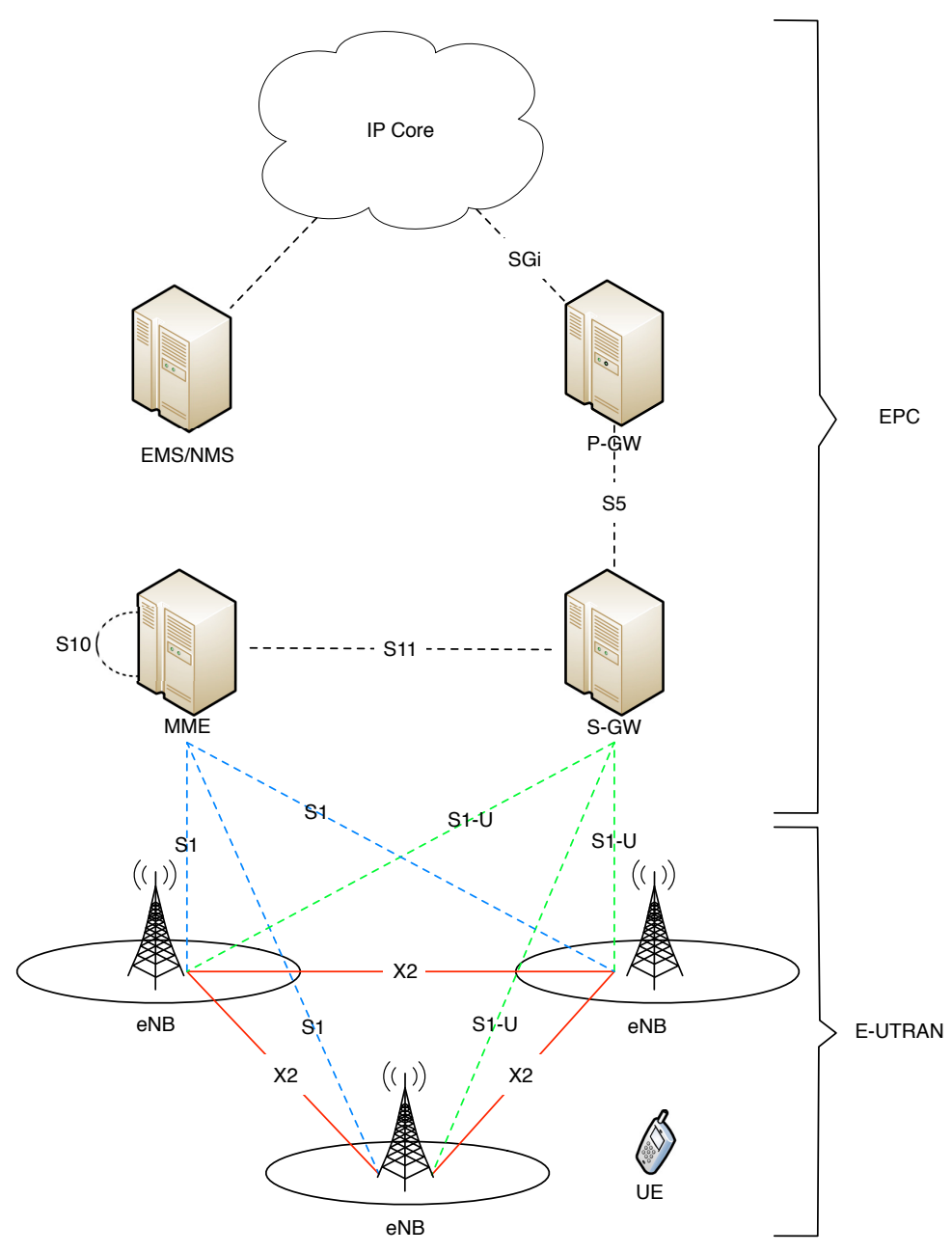

Figure 2.2: LTE architecture

shared and random access channels used for initial access and signaling.

- Scheduling: eNBs are installed with a scheduler with support for the QoS model which provides efficient scheduling of UE's data.

- Multiplexing and mapping: eNB performs mapping of logical channels onto transport channels.

- Physical layer functionality: eNB handles scrambling, beam forming, and OFDM modulation. The eNB also handles link adaptation and power control. 
- Measurements and reporting: eNB configures and makes measurements on the radio environment and eNB's internal variables and conditions that can be used for radio resource management (RRM).

- Segmentation/Concatenation: The radio link control (RLC) in the eNB supports segmentation and concatenation to adjust the payload to the transport block size.

- HARQ: A medium access control (MAC) layer with fast HARQ can provide a quick correction for most errors from the radio channel in order to achieve low delay and efficient use of radio resources for applications such as VoIP.

\subsubsection{Mobility Management Entity (MME)}

The MME is the main control node for the LTE access network. It is responsible for UE tracking and initiating paging and authentication of UE. MME retains location information for each UE and then selects the appropriate gateway. MME also allocates and generates temporary identities to UE.

MME connects to eNBs through the S1 interface and connects to S-GW through the S11 interface, as shown in Figure 2.2. Multiple MMEs can be grouped together in a pool to accommodate increased signaling load in the network. The MME is also important in handover signaling between LTE and $2 \mathrm{G} / 3 \mathrm{G}$ networks. The MME node is defined in the EPC architecture and it contains core network control functionality. The same MME controls a UE as long as it moves within the MME's pool area. The MME handles the functions listed below:

- UE attach/detach handling: Allow UE to register or de-register from the network. 
- Security - authentication, authorization and accounting (AAA): Perform ciphering and integrity protection of non-access stratum (NAS) signaling.

- EPC bearer handling: Manage the setting up, modification and tearing down of EPC bearers. An EPS bearer is a virtual connection that identifies traffic flow between UE and P-GW.

- Mobility anchor IP point of presence: Act as a mobility anchor which hides UE's mobility from the fixed network.

- Mobility management of idle mode UE: Idle mode UE is tracked by the condition of the tracking area (TA).

\subsubsection{Serving Gateway (S-GW)}

Every UE connected to the LTE network is connected to a single S-GW through the $\mathrm{S} 1-\mathrm{U}$ interface. The $\mathrm{S}-\mathrm{GW}$ is responsible for UE handovers between neighboring eNBs, also data transfer of all packets throughout the user plane. Furthermore, it is responsible of connecting to other networks such as $2 \mathrm{G} / 3 \mathrm{G}$. $\mathrm{S}-\mathrm{GW}$ is monitoring and tracking every UE during its idle state and generates paging requests when a downlink data arrives for UE, e.g. receiving a call.

\subsubsection{Packet Data Network Gateway (P-GW)}

The P-GW acts as a mobility connection between 3GPP and non-3GPP technologies. P-GW connects UE to external packet data network (PDN) by tunneling all traffic for the UE. The P-GW allocates the UE's IP address, which is used to communicate with other IP hosts in external networks. 


\subsubsection{Element Management System/Network Management System (EMS/NMS)}

The EMS provides connectivity for network elements while offering configuration and monitoring tools. The EMS maintains a list of available devices and their state, while maintaining UE security credential. On the other hand, the NMS is concerned with the network as a whole. The NMS provides access to all the functionality of an EMS, while providing a more complete view of how network elements work together. Also, the NMS simplifies the task of adding new elements to the network by reconfiguring neighboring elements.

\subsection{LTE HetNets}

In 3GPP release 12 [11], a new LTE network architecture, called HetNet, was introduced. In [12], the authors present a comprehensive overview of HetNets. This new architecture adds three new types of LTE eNBs: Micro, Pico and Femto-eNBs. In total, there are four different sizes of LTE base stations, as shown in Figure 2.3. They are as follows [13] [14:

- Macro-eNB: cell in a mobile phone network that provides radio coverage served by a high power eNB (inter-site distance $1-30 \mathrm{~km}$ ), covering a large area such as a city's downtown.

- Micro-eNB: cell in a mobile phone network served by a low power eNB (inter-site distance $\leq 250 \mathrm{~m}$ ), covering a small area such as a mall, or an airport.

- Pico-eNB: small cellular base station typically covering a limited area (inter-site distance $\leq 100 \mathrm{~m}$ ), such as indoors (offices, shopping malls, train stations, stock exchanges, etc.). 


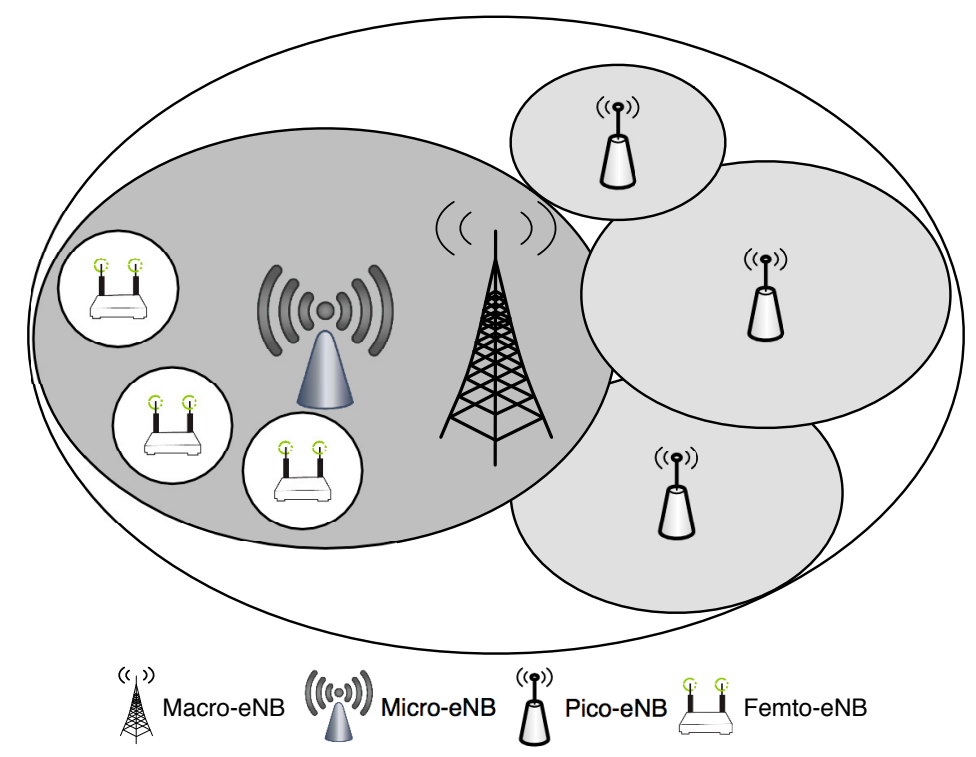

Figure 2.3: Heterogeneous networks consist of Macro, Micro, Pico, and Femto eNBs

- Femto-eNB: low-power wireless access point that operate in licensed spectrum and connected to mobile operators network using residential DSL or cable broadband connections. A Femto-eNB can usually support a maximum of 5 users in its range (inter-site distance $\leq 20 \mathrm{~m}$ ).

\subsection{LTE Power Consumption}

Electrical energy is used to describe the energy absorbed or delivered by an electrical circuit. The most common unit for electrical energy is Watt-hour (Joules). One Watt-hour of energy is equal to one Watt of electrical power maintained for one hour. While energy represents the quantity of work done, it doesnt measure how fast you can get that work done. On the other hand, power is the rate of producing or consuming energy. Which means that power is energy per unit of time and is measured in Watts. Although technically the power is not consumed, the term power consumption can be used to measure the rate of energy consumed in one hour which is equal to the 
power (Watts).

Wireless access networks are amongst the top energy consumers and a large contributor to the $\mathrm{CO}_{2}$ emissions of information and communications technology (ICT). Base stations consume the biggest part of the energy consumption, generating a large electricity bill to network providers [15]. As a result, the energy consumption of base stations is going to become an important issue in the coming years.

In [2], the power consumption of a Macro-eNB was calculated and found to be over $1800 \mathrm{~W}$. As shown in Table 2.2, over one third of that power consumption comes from air conditioning. Therefore, several solutions were proposed to reduce power consumption, such as improving eNB's hardware design, using renewable energy resources, and reducing the number of active eNBs [16]. Switching off unnecessary devices and wireless resources has become the most popular method to reduce power consumption in cellular networks. The power consumption grows proportionally with the number of cells.

Since power consumption is not proportional to an eNB's load level, an idle eNB will still consume a significant amount of power. Consequently, switching off one or more antennas will not have a big effect on power consumption . This is mainly because power amplifiers and air conditioning are the main components of power consumption . Therefore, algorithms should be developed to switch off an entire eNB rather than just one or two antennas [17].

Figure 2.4 shows a simplified diagram of a complete eNB, which can be generalized to all types of eNBs. An eNB generally comprises multiple transceivers with multiple antennas. The percentage of power consumption by different Pico-eNB transceiver components is shown in Figure 2.5. A transceiver is made of antenna interface, power amplifier, radio frequency, baseband unit (digital signal processor), main supply, cooling system and power supply.

The power consumption for each part of the eNB is a constant value, except for 
Table 2.2: Power consumption of different parts of Macro-eNB [2]

\begin{tabular}{lc}
\hline Equipment & Power consumption \\
\hline Digital signal processing & $100 \mathrm{~W}$ \\
Transceiver & $100 \mathrm{~W}$ \\
Signal generator & $384 \mathrm{~W}$ \\
AC-DC converter & $100 \mathrm{~W}$ \\
Air conditioning & $690 \mathrm{~W}$ \\
Microwave link & $80 \mathrm{~W}$ \\
\hline & $350 \mathrm{~W}$ \\
Power Amplifier SISO (1x1) & $6.3 \%$ \\
& $43 \mathrm{dBm}$ \\
\hline
\end{tabular}

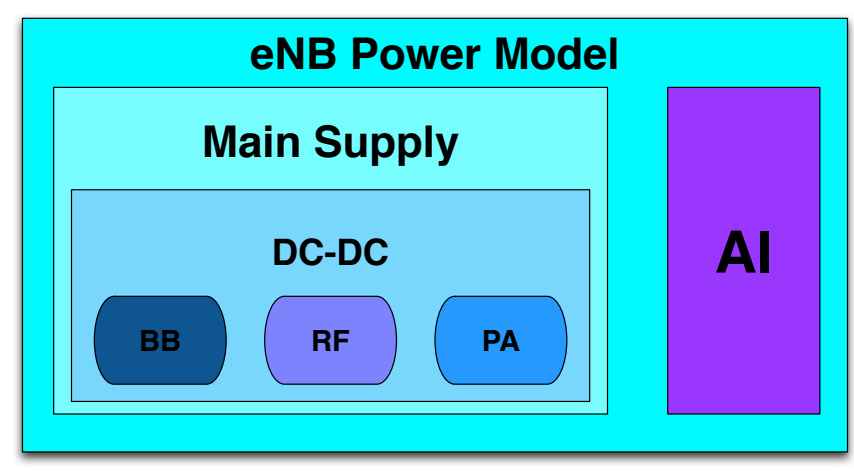

Figure 2.4: Simplified block diagram of an eNB.

the power amplifier $P_{a m p}$, whose power consumption depends on the transmit power of the antenna $P_{t x}$ and can be calculated with Equation 2.1. In this equation, $\eta_{a m p}$ is the efficiency of the power amplifier, which is the ratio between the output and the 


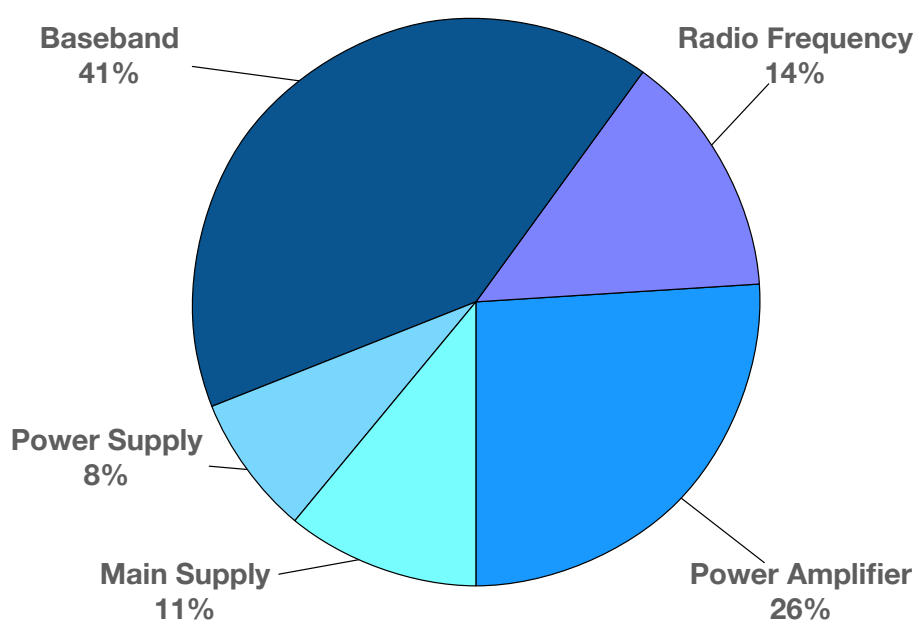

Figure 2.5: Pico-eNB power consumption breakdown for different components

input power of the amplifier.

$$
P_{a m p}=\frac{P_{t x}}{\eta_{a m p}}
$$

The required electrical power $\left(P_{e l}\right)$ for the entire eNB can be calculated with Equation 2.2, where $n_{\text {sect }}$ and $n_{t x}$ are the number of sectors in a cell and the number of transmitting antennas per sector respectively. The $P_{B B}, P_{R F}, P_{M S}, P_{P S}$ are required power for the baseband, radio frequency, main supply and power supply components of the eNB respectively. The $P_{A C}$ is the required power for the air conditioner that is available in Macro-eNBs only.

$$
P_{e l}=n_{\text {sect }} \cdot\left(n_{t x} \cdot P_{a m p}+P_{B B}+P_{R F}+P_{M S}+P_{P S}\right)+P_{A C}
$$

The power consumption of all three types of eNBs discussed earlier is shown in Table 2.3. Because Macro-eNBs consume $1800 \mathrm{~W}$ compared to $35 \mathrm{~W}$ for Pico-eNBs, in this thesis, we propose to replace each Macro-eNB with a high number of PicoeNBs to cover the same area. Moreover, by turning some of these off when they are not needed, the gain can be even more significant. 
Table 2.3: Power consumption of different types of eNBs 2

\begin{tabular}{lccc}
\hline Type of eNB & $\begin{array}{c}\text { Power } \\
\text { Consumption } \\
(\mathbf{W})\end{array}$ & $\begin{array}{c}\text { Number of } \\
\text { Active UE }\end{array}$ & $\begin{array}{c}\text { Required Power } \\
\text { per UE (W) }\end{array}$ \\
\hline Macro & 1800 & 2000 & 0.9 \\
Micro & 130 & 512 & 0.25 \\
Pico & 35 & 192 & 0.18 \\
\hline
\end{tabular}

\subsection{Introduction to $\mathrm{SON}$}

Currently, network operators monitor network quality, including fault, configuration, accounting, performance, security (FCAPS), which requires high human workload to achieve the desired QoS [18].

The implementation of LTE means additional operation challenges and network integration. LTE runs in parallel with existing mobile networks. The complexity of the network increases with the large number of base stations and the different types of eNBs that are used. Network optimization involves base station maintenance, testing, signaling, and data collection to improve coverage and reduce interference [6]. In order to reduce operational cost and effort, the concept of self organizing networks (SON) is being introduced in LTE. SON allows automatic and dynamic adaptation to varying situations of the network without human intervention [19].

\subsubsection{SON Functions}

The concept of SON is built around three different functions as described below. 


\subsubsection{Self-Configuration}

Self-configuration deals with all tasks necessary at the pre-operational phase of network deployment, such as planning and initial configuration. The aim of selfconfiguration is to accelerate the integration of new base stations into a network [20]. The self-configuration process consists of the following steps [19]:

1. The eNB is delivered with initial basic software to allow the basic deployment process and software for the basic connectivity.

2. An IP address is allocated to the eNB by the dynamic host configuration protocol (DHCP) server and a connection to the transport network is established.

3. A secure IP connection is established between the eNB and authentication centre.

4. Configuration data and software are downloaded from the operation and maintenance $(\mathrm{O} \& \mathrm{M})$ subsystem.

5. The eNB establishes S1 connection to the core network.

6. The eNB establishes X2 connections to neighbor eNBs.

\subsubsection{Self-Optimization}

Self-optimization is used to improve network quality by optimizing network parameters on the fly. Optimization is important to ensure that once an eNB has been installed, it operates efficiently. Self-optimization techniques can analyze the performance of the network and change its operation so that it provides the best QoS. The main tasks involve handovers and balancing loads between neighboring sites [21]. 


\subsubsection{Self-Healing}

Today's cellular networks are extremely complicated and failures occur from time to time. Another important function of SON is to enable the network to self-heal. The self-healing function enables failure detection using cell degradation function and masks its effect to users while repairs are undergone on the cellular network. It can do this by changing the boundaries of adjacent cells to accommodate the outage using cell outage compensation (COC) function [22].

\subsubsection{SON Architecture}

Three different SON architectures are briefly described below along with their main advantages and disadvantages [19].

\subsubsection{Centralized SON}

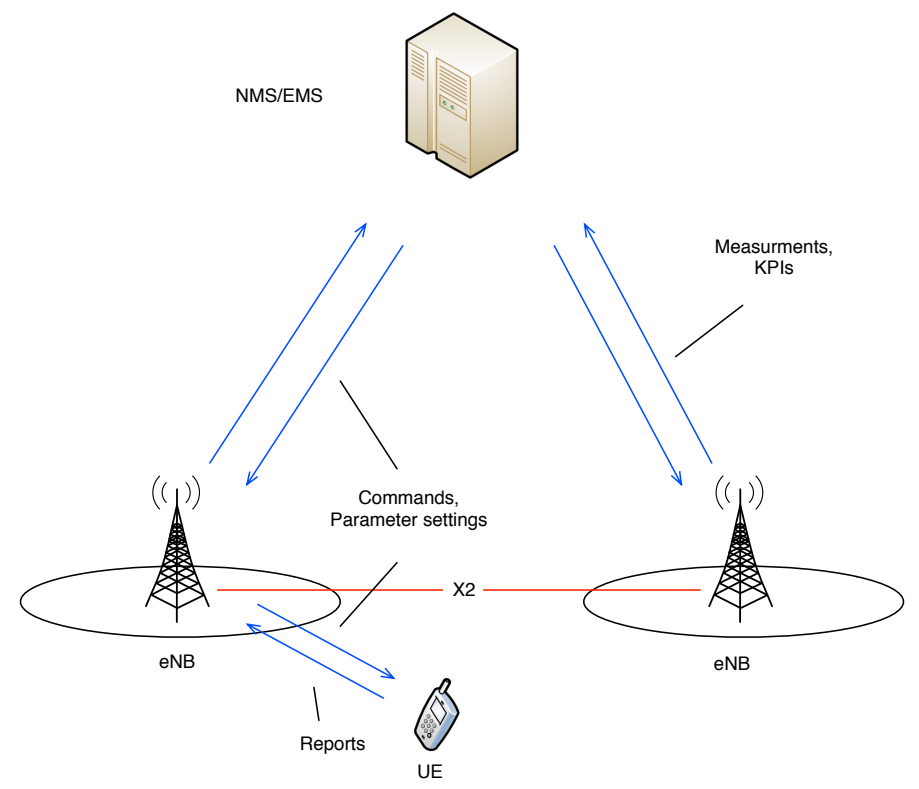

Figure 2.6: Centralized SON architecture

In a centralized SON architecture, the algorithms are executed in the EMS/NMS. 
Commands, requests and parameter settings data flow from the EMS/NMS to the eNBs while measurement data and reports flow in the opposite direction, as shown in Figure 2.6.

A main advantage of the centralized approach is that the SON algorithms can take information from all eNBs into consideration. This means that it is possible to optimize parameters of all centralized SON functions to globally optimize the network. Also, SON functions can be coordinated easily because the control of all SON functions is done centrally. Another advantage is that third party SON solutions are possible, since functionality can be added at the EMS/NMS and not in each individual eNB.

The main disadvantages of the centralized SON architecture are:

- Longer response time, which can limit how fast the network can adapt to changes and can cause network instabilities.

- Increased backhaul traffic, because measurement data has to be sent from the eNBs to the EMS/NMS and instructions must be sent in the opposite direction. As more cells are added to the network, the traffic becomes very significant.

- It represents a single point of failure.

\subsubsection{Distributed SON}

In a distributed SON architecture, the SON algorithms are run in the eNBs and the eNBs exchange SON related information directly with each other, as shown in Figure 2.7. This architecture allows to push its functionality to the far edges of the network even when the number of eNBs increases. With this approach, each eNB is equipped with smart and intelligent SON algorithms which can reduce complexity, load and footprint issues with EMS/NMS.

The main disadvantages of this architecture are: 


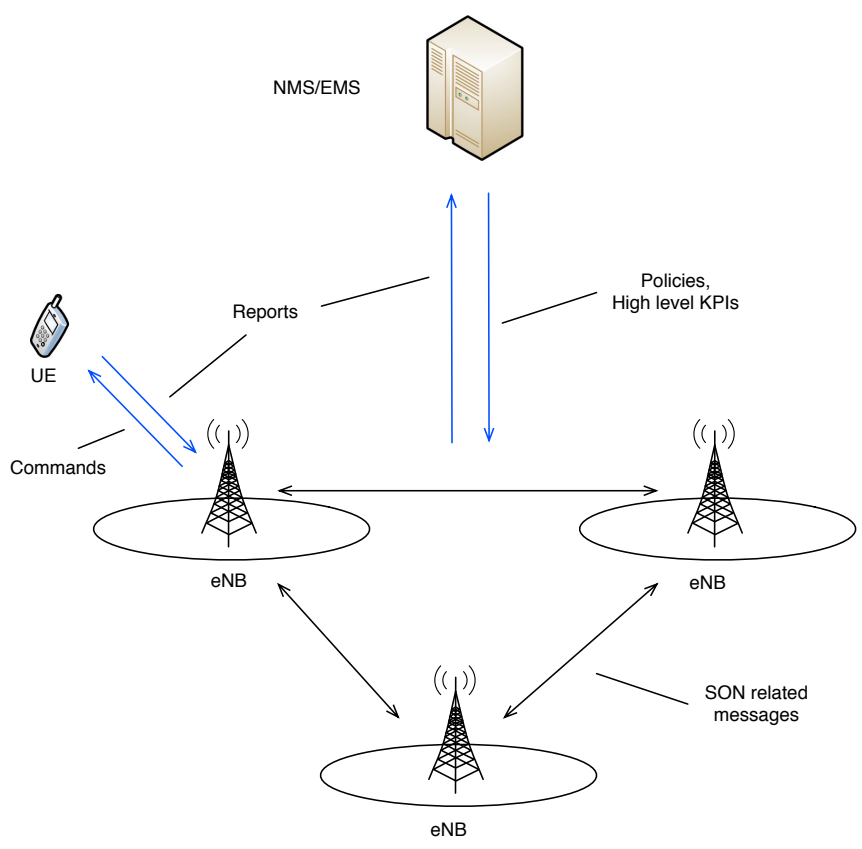

Figure 2.7: Distributed SON architecture

- The sum of all the optimizations done in eNBs do not necessarily result in optimum operation for the whole network.

- The implementation of the SON algorithms in eNBs are vendor specific, so third party solutions are difficult to integrate.

Even if the algorithms themselves are executed in eNBs, the EMS/NMS is usually able to control the behavior of the SON function.

\subsubsection{Hybrid SON}

The hybrid architecture is a mix between distributed and centralized architecture. In Hybrid SON, algorithms are executed at two or more of the following levels: eNBs or EMS/NMS. Unfortunately, the disadvantages of both centralized and distributed SON are also inherited. 


\section{Chapter 3}

\section{Literature Review}

In this chapter, we summarize the related work published in the literature. We first review three types of energy saving approaches for LTE networks: dynamic, static, and hybrid switch on/off algorithms. Then, we discuss eNB switch on/off algorithms related to different types of heterogeneous networks. Finally, we summarize the literature review and provide the significance of this thesis over the mentioned papers.

\subsection{Introduction}

Wireless service providers are pursuing many options to address the growing demand for mobile broadband services. The need for higher bandwidths has increased because of the popularity of smart-phones and their applications that require high data rates. In order to accommodate that rise in demand, more eNBs have to be installed which results in an increase of the network's complexity, and thus making the SON feature in LTE even more attractive [23]. Also, the rapidly growing number of eNBs has contributed to the increase of power consumption of cellular networks. As operators install more eNBs to fulfill the need for higher capacity, the power consumption of cellular networks increases. Increasing the number of eNBs in the network can be seen as bringing the network closer to the users [16 [17]. On the other hand, several 
eNBs become underutilized outside peak traffic time and consume almost full power as explained in the previous chapter.

The implementation of LTE means additional operation challenges and network integration. The complexity of the network increases because of the large number of HetNets and base stations. Currently, network operators monitor network performance, including FCAPS, all of which require a high human workload to achieve the desired QoS [18]. Network optimization involves base station maintenance, testing, signaling, and data collection to improve coverage and reduce interference [6]. In order to reduce operational cost, effort and human errors, SON is being introduced in LTE. SON allows for automatic and dynamic adaptation to varying situations of the network without human intervention [19].

\subsection{Energy Saving Approach for LTE networks}

The excessive energy consumption of a large number of eNBs in the network conflicts with the energy efficiency requirements of LTE networks [16]. Therefore, eNB switch on/off was proposed in 24 to save energy by switching off or changing to sleep mode underutilized eNBs and handoff their users to neighboring eNBs while maintaining an acceptable QoS.

Implementing switch on/off algorithms is a complicated problem, in particular since switching off the wrong eNB at the wrong time may worsen the overall system performance. Strategically switching on/off eNBs based on UE traffic is an important way to reduce energy consumption in LTE networks. There are two main approaches for switching on/off eNBs: dynamic and static. Dynamic switch on/off approaches are based on immediate UE demand and network state, which makes them more complicated and difficult to model. On the other hand, static switch on/off approaches are based on a set of different combinations that are stored in look-up tables. As a result, 
static switch on/off approaches are more tractable and realistic for energy saving. The static switch on/off algorithms become more complex as the number of eNBs in the network increases, because they can not examine all possible combinations.

\subsubsection{Dynamic Energy Saving Approach}

The dynamic switch on/off algorithm is executed in real time to determine which eNBs to switch on/off, which requires a global knowledge of the network state including the channel state between UE and eNB, and the load level of all eNBs in the network. Because of the vast size of this information, it is difficult to be exchanged by the network in a timely manner. Furthermore, for larger sized networks, the optimization's computational time will be constraining [25].

Several dynamic switch on/off algorithms were proposed in [26] [24] and [27], which rely on heuristic methods. Heuristics are a reasonable method for such complicated scenarios because they provide good solutions in a timely manner.

Researchers in 28] considered tackling the switch on/off approach using a utilitybased algorithm. In that research, they tested two network setups with 4 and 9 Macro-eNBs, and 10 or 20 UE. They also assumed that each UE requires a fixed data rate of $500 \mathrm{kbps}$ regardless of the network quality, which means that as the number of UE connected to an eNB increases, the power consumption also increases to be able to provide the required data rate.

In [27], centralized and decentralized switch on/off algorithms for Micro-eNB homogeneous networks (HoNets) were suggested. They proposed centralized and decentralized energy saving approaches. In the centralized algorithm, each BS's traffic load is examined to determine if the BS will be switched on/off. On the other hand, in the decentralized algorithm the BS's traffic load is estimated locally and the algorithm decides if it is switched on/off. Furthermore, they used two energy modes for each Micro-eNB: active and sleep/standby. For the latter mode, it was assumed 
that Micro-eNBs consume negligible energy, which is not the case as explained in Section 2.4. Also, they did not take into account the interference caused by surrounding eNBs in calculating the spectral efficiency of the network which made the result unrealistic.

A dynamic distributed switch on/off algorithm for HoNets was proposed in 29]. They introduced network-impact factor for each Macro-eNB, which is how much additional load switching off a Macro-eNB will bring to the neighboring eNBs. They also explained in details the process of switching on/off an eNB after the algorithm decision is made. Although the algorithm is considered a dynamic algorithm, the authors did not take into consideration the time needed for the eNB to switch from off to on mode. The latter is an important factor as by the time the eNB is switched on, the UE information has changed and the algorithm's decision might be invalid at this point.

A distance aware dynamic switch on/off algorithm was proposed in [30]. Each eNB calculates the distance to its associated UE and to the UE associated to its neighboring eNBs. Then, all eNBs are sorted according to the total average distance calculated in the previous step. The algorithm then switches off the eNB with the maximum average distance, because greater distances lead to higher transmission power (higher power consumption).

In [31], a traffic-and-interference aware switching strategy for BS and relay station (BS-RS) switching patterns was presented. In a BS-RS switching model, a BS changes into an RS during off-peak traffic instead of switching off. The study in 32 devises a sleep mode mechanism in Macro-eNB HoNets that maximizes the energy savings of the network while maintaining both the user reliability and the throughput performance of the network.

Researchers in [33] investigated the transition time for switching off one predetermined BS and handing over UE to a new BS. They found that the average transition 
duration was of the order of 1 minute at most. In that study, they did not define an algorithm to decide which BS to switch on/off.

A simple greedy-drop algorithm was introduced in [24], where BSs are switched off starting with the least loaded one. The algorithm terminates when it finds the first BS that cannot be switched off because not all its users can be handed over to neighboring BSs. The same researchers also proposed a greedy-add algorithm to switch on as few BSs as possible. BSs were switched on based on traffic demand and the algorithm terminates when all users are connected to a BS. In that research, Micro-eNBs were used with two energy mode (active and sleep mode). The results show that the proposed algorithm can support the trade-off between outage probability and energy consumption. The drawbacks of the proposed algorithms are:

- When a Micro-eNB is in sleep mode, it consumes $10 \mathrm{~W}$ which can add up when the number of eNBs increases.

- Constant data rate was required for each UE (122 kbps), which does not portray a realistic test scenario.

Authors in [34 presented a novel optimization model that can be used for energysaving at the UMTS cellular access network level. They also explored the influence of BSs switching on/off on the reduction of network energy consumption in the time domain. They assumed a fixed data rate for their UE. They also neglected that the network adapts to the data rate of each UE based on the channel quality.

A dynamic BS sleeping algorithm based on blocking probabilities was proposed in 35]. The BSs switch between active and sleeping modes according to the traffic load variations with respect to the blocking probability constraint. 


\subsubsection{Static Energy Saving Approaches}

Interference modeling is an important and challenging issue in switch on/off algorithms, because it is hard to know the set of active eNBs beforehand. Usually, UE are connected to the eNB that provides the best downlink signal-to-interference-plusnoise ratio (SINR). Nonetheless, when an eNB is switched off, UE connected to it need to be handed over to another neighboring eNB. Lacking a proper interference characterization, these UE might experience a large amount of interference because the set of active eNBs that contributes to the interference is known only at the final stage. Therefore, the anticipated network performance is inaccurate. Certainly, some switch on/off algorithms assume zero interference, which means that there is perfect inter-cell interference coordination (ICIC) [24]. However, this assumption is too optimistic and can produce an impossible upper bound on the number of switched off eNBs. On the other hand, some algorithms assume that all eNBs contribute to the interference as if they were active at all times [26], which can result in a poor lower bound on the number of switched off eNBs.

One way to reduce the effect of the interference problem is to predetermine the set of eNBs that actually generate the interference in the network. In static switch on/off algorithms, different combinations of active eNBs are predetermined offline for different traffic densities, following which network operators choose which combination accommodates the current traffic density. Static switch on/off algorithms are usually appropriate for long time scale, such as hours, and generally are based on historical traffic distribution [36] 37].

Static switch on/off algorithms are different from dynamic switch on/off algorithms because the network configuration remains the same for a long period of time. Accordingly, an appropriate interference modeling can be achieved by considering only the predetermined combination of active eNBs. Furthermore, static switch on/off 
algorithms can be viewed as a cell planning problem with a constrained combination of eNB locations. In cell planning, the eNB placement is based on larger set of possible locations while in static switch on/off algorithms, the possible locations are restricted to actual deployed eNBs.

In [38], a multi-objective framework that takes into account the traffic behavior in Micro-eNB network switch on/off decision making was proposed. In that research, all UE require a fixed data rate of $250 \mathrm{kbps}$ and they are stored and served based on their spectral efficiency. This means that UE with lower spectral efficiency might not be served if there is not enough bandwidth.

Researchers in 39 proposed flexible schedulers for both uplink and downlink that allow coverage extension in Macro-eNB HoNets in urban and rural environments. They also proposed several base station switching patterns (1 or 2 out of 3 MacroeNBs on and 3 out of 4 Macro-eNBs on) during low traffic periods (1 to 5 users per $\mathrm{km}^{2}$ in rural areas and 4 to 68 users per $\mathrm{km}^{2}$ in urban areas). The study did not address the problem of deciding when to change from one pattern to another, which means that they cannot follow the change in UE locations.

A special case of static switch on/off algorithms is known as switch on/off patterns. The set of active eNBs is predetermined offline and is selected relative to spatial pattern and resembling known frequency reuse patterns [36 [8]. Using switch on/off patterns minimizes the coverage holes caused by switching off eNBs. Moreover, switch on/off patterns are more energy efficient in the uplink because there is always an active eNB nearby [40]. In [40], researchers suggested a switch on/off algorithm that considers the UE power consumption as a performance indicator in the decision of finding which eNB to switch on/off in HoNets. Researchers in [8] investigated the effect of different switch on/off patterns on the outage probability. The switch on/off patterns used in that research does not incorporate the interference in calculating the channel state information. 
Authors in [41] proposed a switch-off decision based on the average distance between eNBs and UE, rather than the eNB's traffic load. The algorithm proposes to switch off the eNB that has the maximum average distance. In their simulation, the algorithm was evaluated for low traffic network with an average number of $10 \mathrm{UE}$ per eNB, and the results concentrated mainly on the energy reduction without taking into account the degradation of the network data rate when they switch off eNBs.

In [42], the authors studied the effect of cell zooming in conjunction with switching off eNBs on the energy efficiency of cellular networks. They proposed an evaluation method using spatial patterns to determine which eNB to switch off, while the remaining active eNBs extend their coverage area by increasing their transmit power. They also suggested increasing the power of all eNBs regardless if it was neighboring a switched off eNB or not, which can lead to an increase in the ICI and in return a decrease in the SINR of the network.

Throughput and energy consumption of coordinated and uncoordinated scheduled sleep modes with orthogonal or partially orthogonal transmission periods were proposed in [43]. The sleep periods were scheduled over the LTE radio frame and the sleep period has the same fixed duration in each cell. For example, each sleep period has a duration of 7 subframes and for each subframe, only one eNB can be active. The main drawback of the proposed algorithm is that the sleep period duration is very short ( 7 subframes $=7 \mathrm{msec})$. The amount of energy consumed by the sleeping Macro-eNBs will be more evident with the increase in the number of Macro-eNBs in the network, which in turn will increase the complexity of the switch on/off algorithm.

Researchers in [44] investigated different cell sorting criteria for Micro-eNB HoNets. The simulation results showed that, by sorting eNBs based on the number of UE that can be served, more energy can be saved compared with sorting eNBs based on their current load. In that simulation, they used a fixed data rate (500 kbps) 
and they assumed that the spectral efficiency was calculated based on the SNR between the UE and the Micro-eNB. That assumption makes the problem tractable, but also, unrealistic.

Different approaches for switching off a specific number of BSs during low traffic periods in UMTS cellular networks are presented in [45], [46] and [47]. The energy reduction resulting from switching off a randomly chosen number of BSs is computed by simulating UMTS cellular networks in [45]. The same authors presented in [46] an improvement of their former work as they proposed a hierarchical and uniform dynamic network planning for switching BSs on and off. Researchers in [47] used a deterministic traffic variation pattern to optimize energy savings by assuming that any fraction of cells can be switched off over time. In these studies, the switching off pattern is fixed and the traffic is assumed to be uniformly distributed over the whole network.

\subsubsection{Hybrid Energy Saving Approaches}

The hybrid switch on/off algorithms are a mix between dynamic and static switch on/off algorithms, meaning that a dynamic switch on/off algorithm needs to be executed on top of the static one. In hybrid algorithms, a group of active eNBs, which are controlled by a static algorithm, are chosen to provide coverage and collect network information, while the remaining eNBs are controlled with a dynamic switch on/off algorithm to accommodate the fast change in user demand. Hybrid switch on/off algorithms are especially useful when UE are non-uniformly distributed in the network. Unfortunately, the disadvantages of both dynamic and static switch on/off algorithms are also inherited.

In [37], researchers investigated the effect of dynamic, static and hybrid switch on/off algorithms on the outage probability and the network power consumption in Macro-Micro-eNB HetNets. The three switch on/off algorithms were tested for low 
traffic UE in the network, i.e. voice services with a rate of $12.2 \mathrm{kbps}$.

It is important to remark that in the previous works regarding energy saving techniques, the decision to switch off a BS or transfer it into sleep mode was based on either fixed traffic or on the power consumption of the network. However, because of the increase in the expected data rate and greening of LTE networks, such algorithms will not be sufficient to provide that.

\subsection{Heterogeneous Network Deployment}

The increase in demand for mobile data and development of new mobile applications will continue to drive data traffic growth in an exponential fashion. This forces network operators to review their network design and increase the capacity of their networks. Researchers in 48 focused on providing an overview of Macro-Pico-eNB HetNet network design. They also provided a theoretical analysis and a simulated performance analysis of a Macro-Pico-eNB HetNet, which was evaluated according to the total sector throughput, the fraction of UE in the network connected to Pico-eNBs and the average throughput per UE connected to a Macro-eNB or a Pico-eNB.

In [49], researchers investigated four sleep modes for small eNBs (Micro and PicoeNB) in multi tier HetNets. These sleep modes were applied only to the small eNB tier, while Macro-eNBs are assumed to be fully operational all the time. The power consumption of the Macro-eNB can be a drawback of the proposed algorithm. By switching off both small and large eNBs, further power reduction is possible. Simultaneous sleep mode optimization for multiple tiers is still an open issue.

In the following sections, related work on the energy efficiency of downlink two-tier HetNets is discussed. 


\subsubsection{Macro-Pico-eNB Deployment}

In [50], the HetNets deployment strategy was investigated by overlaying Pico-eNBs within the Macro-eNB network, and its impact on energy consumption was determined. The authors considered the percentage of gain in terms of the served eNB throughput as a performance metric and showed that a $30 \%$ gain can be achieved for full-load scenarios.

In [51], a joint deployment of Macro-eNBs and publicly accessible user-deployed residential Pico-eNBs show that it can reduce the total network energy consumption in urban areas with high data rate user demand. Researchers in [52 also investigated the energy efficiency in the downlink of a network of Macro-eNBs overlaid by randomly distributed Pico-eNBs. They evaluated the two-tier HetNet performance in terms of resource allocation fairness and energy efficiency. In [53], researchers evaluated the energy efficiency of Pico-eNBs and LTE-advanced (LTE-A) relay deployments. The results showed that Pico-eNBs and relay nodes achieve a significant gain in power consumption in the uplink and downlink, whereas relay nodes and MacroeNB deployments provide similar power consumption in the downlink. The power reduction in these three papers is due to the Macro-eNB offloading some of its UE traffic to the Pico-eNBs, which leads to a reduction in the power consumption of the Macro-eNB. The amount of energy reduced from the Macro-eNBs is greater than the Pico-eNB's low power consumption. Such concept calls for a switching on/off algorithm to push the greening of the network even further.

The authors in [54] and [55] discussed three different sleep mode strategies for Pico-eNBs in Macro-Pico-eNB HetNets. These strategies can be controlled by the Pico-eNB itself, core network or UE. The results show that by offloading the traffic from Macro-eNBs to Pico-eNBs, the network can be redesigned and a lower number of Macro-eNBs are needed, which leads to further energy reduction. The main factor 
that was used to evaluate the network performance was the energy consumption of the network, as the required traffic for all UE is fixed to $500 \mathrm{kbps}$. Also, the proposed sleep modes were only for Pico-eNBs, which means if similar sleep modes were applied to Macro-eNBs as well, then the energy saving of the network could be increased.

In [56], the authors proposed a distributed BS on/off control mechanism for improving the energy efficiency of a Macro-Pico-eNB system. Pico-eNBs can be activated by a neighboring Pico-eNB or Macro-eNB, and deactivated by the Pico-eNB itself. The results show a reduction in the power consumption of the network as compared to a traditional control mechanism. A Pico-eNB can go to sleep mode only if all its UE can be handed over to neighboring eNBs and provided minimum data rate. This can lead to a degradation in the performance of the network as deactivating a Pico-eNB may reduce the data rate of the UE.

Researchers in [57] considered a HetNet with Pico-eNBs in a hot spot area within a Macro-eNB area. The transmit power of Macro-eNBs is much higher than the Pico-eNBs', meaning that switching off some Pico-eNBs causes many UE in these hot spots to connect to the Macro-eNB. Therefore, they proposed deploying a Pico-eNB that cannot be switched off at all times at the centre of the Pico-eNB cluster, insuring that all UE in hot spots connect to Pico-eNBs rather than Macro-eNBs when a few Pico-eNBs are switched off. Furthermore, they proposed three energy saving PicoeNB modes: on, off and lower work modes. The algorithm switches Pico-eNBs to one of these modes in order to minimize both the power consumption and the outage probability. In their simulation, they assumed all UE use the same fixed data rate of 100 kbps. The proposed algorithm focused on switching on/off only Pico-eNBs in hot spots while keeping all Macro-eNBs on all the time, meaning that these Macro-eNBs are consuming energy even if they are not serving any UE.

The authors in [58 proposed energy saving strategies for LTE Macro-eNB HoNets 
and Macro-Pico-eNB HetNets. They also investigated the impact of strategic and random sleeping on energy efficiency and power consumption. Numerical results showed that the gain in energy efficiency depends on the type of sleeping strategy used. The decision in those algorithms was based on the energy consumption and the outage probability of the network, without taking into consideration the possible degradation in network throughput caused by switching off Macro-eNBs. Furthermore, the proposed sleep modes were applied only for Macro-eNBs in HetNet networks, which keeps more energy to be reduced by switching off both Macro and Pico-eNBs in the network.

In [59], the authors proposed installing two Pico-eNBs for every Macro-eNB at the appropriate locations and allocating separate resources to all the Pico-eNBs on a global level. As a result, a $45 \%$ gain in the performance of the network can be achieved. They also suggested that switching off Macro-eNBs at night can reduce the operation cost significantly. Switching off Macro-eNBs as suggested while replacing them with 2 Pico-eNBs for each Macro-eNB can lead into coverage holes, as the coverage area a Macro-eNB is much larger compared to the Pico-eNBs. For the proposed idea to be feasible, a larger number of Pico-eNBs needs to be installed to cover the Macro-eNB area that they are replacing.

Researches in [60] proposed two centralized algorithms to dynamically switch on/off Pico-eNBs in a Macro-Pico-eNB HetNets. The aim of both algorithms is to reduce the energy consumption of the network while maintaining the QoS, which is given in terms of required service rate. They used blocking probability as a way to measure the performance of both switch on/off algorithms. They considered the transmit power as the power consumption of eNBs in the network, which represents the energy of the downlink channel not the power consumption of the network. Although they assumed a dynamic switch on/off algorithm, which means that the switching on/off of Pico-eNBs should be done simultaneously with traffic variation, 
they ignored the execution time of the algorithm in their simulations, which makes their results unfeasible.

The approach discussed in [61] reduces the energy-per-information-per-bit by making Pico-eNBs in Macro-Pico-eNB HetNets transmit with full load during good channel conditions to support a high data rate and go into sleep mode otherwise. The drawback of this algorithm is that not transmitting during periods of bad channel conditions will increase the packet delay, which reduces the QoS of the network. Furthermore, the algorithm was evaluated for a network of 1 Macro-eNB and1 Pico-eNB with only $3 \mathrm{UE}$, which does not give sufficient results to support their claim.

\subsubsection{Macro-Micro-eNB Deployment}

Reference [62] examined the impact of the deployment of random Micro-eNBs with varying density on the energy efficiency of cellular networks. The Macro-eNB transmit power was reduced to fulfill the coverage condition of $95 \%$, which led to a $20 \%$ energy efficiency gain. The Macro-eNB was used to serve UE in the outage areas between Micro-eNBs. The authors in [63] and [64] investigated the impact of various deployment strategies of HoNets, pure Macro-eNBs and Micro-eNBs, in addition to HetNets with a varying number of Micro-eNBs within the conventional MacroeNBs based on energy efficiency and traffic load conditions. The results of the study showed that Micro-eNB HoNets offer a superior energy efficiency compared to HetNets. Results also show that the best way to improve the energy efficiency when network traffic load increases is by deploying more micro sites. The same authors provided in [65], a framework to evaluate and optimize cellular network deployment with respect to the average number of Micro-eNBs per Macro-eNB and to MacroeNB size. The authors introduced the concept of area power consumption, which is the average power consumed in a cell divided by the average cell area measured in Watts $/ \mathrm{km}^{2}$. The simulation results showed that deployment of Micro-Macro-eNB 
HetNets allows a significant decrease in the area power consumption in the network while maintaining area throughput targets. The network efficiency of the proposed Macro-Micro-eNB networks (in papers [62], [63], [64] and [65]) can be improved by switching off Micro-eNBs during low traffic times and shifting the load to other eNBs.

The energy efficiency of Macro-eNB HoNets and Macro-Micro-eNB HetNets in LTE-A has been investigated in [66] by reducing the number of active Macro-eNBs and implementing a sleep mode based on the overall energy consumption and following network traffic load conditions.

\subsubsection{Macro-Femto-eNB Deployment}

A recent study [67] presented an analytical model of power consumption in MacroeNB, Micro-eNB, Pico-eNB and Femto-eNB based networks. This paper discussed different network setups, depending on UE density, traffic and required coverage, including only Femto-eNBs, Macro-Femto-eNB HetNet, Femto-Pico-Micro-eNB HetNet and Femto-Pico-Micro-Macro-eNB HetNet. All simulation results are compared to a Macro-eNB HoNet. Simulation results showed that using only Femto-eNB HoNet can reduce power consumption by $82.72-88.37 \%$. Results also showed that using Macro-Femto-eNB HetNet can achieve a 78.53-80.19\% reduction in total transmitted power by the BSs. Simulation also showed that Femto-Pico-Micro-eNB HetNets can reduce total power consumption by 9.19 - 9.79\%. Lastly, simulation results demonstrated that using Femto-Pico-Micro-Macro-eNB HetNets can achieve a 5.52 - 5.98\% reduction in total transmitted power.

In [68], the impact of Femto-eNB deployment on the capacity of Macro-eNB HoNet has been studied. Capacity models for Macro-Femto-eNB HetNets were developed taking into account traffic arrivals and departures after a finite service duration. Results showed an increase in energy efficiency when deploying Femto-eNBs within Macro-eNB HoNets. The same authors, in [69], further studied the optimal 
sleep/wake up schemes for Femto-eNB deployed in Femto-Macro-eNB HetNets to offload part of the Macro-eNB traffic. Their goal is to minimize the energy consumption of the overall HetNet while maintaining the UE QoS.

A recent research [70] focused on the main challenge of ICIC of Femto-Macro-eNB HetNets. It proposed an enhanced ICIC mechanism to suppress such interference by blanking two OFDM symbols. They also discussed a novel model to repetitively design physical downlink control channel (PDCCH) scheme to avoid ICIC effectively. In this paper, researchers did not consider the reduction in the power consumption of the network achieved by overlaying Femto-eNBs within the Macro-eNB network.

Researchers in [71], studied the distribution of downlink SINR in a Macro-FemtoeNB HetNet. They derived a general expression for the SINR at the UE from the serving eNB depending on what kind of eNBs are allowed to serve the UE. They computed the probability that a UE can connect to either a Macro-eNB or FemtoeNB. Given a Macro-eNB and Femto-eNB densities, this probability was found to be a function of two parameters:

- The minimum distance between the UE and the nearest Macro-eNB.

- The transmit power, density and link geometry of the Femto-eNBs compared to the Macro-eNBs.

In [72], an energy-efficient cell breathing and offloading mechanism in MacroFemto-eNB HetNets was studied. They suggested reducing the number of MacroeNBs on by offloading their traffic to Femto-eNBs. Also, they considered separated spectrum bands for the Macro-eNBs and Femto-eNBs, which does not include the intracell interference in the decision making of this algorithm. Furthermore, this technique is suitable only for traffic with low mobility users, which makes it not applicable in high speed mobility zones. 
Researchers in $[73]$ proposed four sleep modes for Femto-eNBs in Femto-MacroeNB HetNets. They concentrated mainly on reducing the power consumption of the sleep modes while increasing the speed of taking a Femto-eNB out of sleep mode in order to maximize the energy saving. In that research, they used signal to noise ratio (SNR) to calculate the network data rate which does not include the interference caused by different eNBs in the network. Therefore, the results are not accurate and do not represent a real life scenario.

The above mentioned papers focused mainly on reducing the power consumption of LTE networks by implementing different types of HetNets. Although the researchers tried to reduce the power consumption while trying to maintain a certain level of QoS, there is still more room for improvement and further power reduction while simultaneously increasing the provided data rate.

\subsection{Summary}

The following summarizes the previous works mentioned in this chapter. The studies all focused on reducing energy consumption while not concentrating sufficiently on the importance of increasing the data rate. Some suggested using sleep modes or switching off all or part of an eNB to reduce energy consumption. The downside of using sleep modes is that part of the eNB remains running. Figure 2.5 shows that a Pico-eNB consumes almost $50 \%$ of its power in sleep mode, which comes from the power amplifier, main supply and power supply. Consequently, the remaining power consumption of inactive eNBs can add up in eNB dense network. Table 2.3 shows the power consumption of different types of eNBs. Some of the HetNet switch on/off algorithms suggested switching off either the Macro-eNBs while keeping the small eNBs (Micro, Pico, or Femto-eNB) on, or the opposite. Building upon that, by switching off both Macro-eNBs and small eNBs, further energy reduction can be achieved 
with these algorithms. Furthermore, the types of networks employed in these papers were either Macro-eNB HoNets or Macro-Pico-eNB HetNets. Because some of the previously discussed papers fall under multiple of the above categories, we compiled Tables 3.1 and 3.2 , that show each paper with the category that it falls under. Table 3.1 shows the categorization of energy saving papers for HoNets, and Table 3.2 categorization of energy saving papers for HetNets. For example, paper [66] implemented static sleep mode algorithm for Macro-Micro-eNB HetNets, while paper 34 implemented dynamic switch on/off algorithm for Macro-eNB HoNets.

In this thesis, we proposed a new approach enabling LTE networks to deliver the promised high data rate of 1 Gbps. The approach proposed the replacement of all Macro-eNBs with a large number of Pico-eNBs covering the same area as the MacroeNBs. These Pico-eNBs are then managed by an adaptive network radio coverage that matches the UE distribution and demand by switching on/off Pico-eNBs. Usually, the objective of a switch on/off algorithm is to achieve the best possible energy conservation when switching off an eNB while maintaining full coverage. This goal can be achieved by minimizing the energy consumption while maintaining the data rate performance of the system. Altogether, switching on/off eNBs is an effective technique which can provide significant energy savings without compromising coverage while maintaining reasonable data rate as long as the switch on/off algorithm is designed properly.

In the current research, the new approach requirements and implementation within the SON framework are included. Also, a detailed simulation methodology that aims at providing higher data rate while reducing the power consumption of the network is provided. The methodology is applied to Pico-eNB HoNets. The results of the application of the new methodology have demonstrated its effectiveness and good correlation with all on Pico-eNB and Macro-eNB HoNets. It has been shown that there is a complex connection between coverage, data rate, power management, and 
interference. This connection has to be taken into account carefully to enable the switch on/off algorithm to save energy. To the best of our knowledge, the current research is the first of its kind to propose replacing Macro-eNBs entirely with a large number of Pico-eNBs capable of being switched on/off based on UE distribution with the objective of increasing the data rate and reducing the energy consumption while maintaining a certain acceptable outage. In Section 5.3.5, we executed a detailed simulation that compares our proposed switch on/off algorithm with two algorithms from the literature [26] and [44]. 
Table 3.1: Summary of related work for HoNets

\begin{tabular}{|c|c|c|c|c|c|c|}
\hline${ }_{\text {Category }}^{\mathrm{P}}$ & Dynamic & Static & Hybrid & $\mathrm{ON} / \mathrm{OFF}$ & Sleep mode & HoNet \\
\hline 26 & & $\checkmark$ & & $\checkmark$ & & $\checkmark$ \\
\hline 24 & $\checkmark$ & & & & $\checkmark$ & $\checkmark$ \\
\hline 27 & $\checkmark$ & & & & $\checkmark$ & $\checkmark$ \\
\hline 28 & $\checkmark$ & & & $\checkmark$ & & $\checkmark$ \\
\hline 29 & $\checkmark$ & & & $\checkmark$ & & $\checkmark$ \\
\hline 30 & $\checkmark$ & & & $\checkmark$ & & $\checkmark$ \\
\hline 31 & $\checkmark$ & & & & $\checkmark$ & $\checkmark$ \\
\hline 32 & $\checkmark$ & & & & $\checkmark$ & $\checkmark$ \\
\hline 33 & $\checkmark$ & & & & $\checkmark$ & $\checkmark$ \\
\hline 34 & $\checkmark$ & & & $\checkmark$ & & $\checkmark$ \\
\hline 35 & $\checkmark$ & & & & $\checkmark$ & $\checkmark$ \\
\hline 38 & & $\checkmark$ & & $\checkmark$ & & $\checkmark$ \\
\hline 39 & & $\checkmark$ & & $\checkmark$ & & $\checkmark$ \\
\hline 8 & & $\checkmark$ & & $\checkmark$ & & $\checkmark$ \\
\hline 40 & & $\checkmark$ & & $\checkmark$ & & $\checkmark$ \\
\hline 41 & & $\checkmark$ & & $\checkmark$ & & $\checkmark$ \\
\hline 42 & & $\checkmark$ & & $\checkmark$ & & $\checkmark$ \\
\hline 43 & & $\checkmark$ & & & $\checkmark$ & $\checkmark$ \\
\hline 44 & & $\checkmark$ & & $\checkmark$ & & $\checkmark$ \\
\hline 45 & & $\checkmark$ & & $\checkmark$ & & $\checkmark$ \\
\hline 46 & & $\checkmark$ & & $\checkmark$ & & $\checkmark$ \\
\hline 47 & & $\checkmark$ & & $\checkmark$ & & $\checkmark$ \\
\hline
\end{tabular}


Table 3.2: Summary of related work for HetNets

\begin{tabular}{|c|c|c|c|c|c|c|c|c|c|}
\hline Category & Dynamic & Static & Hybrid & $\begin{array}{l}\text { ON } \\
\text { OFF }\end{array}$ & $\begin{array}{l}\text { Sleep } \\
\text { mode }\end{array}$ & HoNet & $\begin{array}{l}\text { Macro } \\
\text { Pico } \\
\text { HetNet }\end{array}$ & $\begin{array}{l}\text { Macro } \\
\text { Micro } \\
\text { HetNet }\end{array}$ & $\begin{array}{l}\text { Macro } \\
\text { Femto } \\
\text { HetNet }\end{array}$ \\
\hline $37 \mid$ & & & $\checkmark$ & & $\checkmark$ & & & $\checkmark$ & \\
\hline 48 & & & & & & & $\checkmark$ & & \\
\hline 49 & $\checkmark$ & $\checkmark$ & & & $\checkmark$ & & $\checkmark$ & $\checkmark$ & \\
\hline 50 & & & & & & & $\checkmark$ & & \\
\hline 51 & & & & & & & $\checkmark$ & & \\
\hline 52 & & & & & & & $\checkmark$ & & \\
\hline 53 & & & & & & & $\checkmark$ & & \\
\hline 54 & $\checkmark$ & & & & $\checkmark$ & & $\checkmark$ & & \\
\hline$|55|$ & $\checkmark$ & & & & $\checkmark$ & & $\checkmark$ & & \\
\hline 56 & $\checkmark$ & & & & $\checkmark$ & & $\checkmark$ & & \\
\hline 57 & & $\checkmark$ & & $\checkmark$ & $\checkmark$ & & $\checkmark$ & & \\
\hline 58 & $\checkmark$ & & & & $\checkmark$ & $\checkmark$ & $\checkmark$ & & \\
\hline 59 & & $\checkmark$ & & $\checkmark$ & & & $\checkmark$ & & \\
\hline 60 & $\checkmark$ & & & $\checkmark$ & & & $\checkmark$ & & \\
\hline 61 & $\checkmark$ & & & & $\checkmark$ & & $\checkmark$ & & \\
\hline 62 & & & & & & & & $\checkmark$ & \\
\hline 63 & & & & & & & & $\checkmark$ & \\
\hline 64 & & & & & & & & $\checkmark$ & \\
\hline 65 & & & & & & & & $\checkmark$ & \\
\hline 66 & & $\checkmark$ & & & $\checkmark$ & & & $\checkmark$ & \\
\hline 67 & & & & & & & $\checkmark$ & $\checkmark$ & $\checkmark$ \\
\hline 68 & & & & & & & & & $\checkmark$ \\
\hline$|69|$ & $\checkmark$ & & & & $\checkmark$ & & & & $\checkmark$ \\
\hline 70 & & & & & & & & & $\checkmark$ \\
\hline 71 & & & & & & & & & $\checkmark$ \\
\hline 72 & & $\checkmark$ & & & $\checkmark$ & & & & $\checkmark$ \\
\hline 73 & & & & $\checkmark$ & $\checkmark$ & & & & $\checkmark$ \\
\hline
\end{tabular}




\section{Chapter 4}

\section{Proposed Vision and Algorithms}

In this chapter, we first introduce the proposed vision of replacing Macro-eNBs with a large number of Pico-eNbs. We formulate an optimization problem with an objective function of minimizing the overall consumed power while supporting user demand with acceptable quality of service. Then, we discuss a way of implementing the optimization problem by designing two switch on/off algorithms. Also, we go through the network design approach which contains information about propagation models, the network model and the LTE frame structure. Furthermore, we define in detail two switch on/off algorithms that were implemented in this thesis. Finally, we mention the different UE distribution and mobility models that are used to generate the simulations in the next chapter.

\subsection{Overview of Proposed Vision}

LTE networks have to adapt to the daily or weekly change of the demographics of UE. This requirement cannot be fulfilled by the traditional large coverage Macro-eNBs. Switching off a Macro-eNB affects a large area and may introduce an outage in that

area. As shown in Figure 2.3 (see Section 2.3), HetNets were introduced by 3GPP. These types of eNBs are mixed in an operating network. Using small eNBs like Micro, 
Pico and Femto can enhance coverage and capacity. It is foreseen that there will be a large number of these small eNBs in operation, each of them covering an area that is significantly smaller than that of a Macro-eNB. Each of the small eNBs corresponds to a number of objects with attributes and measurements to manage. At the same time, each Micro/Pico-eNB is a node by itself and the requirements to manage them are similar to Macro-eNBs. UE will connect to the Macro-eNB if it is not able to find a small eNB to connect to it.

Every Pico-eNB connects with the core through their S1 interface. Therefore, implementing small eNBs raises new challenges for mobile operators backhaul planning. Even though fixed line backhaul solutions provide optimal capacity, operators are generally limited by the lack of fiber availability and the need to deploy small eNBs in areas with limited wireline access. To backhaul those eNBs, wireless solutions can be used in such cases. Small eNBs' backhaul traffic is generally lighter than that of Macro-eNBs, but traffic levels are expected to increase. The backhaul connection may quickly become the bottleneck due to the concentration of users close to the site.

Nokia networks conducted experiments to demonstrate the deployment of LTE small cell clusters under a Macro-eNB. These experiments showed that capacity and end-user experience could be improved, given that each small cell has a proper SON algorithm to adapt itself to its surroundings. The small cells should have a degree of Radio Frequency (RF) isolation relative to the Macro-eNB network and should not be placed in locations where the UE to be served by Pico-eNBs will be within Line-of-Sight (LOS) of the Macro-eNB. Buildings and the use of directional antennas can often provide isolation between the Macro-eNB and small cell networks. Radio link failures and "ping pong" handover effects can occur in the cell areas where the downlink signals from the Macro-eNB and the small cells are at the same level as the $\mathrm{UE}$ [74.

The main objective of the current research is to enable LTE networks to provide 
users with very high data rates while simultaneously trying to reduce power consumption. The approach is based on replacing Macro-eNBs with a large number of Pico-eNBs which is expected to eliminate the bottleneck effect of connecting PicoeNBs to a Macro-eNB. Another advantage of the approach of using only Pico-eNBs is the elimination of the high power consumption of Macro-eNBs [75]. The current Macro-eNBs can be used for control signals and relay network information to the core. Also, Macro-eNBs can be used when a UE cannot find a Pico-eNB to connect to, for voice communications, and to connect high mobility UE. The latter is outside the focus of this thesis, as this thesis is on replacing Macro-eNBs with large number of Pico-eNBs. Also, as mentioned in Section 3.3, several other researchers have worked on energy saving in Macro-Pico-eNB HetNets. Furthermore, the control link impact on the system power consumption is negligible compared to the data link.

Implementing Pico-eNBs allows network operators to switch on/off eNBs based on traffic estimation and statistics. Migration of UE from one location to another during the day or from day to day will trigger switch on/off algorithms that will shift resources according to UE distribution. Figure 4.1 schematically presents the proposed approach and shows that it is possible to have some coverage holes in the network; a proper network planning thus needs to be done to keep these coverage holes under a certain acceptable value.

\subsubsection{Proposed Metric}

With the increase in the number of eNBs in the network, network operators can observe a network area with eNBs that share the same bandwidth over a long period of time and measure the amount of power used to deliver a certain number of bits to UE. Therefore, just observing the data rate or power consumption alone does not provide a clear picture of the performance of the network.

The total energy per bit (TEPB) measurement unit $\left(\frac{\text { Joules }}{b i t}\right)$ is proposed to compare 


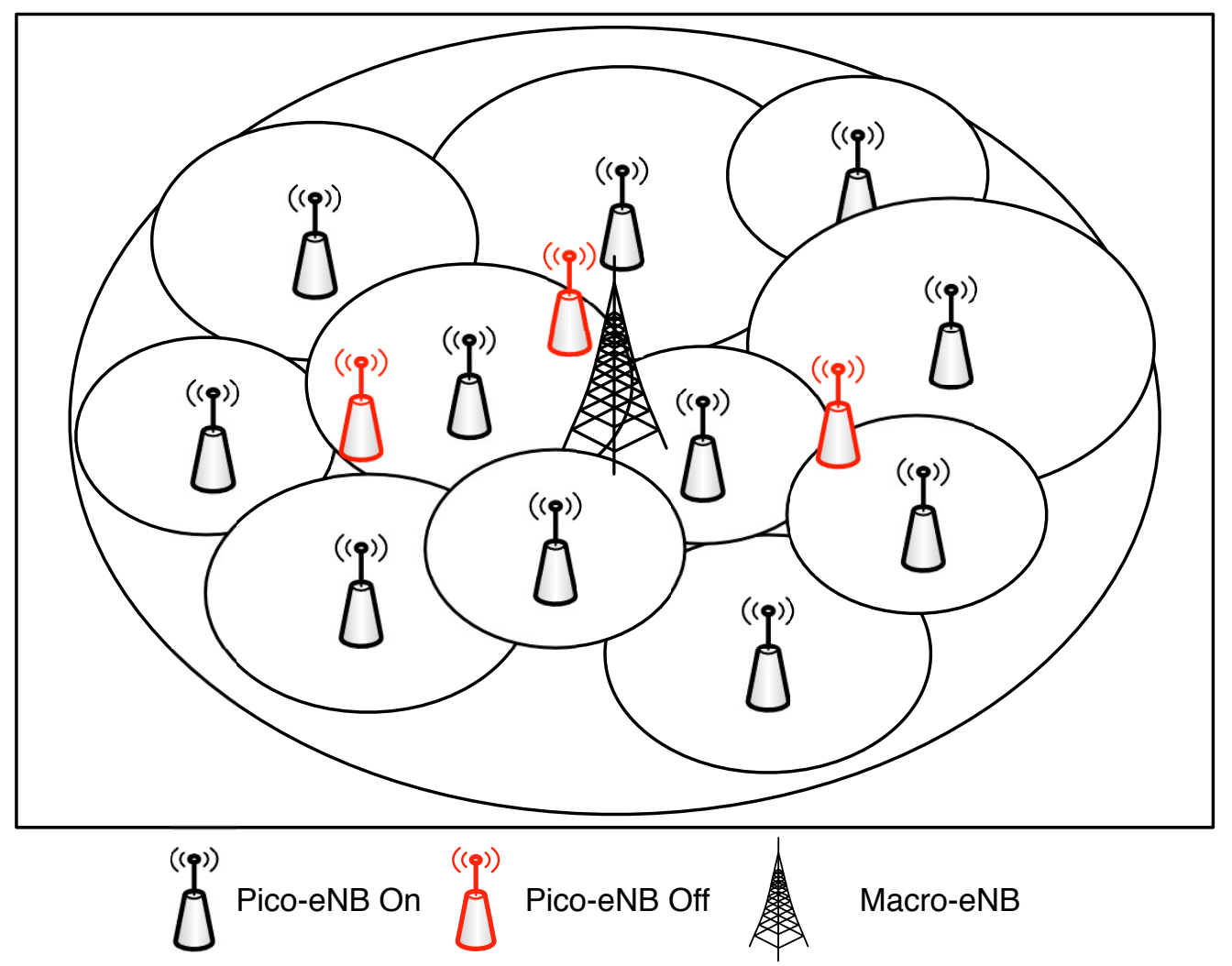

Figure 4.1: Proposed vision: LTE network with only Pico-eNBs that can be switched on/off

the performance of different energy saving strategies fairly, which is calculated as shown in Equation 4.1). Consider a given geographical area $(A)$. The area is served by a number of Pico-eNBs $(M)$, and all Pico-eNBs share the same bandwidth $(B W)$. We observe the system over time $(T)$, which represents the traffic fluctuations within $A$. When comparing two energy saving protocols, we fix $A, B W$ and $T$, then we calculate the total number of bits received correctly by all Pico-eNBs during T. TEPB is the ratio between the total power consumption by all Pico-eNBs and the number of bits received. The total consumed power includes the transmitted power, processing 
power and power used for house keeping functions such as air conditioning.

$$
T E P B=\frac{P}{\eta \cdot A \cdot T \cdot B W}
$$

Where:

- $P$ is the total power consumption by the network in Watts.

- $A$ is the network area in $\mathrm{m}^{2}$.

- $T$ is the time interval in sec.

- $B W$ is the network bandwidth in $H z$.

- $\eta$ is the network average spectral efficiency in $\left(b p s / H z / m^{2}\right)$ which is the amount of information that can be transmitted over a given bandwidth and can be calculated by using Shannons capacity $\eta=\log _{2}(1+S I N R)$.

In the reminder of this section, the change in network TEPB for different PicoeNB densities $\left(\lambda_{b}\right)$ and a fixed UE density $\left(\lambda_{u}\right)$ was investigated. This is of practical relevance, because it corresponds to testing to what extent network operators can deploy large number of Pico-eNBs. Three different network regimes were considered in terms of $\lambda_{b}$, which are:

Low Pico-eNB density: $\left(\lambda_{b} \ll \lambda_{u}\right.$, so the probability that an eNB is active is close to $\left.1\left(P_{a} \approx 1\right)\right)$

In the case of lower $\lambda_{b}$, almost all Pico-eNBs are active $\left(P_{a}>0.99\right.$ when eNBUE density ratio $(\rho \leq 0.1))$. Pico-eNBs are limited by the number of UE that they can serve at a certain time, which will be discussed in more details in Section 4.3.7. That can lead to over loading the network, increasing the delay and reducing the network throughput. On the other hand, the network power 
consumption in this case is lower. The lower throughput leads to an increase in TEPB as $T E P B \propto \frac{1}{\text { Throughput }}$.

\section{High Pico-eNB density: $\left(\lambda_{b} \gg \lambda_{u}\right)$}

In the case of higher $\lambda_{b}$, all UE are being served and $P_{a} \approx \frac{1}{\rho}$. By increasing the Pico-eNB density, the network throughput can increase as the distance between the UE and Pico-eNB is smaller. Another reason for the increase in the network throughput is that the number of UE served by each Pico-eNB is lower, which means less delay and lower number of dropped UE.

At the same time, when increasing the number of Pico-eNBs in the network, the power consumption increases. The increase in the number of Pico-eNBs increases the network throughput but at the same time, the $P_{a}$ decreases, which leads to underutilized Pico-eNBs. The increase in power consumption in relation to the increase in network throughput leads to an increase in TEPB.

\section{Medium Pico-eNB density: $\left(\lambda_{b} \sim \lambda_{u}\right)$}

In this last case, the Pico-eNB density and the UE density are proportional, which is a more practical regime for Pico-eNB networks [76]. For this case, the TEPB measurement plays a big factor in the decision of when to add more Pico-eNBs or remove some.

A simulation was constructed to test to what extent the network can be split into smaller cells while maintaining an acceptable network TEPB. A network area of $4 \mathrm{~km}^{2}$ was studied in all simulations and the same simulations were repeated for 250 , 500, 1000, 1500 and 2000 UE uniformly distributed in the network. All simulations start with one large cell at the centre of the network and the TEPB of the network was calculated to achieve a reference point. Then, each cell was split into smaller cells which are arranged in a square lattice. The number of eNBs used in each simulation 
was changed with the following values: $\{1,4,16,36,64,100,144,196,256,324,400\}$. In order to maintain the Inter Cell Interference (ICI) while allowing the new eNBs to cover the same area, the transmission power of all eNBs was reduced accordingly.

For example, when 1 Pico-eNB was at the centre of the network, the Pico-eNB's transmit power was set to $36 \mathrm{dBm}$, while when replacing that Pico-eNB with 64 Pico-eNBs, the new transmit power was changed to $18 \mathrm{dBm}$. These values were chosen in order to have a close to $0 \%$ outage, which means almost all UE in the whole area under study can connect to a Pico-eNB. In all simulations, each Pico-eNB is considered to consume 35 Watts.

The simulation was repeated 10 times for every number of UE to produce Figures. 4.2 and 4.3 . Figure 4.2 shows a detailed example of the effect of cell splitting on the throughput and the power consumption of the network for 500 UE uniformly distributed in the network. The network throughput increases with the increase in the number of eNBs as the distance between the UE and the serving eNB is decreasing. The increase in the throughput is also due to the reduction of the number of UE served per eNB. On the other hand, the power consumption of the network increases linearly with the increase in the number of eNBs in the network.

Figure 4.3 presents the effect of cell splitting on TEPB for different numbers of UE uniformly distributed in the network. These results show an improvement in the TEPB of the network due to splitting large cells into smaller cells. The best network TEPB for 250 UE can be achieved when splitting the network into 36 small cells, whereas for the rest it was 64 small cells, at which point the TEPB starts to increase. The increase in TEPB is due to the fact that the increase in the network throughput is not high enough to accommodate the increase in power consumption. The latter is more evident in the case of $250 \mathrm{UE}$, specially when the number of eNB in the network is higher than the number of UE.

Figure 4.4 shows the Channel Quality Indicator (CQI) maps for two network 


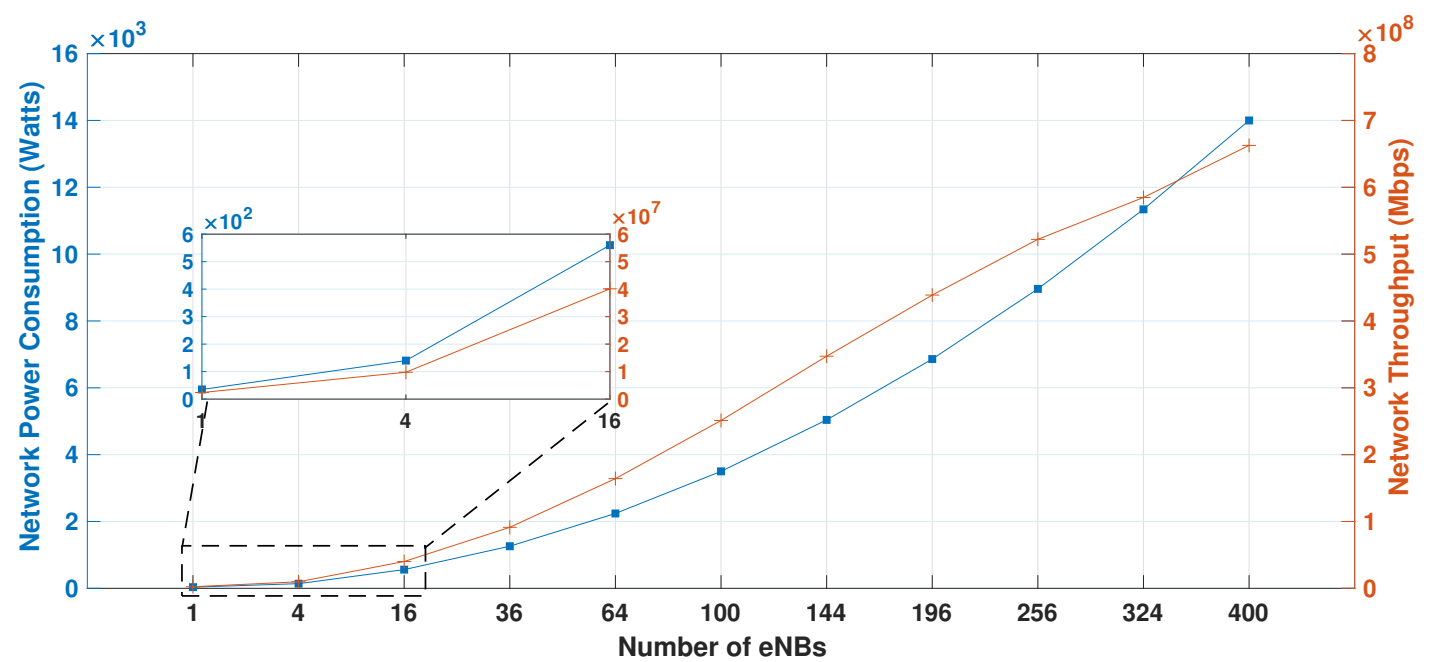

Figure 4.2: Network power consumption vs network throughput for $500 \mathrm{UE}$



Figure 4.3: Effect of cell splitting on TEPB

setups with 1 eNB at the centre (Figure 4.4a) and the network with the large cell replaced with 64 eNBs (Figure 4.4b). Also, Figure 4.4b shows an increase in the lower CQI values (between 0 and 5) compared to Figure 4.4a. That change is due to the increase in ICI caused by higher $\lambda_{b}$ values and also due to the reduction of the transmit power of all Pico-eNBs. The CQI values range between 0 and 15, and an outage occurs if a $\mathrm{UE}$ cannot receive at least $C Q I=1$. More details about CQI 


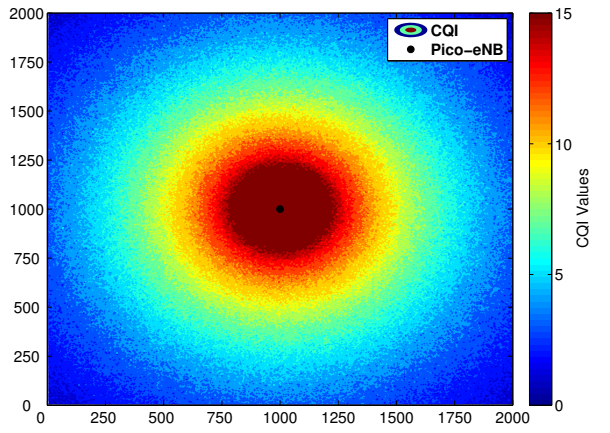

(a) 1 eNB

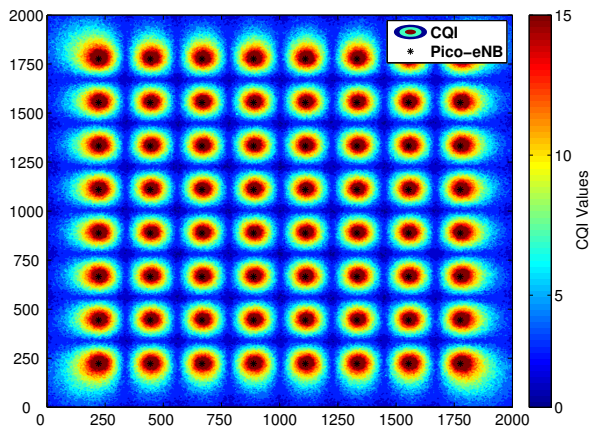

(b) $64 \mathrm{eNBs}$

Figure 4.4: Network setup with 1 and 64 eNBs, also showing the range of each eNB calculation will follow in Section 4.3.3.

By splitting larger cells into smaller cells for the same number of users, both network throughput and power consumption increase at which point the amount of increase in throughput is not enough to cancel the effect of the increase in power consumption. Therefore, it can be concluded from this simulation that in order to achieve the best network performance (for the given network setup) in terms of TEPB, network providers cannot keep splitting cells indefinitely. The optimal number of Pico-eNBs that can be supported by a network needs to take into account both factors (throughput and power consumption) to minimize the TEPB.

Based on the above results, we can derive general guidelines for optimal cell splitting by specifying the factors that need to be taken into account when splitting cells:

- Specify the type of eNBs that are used, to find the power consumption and maximum number of UE that can connect to that type of eNB.

- Find the maximum number of UE that are available in the area under study and where they are concentrated. This can be used to decide where to increase the number of eNBs in the network.

- Find the UE load in the area of interest. This can be done by collecting UE 
information over a period of time.

- When splitting large cells into smaller cells the, ICI increases. Therefore, the transmit power of the eNBs needs to be reduced in order to minimize the ICI.

\subsubsection{Implementation of the Proposed Vision}

The evolution of current LTE networks into the proposed vision that is expected to happen in the not-so-distant future is discussed in this section. In the early stages of implementation, current LTE networks will continue as they currently do, while Pico-eNBs are deployed as needed to accommodate the UE requirements. The current Macro-eNB under whose coverage the new Pico-eNBs were added will be used for control signals and when UE cannot find a Pico-eNB to connect to, as discussed in Section 4.1

The new added Pico-eNBs can connect to the core through fiber optics, if available, or by relaying information through neighboring Pico-eNBs or the current Macro-eNB under whose coverage they lie. Eventually, the network will have a large number of Pico-eNBs that cover every part of the network and the need for the Macro-eNB will be minimal. With the increase in the number of Pico-eNBs in the network, the need for energy reduction increases, at which point the role of the switch on/off algorithm becomes more significant. Some areas of the network (such as rural areas) may have a naturally occurring small number of UE. In these areas, the current Macro-eNB setup could be more than sufficient to provide UE with the required QoS.

\subsubsection{Backhaul and Handover Challenges}

With the introduction of a large number of Pico-eNBs, the network capacity will increase but on the other hand, backhauling traffic from Pico-eNBs to the core will 




Figure 4.5: LTE system model with various possible types of Pico-eNB backhauling

be a major issue. A more flexible and scalable backhaul network architecture is required. For example, the deployment of Pico-eNBs requires access to infrastructure with wired network backhauling. Because of that, network operators need to plan the backhaul of the network carefully to guarantee the most cost effective and best performance network setup. A network can include a mixture of wireless and wired backhaul technologies. Some Pico-eNBs may connect to the core network with dedicated interfaces, while other Pico-eNBs may use wireless connections to connect to the core network. In other cases, some Pico-eNBs with lower load can be used to relay data to the core as an alternative interface. Figure 4.5, shows the system model with the various possible types of Pico-eNBs backhauling.

There are many wireless backhauling technologies that can be used, such as millimeter wave and microwave radios but they both require line of sight. In the case of Non Line of Sight (NLoS), RF-based backhaul can be used to carry traffic, which can be done on the licensed or unlicensed bands. The unlicensed band is less expensive 
but it is more crowded while the licensed band is more efficient and secure [77].

Another important factor that needs to be considered is handovers, which is essential to provide a seamless uniform service for UE when moving between cells. Handovers are effective for traffic load balancing, that can be achieved by shifting UE at the border of overlapping cells from more congested to less congested cells. That said, this comes at the expense of system overhead, which is likely to be significant when replacing Macro-eNBs with large number of Pico-eNBs. Pico-eNBs are mainly used for relatively low mobility UE using data intensive applications. A high mobility UE would probably be connected through a Macro-eNB to reduce overhead caused by handovers between Pico-eNBs. Furthermore, the probability of handover failure increases the probability of user outage [78].

\subsection{Optimization Problem Formulation}

In order to reduce the energy consumption of the network while maintaining the desired high data rate, a switch on/off algorithm is needed. The goal of the switch on/off algorithm is to minimize the TEPB so that the network can adapt to the fluctuation in traffic demand throughout the day. As a first step, we formulated this as an optimization problem. The switch on/off algorithm optimization problem can be formulated as a binary integer linear programming (BILP) problem as shown in Equations 4.2 to 4.5.

$$
\operatorname{minimize} \frac{\sum_{i=1}^{|I|} P_{i} x_{i}}{\sum_{j=1}^{|J|} R_{j}},
$$

subject to: $\quad$ Outage probabilty $\leq Z$,

$$
N_{i} \cdot x_{i}<\text { number of } R B_{i} \quad \forall i \in I
$$




$$
x_{i} \in\{0,1\}
$$

Where:

- $I$ is the set of eNB that are installed in the network.

- $J$ is the set of UE in the network.

- $P_{i}$ represents the power consumption (in Watts) for eNB $i \in I$.

- $R_{j}$ represents the number of successfully transmitted bits in downlink direction to UE $j \in J$.

- $x_{i}$ is a binary variable representing the status (i.e. on or off) of a Pico-eNB. Its value is 0 when Pico-eNB $i \in I$ is off, otherwise 1 .

- $N_{i}$ represents the maximum number of UE that can be served by Pico-eNB $i \in I$.

- $Z$ represents the percentage of network outage. The value of $Z$ depends on the location of eNBs in the network that are on and off, which relies on $x_{i}$.

The value of $P_{i}$ can be chosen based on the type of eNB used. As discussed in Section 2.4, if an eNB is running (whether it has an active UE or not) it will be consuming most of its power. Therefore, for simplicity and without loss of generality, the power consumption per Pico-eNB is set to a constant value (35 Watts).

In LTE specifications, UE cannot be served if the received SINR is not large enough to support QPSK modulation. This is the network outage criterion. In Equation (4.2), $Z$ represents the fraction of locations within the area under consideration where LTE UE cannot be served. The value of $Z$ is affected by the propagation characteristics (including random fluctuations), and it also depends on the interference details and the locations of eNBs. When switching eNBs on/off, the distribution of eNBs in 
the network changes which in return affects the value of $Z$. Therefore, $Z$ is affected mainly by which eNB is switched on/off (i.e. $x_{i}$ ). Consequently, with a larger number of eNBs in the network, the value of $Z$ becomes very difficult to compute analytically as will be shown in Section 4.3.5. Going back to the low Pico-eNB density network regime discussed in Section 4.1.1, some UE might fall in a low SINR location. If a location in the network cannot be served with at least a $C Q I=1$, an outage occurs.

In general, integer programming problems are non-deterministic polynomial-time hard (NP-hard) [79]. In spite of that, the problem can be solved efficiently for a relatively small number of eNBs. With a larger number of eNBs, however, the complexity of the problem increases since the number of possible eNB combinations increases exponentially $\left(N_{c o m b}=2^{N}-1\right)$. To solve this issue, two approximate switch on/off algorithms are proposed in this thesis. Section 4.3 .5 discusses in more details the complexity of formulating the system as an optimization problem.

\subsection{Design Approach}

In this section, the network setup is discussed, including the replacement of MacroeNBs with several Pico-eNBs to service the same coverage area. The Element Management System/Network Management System (EMS/NMS) divides the system into groups of $N$ Pico-eNBs, which are chosen to create a cluster of neighboring PicoeNBs. Any Pico-eNB distribution can be used in the network as the algorithm can be applied to any network design. Each Pico-eNB is connected to the core through the S1 interface. The EMS/NMS collects all Pico-eNBs' information from the MME and saves them in a database to be used later by the SON functions. 


\subsubsection{LTE Propagation Path-Loss Models}

Path-loss propagation model is an important component in the analysis and design of the link budget of a wireless communication system. Path-loss depends on several factors, such as free-space loss, terrain contours, environment, the distance between the transmitter and the receiver, in addition to the height and location of antennas [80]. The path-loss $(P L)$ is the attenuation (in $d B$ ) between the transmitted $\left(P_{t x}\right)$ and received $\left(P_{r x}\right)$ power, including the effect of antenna gains $\left(G_{\text {antenna }}\right)$ as shown in Equation (4.6). Equation 4.7 defines the power received by a UE as the difference between the transmitted power and the environment path-loss [81]. The following sections explain the Macro and Pico-eNBs' propagation models that are used in this research.

$$
\begin{gathered}
P L=10 \log _{10}\left(\frac{P_{t x} G_{e N B} G_{U E}}{P_{r x}}\right) \\
P_{r x}(d)=P_{t x}+G_{e N B}+G_{U E}-P L(d)
\end{gathered}
$$

Where:

- $P_{r x}$ is the received power from the serving eNB.

- $P_{t x}$ is the transmitted power from the serving eNB.

- $G_{e N B}$ denotes the eNB antenna gain in $d B i$.

- $G_{U E}$ denotes the UE antenna gain in $d B i$.

- $P L(d)$ is the path-loss between the transmitter (eNB) and receiver (UE) separated by distance $\mathrm{d}$ in $\mathrm{km}$.

\subsubsection{Macro-eNB Propagation Model}

The Macro-eNB propagation model (Equation (4.9) that is used in this research is applicable to scenarios in urban and suburban areas where the buildings have a nearly 
uniform height [1].

$$
P L=40\left(1-4 \times 10^{-3} \cdot h\right) \log 10(d)-18 \log 10(h)+21 \log 10(f)+80 d B
$$

Where:

- $h$ is eNB antenna height in $\mathrm{m}$, measured from above the rooftop level.

- $d$ is eNB-UE separation in $\mathrm{km}$.

- $f$ is the carrier frequency in $\mathrm{MHz}$.

In [1], it was assumed that the Macro-eNB antenna is positioned at $15 \mathrm{~m}$ above the rooftop, and a carrier frequency of $2 \mathrm{GHz}$ is used, the Macro-eNB path-loss $\left(P L_{\text {Macro }}\right)$ (in $d B$ ) can be expressed as shown in Equation 4.9):

$$
P L_{\text {Macro }}(d)=128.1+37.6 \log 10(d)+F_{s}
$$

Where, $F_{s}$ is the log normal shadow fading with standard deviation $10 \mathrm{~dB}$. The Macro-eNB propagation model from Equation (4.9) gives the worst case propagation scenario and is valid for Non Line of Sight (NLoS) cases only. This model is not very accurate for short distances as it is designed for distances ranging from a few hundred meters to a few kilometers.

\subsubsection{Pico-eNB Propagation Model}

As shown in Equation (4.10), the Pico-eNB propagation model $P L_{\text {Pico }}$ is using a carrier frequency of $2 \mathrm{GHz}$ and is expressed in $d B$.

$$
P L_{\text {Pico }}(d)=140.7+36.7 \log 10(d)+F_{s}
$$




\subsubsection{SINR Calculation}

In information theory and telecommunication engineering, the SINR is a quantity used to estimate the theoretical rate of information transfer in wireless communication systems. The SINR is defined as the power of the signal of interest divided by the sum of the interference power, and the power of background noise, as shown in Equation (4.11). If the noise power is zero, then the SINR reduces to the Signal-toInterference Ratio (SIR). On the other hand, if the interference is zero, the SINR is reduced to the Signal-to-Noise Ratio (SNR), which can be used to develop mathematical models of wireless networks such as cellular networks [81. Equation 4.11was used to calculate the SINR, which takes into account the received interfering signal from all surrounding eNBs in the network. Therefore, all eNBs are considered to use the same bandwidth $(20 \mathrm{MHz})$.

$$
\operatorname{SINR}\left(d_{j}\right)=\frac{P_{r x}\left(d_{j}\right)}{\sum_{i \neq j} P_{r x}\left(d_{i}\right)+T_{0}} \quad(\forall i \in I)
$$

Where:

- $P_{r x}$ is the received power from the desired eNB.

- $j$ is the index of the serving eNB

- I set of eNB that are installed in the network.

- $T_{0}$ is the thermal noise.

By applying Equation (4.7) in Equation 4.11, we can achieve the SINR at distance $d$ as shown in Equation 4.12.

$$
\operatorname{SINR}\left(d_{j}\right)=\frac{P_{t x_{j}} G_{e N B_{j}} G_{U E_{j}} P L\left(d_{j}\right)^{-1}}{\sum_{i \neq j}\left(P_{t x_{i}} G_{e N B_{i}} G_{U E_{i}} P L\left(d_{i}\right)^{-1}\right)+T_{0}}
$$


Table 4.1: Lookup table for mapping SINR estimate to CQI index 3]

\begin{tabular}{lccc}
\hline CQI index & MCS & Code Rate & SINR estimate $(d B)$ \\
\hline 1 & QPSK & 0.101449 & -6.7 \\
2 & QPSK & 0.101449 & -4.7 \\
3 & QPSK & 0.162319 & -2.3 \\
4 & QPSK & 0.318841 & 0.2 \\
5 & QPSK & 0.442210 & 2.4 \\
6 & QPSK & 0.568116 & 4.3 \\
7 & 16QAM & 0.365217 & 5.9 \\
8 & 16QAM & 0.469565 & 8.1 \\
9 & 16QAM & 0.563768 & 10.3 \\
10 & 64QAM & 0.484058 & 11.7 \\
11 & 64QAM & 0.600000 & 14.1 \\
12 & 64QAM & 0.692754 & 16.3 \\
13 & 64 QAM & 0.760870 & 18.7 \\
14 & 64QAM & 0.888406 & 21.0 \\
15 & 64QAM & 0.888406 & 22.7 \\
\hline
\end{tabular}

From Equation 4.10), we get $P L_{P i c o}=10^{(140.7 / 10)} d^{3.67} f$, where we can assume $c=10^{(140.7 / 10)}$ and $\alpha=3.67$ which is the path-loss exponent. We can simplify Equation (4.10) into $P L_{\text {Pico }}(d)=c d^{\alpha} f$. Substituting $P L\left(d_{j}\right)$ and $P L\left(d_{i}\right)$ with $P L_{\text {Pico }}$ in Equation 4.12 we get Equation (4.13), which will be used later in calculating the probability of outage.

$$
\operatorname{SINR}\left(d_{j}\right)=\frac{P_{t x_{j}} G_{e N B_{j}} G_{U E_{j}} c^{-1} d_{j}^{-\alpha} f_{j}^{-1}}{\sum_{i \neq j}\left(P_{t x_{i}} G_{e N B_{i}} G_{U E_{i}} c^{-1} d_{i}^{-\alpha} f_{i}^{-1}\right)+T_{0}}
$$




\subsubsection{CQI Calculation}

The UE measures the downlink reference signal and determines the CQI value. Each CQI value corresponds to the highest Modulation and Coding Scheme (MCS) that allows the UE to demodulate and decode the required Transport Block (TB) with maximum Block Error Rate (BLR) (10\%). These CQI values are sent to the serving eNB periodically by the UE on the uplink direction. The eNB selects a suitable MCS for the downlink transmission for that particular UE. Table 4.1 shows the CQI index with corresponding parameters [3]. For example, if a UE measures its SINR of1.2 $d B$, then the UE reports a CQI 4 to the serving eNB. If the channel quality is very low and a UE couldn't achieve $C Q I=1$, then the connection will be dropped and that will be considered as an outage. The CQI reporting frequency and accuracy depends on the algorithm used in the UE handset. A fast moving UE can report a certain CQI value to the serving eNB and by the time the eNB receives that value and processes it, the UE location might have changed which in return might change the CQI value. Therefore, a proper CQI reporting algorithm needs to be implemented, which is a field of research on its own.

\subsubsection{Network Model}

We consider a cellular network, as shown in Figure 2.1, where eNBs and UE are distributed in the network with densities $\lambda_{b}$ and $\lambda_{u}$ respectively. We also consider the downlink transmission and assume that each UE is served by the nearest eNB.

Due to the independent locations of eNBs and UE, there may be some eNBs that do not have any UE to serve. ENBs that have UE to serve are called active eNBs with a probability $P_{a}$ given by Equation 4.14 [82].

$$
P_{a}=1-\left(1+\frac{1}{\mu \rho}\right)^{-\mu}
$$


Where:

- $P_{a}$ is the probability that an eNB is active.

- $\mu$ is a constant related to the cell size distribution, where $\mu=3.5$ [83].

- $\rho$ is the eNB-UE density ratio $\rho=\frac{\lambda_{b}}{\lambda_{u}}$.

The probability that a randomly chosen eNB does not have any UE in its coverage area is $P_{0}=1-P_{a}$. On the other hand, the probability that a randomly chosen UE is successfully assigned a RB at a given time and is served by the nearest eNB is given by Equation 4.15).

$$
P_{s}=\rho P_{a}
$$

An active eNB may have one or more UE under its coverage, and the eNB will randomly choose one or a group of UE to serve in a radio frame. Assuming a fixed data rate, the network throughput is determined by the outage probability. An outage occurs if the received SINR falls below a certain threshold $\gamma$, which means that an eNB cannot service a $\mathrm{UE}$ with at least $C Q I=1$. The network outage probability is given by Equation (4.16) [84].

$$
P_{\text {out }}=\mathbb{P}(S I N R \leq \gamma)
$$

\subsubsection{Calculating Probability of Outage at Distance $d$}

Because all eNBs have identical $P_{t x}, G_{e N B}, G_{U E}$ and $C$, also by assuming that the thermal noise is negligible, then the SINR calculation in Equation 4.13) can be rewritten as $S I N R=1 / F$ where 85 :

$$
F=\frac{\sum_{i \neq j}\left(d_{i}^{-\eta} f_{i}^{-1}\right)}{d_{j}^{-\eta} f_{j}^{-1}}
$$


Because the numerator of $F$ is a sum of log-normally independent and identically distributed (iid) random variables, and the denominator is also a log-normally distributed random variable, $F$ can be approximated by a log-normal random variable. Using the Fenton-Wilkinson method [86], we can find the mean $\left(m_{f}\right)$ and standard deviation $\left(s_{f}\right)$ of $F$ for any UE at distance $d$ from the serving eNB which are different than the mean $\left(\mu_{i}\right)$ and standard deviation $\left(\sigma_{i}\right)$ of each random variables separately, as shown in Equations 4.18 to 4.22, where $a=\frac{\ln 10}{10}$ (Appendix A.

$$
\begin{gathered}
m_{f}=\frac{1}{a} \ln \left(y_{f}(d, \eta) H(d, \sigma)\right) \\
s_{f}=2\left(\sigma^{2}-\frac{1}{a^{2}} \ln (H(d, \sigma))\right)
\end{gathered}
$$

Where:

$$
\begin{gathered}
H(d, \sigma)=e^{a^{2} \sigma^{2} / 2}\left(G(d, \eta)\left(e^{a^{2} \sigma^{2}}-1\right)+1\right)^{\frac{1}{2}} \\
G(d, \eta)=\frac{\sum_{i} d_{i}^{-2 \eta}}{\left(\sum_{i} d_{i}^{-\eta}\right)^{2}} \\
y_{f}(d, \eta)=\frac{\sum_{i} d_{i}^{-\eta}}{d^{-\eta}}
\end{gathered}
$$

To find the outage probability of a UE at a distance $d$ from its serving eNB, we use the Q-function:

$$
\begin{gathered}
\mathbb{P}(S I N R<\gamma)=1-\mathbb{P}\left(\frac{1}{\gamma}>F\left(m_{f}, s_{f}\right)\right) \\
\mathbb{P}(S I N R<\gamma)=1-\mathbb{P}\left(10 \log 10\left(\frac{1}{\gamma}\right)>10 \log 10(F)\right) \\
\mathbb{P}(S I N R<\gamma)=Q\left[\frac{10 \log 10\left(\frac{1}{\gamma}\right)-m_{f}}{s_{f}}\right]
\end{gathered}
$$




\subsubsection{Complexity of the network model}

Because interference is the main factor in SINR calculation, as shown in Equation (4.13), in most wireless networks, it is important to distinguish the interference statistics. Network geometry and path-loss are the two main determinants of the interference. For certain types of eNB distributions, closed form results for interference and SINR are available, which can be used to determine the network performance, as the analysis above shows.

The exact characterization of the interference or SINR for general eNB distributions is a very challenging problem. Since the focus of this thesis is on the switching on/off algorithm to enhance the network performance, the network structures are chosen because they are easier to manage, not necessarily because they are the most realistic ones. Analytical methods are best used when applied to simple models, specially when the models used are more ideal cases. Two of the most common network structures used are hexagonal cells (triangular lattice) and Poisson Point Process (PPP) because of their simplicity and analytical tractability [87].

If we consider the initial network structure with one of these two structures, calculating the network interference distribution is easy, as it is the sum of the log-normal random variables, as shown in Equations (4.18) to (4.22). However, once the switch on/off algorithm starts switching on/off Pico-eNBs to accommodate the change in UE distribution and demand, the network structure falls somewhere between triangular lattice and PPP. Consequently, the numerator of $F$ from Equation (4.17) becomes the sum of non-iid log-normal random variables which is in return complex to formulate analytically, thus it needs to be simulated as explained in the next chapter. 


\subsubsection{Network Spectral Efficiency}

The network spectral efficiency is defined as the average number of successfully transmitted bps/Hz/unit - area, as given by Equation (4.26). Similarly, the average spectral efficiency per UE can be calculated by Equation (4.27) [88. These two spectral efficiency metrics are affected by the eNB-UE density in different ways, and subsequently, important design guidelines can be drawn.

$$
\begin{aligned}
& R_{a}=\lambda_{b} P_{a}\left(1-P_{\text {out }}\right) R_{0} \\
& R_{u}=\rho P_{a}\left(1-P_{\text {out }}\right) R_{0}
\end{aligned}
$$

Where:

- $R_{a}$ is the network area spectral efficiency in bps/Hz/unit - area.

- $\lambda_{b} P_{a}$ is the density of active eNBs.

- $P_{\text {out }}$ is the network outage probability.

- $R_{0}=\log _{2}(1+\gamma)$ which is the Shannons capacity limit.

- $R_{u}$ is the average spectral efficiency per UE in bps/Hz/unit - area.

\subsubsection{LTE Frame Structure}

The LTE frame structure is shown in Figure 4.6, the total frame duration is $10 \mathrm{~ms}$. A frame contains 10 subframes and each subframe is composed of 2 time slots $(0.5 \mathrm{~ms})$. Another important unit in the LTE frame structure is the resource block (RB), which is made up of one time slot, and each UE can be dynamically allocated a minimum of 2 RBs in a subframe. Table 4.2 shows how many RBs are available in each bandwidth. 


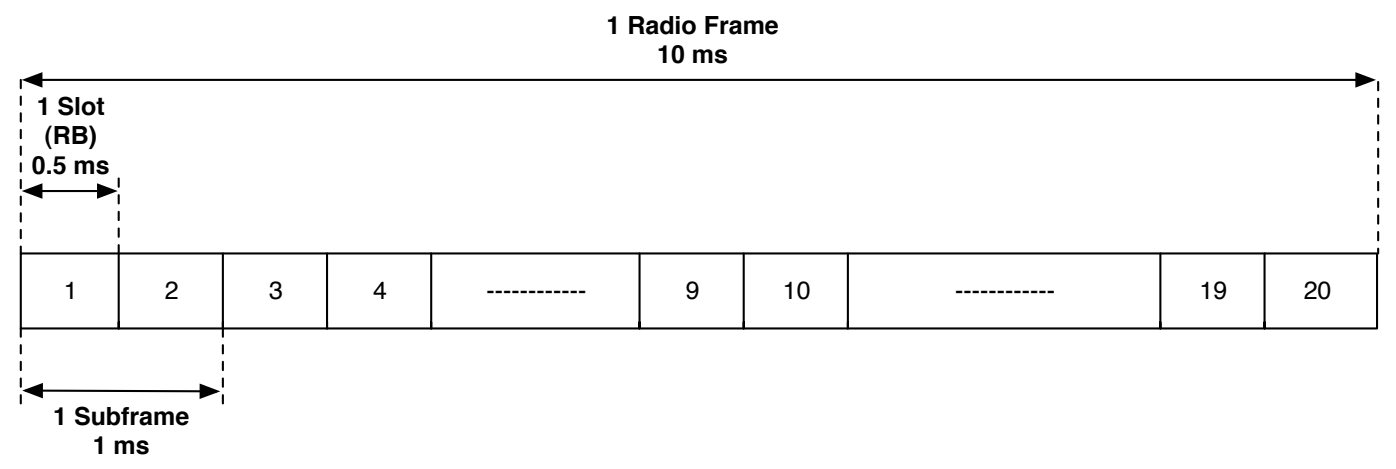

Figure 4.6: LTE frame structure

Table 4.2: Number of resource blocks for different bandwidths [3]

\begin{tabular}{lcccccc}
\hline Bandwidth (MHz) & $\mathbf{1 . 4}$ & $\mathbf{3}$ & $\mathbf{5}$ & $\mathbf{1 0}$ & $\mathbf{1 5}$ & $\mathbf{2 0}$ \\
\hline $\mathrm{RB}$ & 6 & 15 & 25 & 50 & 75 & 100 \\
\hline
\end{tabular}

\subsection{Proposed Switch On/Off Algorithms}

As mentioned in Section 4.3.5, developing models for the proposed vision is an NPHard problem. Finding an optimal solution for the optimization problem in hand analytically becomes very difficult. By finding all the possible SINR maps for all combinations of Pico-eNBs before hand is a possible way to avoid solving the problem analytically. However, with the larger number of eNBs, the complexity of the problem increases, because the number of possible eNB combinations increases exponentially. For example, if a network consists of 20 Pico-eNBs, the number of possible combinations is $N_{\text {comb }}=2^{20}-1$ which is over 1 million possible combinations. As a result, a proper switch on/off algorithm that can reduce the decision time of the optimization problem needs to be implemented.

In this section, we discuss two switch on/off algorithms that were used to implement the optimization problem explained in Section 4.2. The purpose of the two 
algorithms is to minimize the TEPB of the network. The first algorithm uses a brute force method to achieve the best network setup for the given UE distribution, whereas the second algorithm uses greedy drop algorithm.

\subsubsection{The Centralized Switch On/Off Algorithm}

In this section, we propose a centralized switch on/off algorithm (CSA) to allow the EMS/NMS to control which Pico-eNB to switch on or off. The CSA was used to find the best network setup that meets these two requirements:

- Average Pico-eNB throughput greater than or equal to 5 Mbps.

- Network outage percentage less than $1 \%$.

It is important to note that these conditions can be changed by the network operator in order to favor certain objectives. The steps of the proposed eNB centralized switch on/off algorithm are shown in Figure 4.7 and explained below. It is important to note that the first three steps are all done in a pre-processing stage. By doing these calculations beforehand, the rest of the algorithm can run faster.

Step 1: The EMS/NMS divides the network into groups of Pico-eNBs as explained in Section 4.3. For example, if a network contains 74 Pico-eNBs, and the processing power of the EMS/NMS can run the algorithm for 37 Pico-eNBs with an acceptable time. Then the EMS/NMS divides the network into two groups of 37 neighboring Pico-eNBs and runs the remaining steps of the switch on/off algorithm for each group separately to find which Pico-eNB to switch on/off. More details on the process of dividing the network into groups of Pico-eNBs will follow in Section 4.4.1.2. 


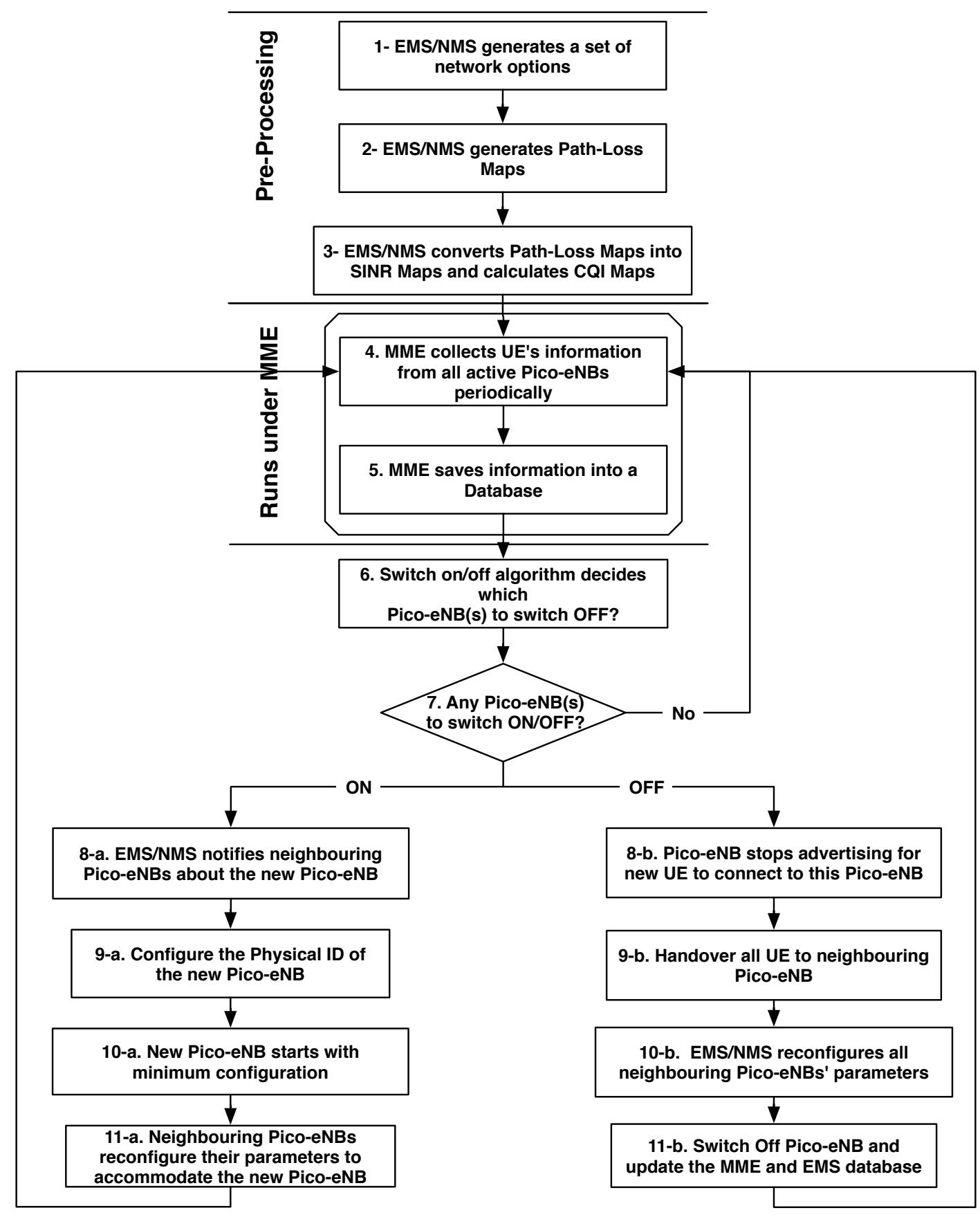

Figure 4.7: The centralized switch on/off algorithm

Step 2: The EMS/NMS generates the SINR map for all Pico-eNBs in the network that are under its control. The SINR map is generated using the propagation 
models explained in the previous section.

Step 3: The path-loss is converted into SINR using Equation 4.11). After calculating the SINR, it is converted into CQI using Table 4.1. These CQI values are saved in a database in the EMS/NMS and are updated periodically with information collected from UE and Pico-eNBs connected to the EMS/NMS.

Step 4: The MME collects UE status periodically (location, required data rate, and the CQI that they require) from all Pico-eNBs connected to it.

Step 5: The MME saves this information into a database so it can use it later to decide which Pico-eNB needs to be switched on or off.

Step 6: The EMS/NMS periodically runs the algorithm to determine if any changes need to be done to the network setup. If multiple solutions meet the conditions that are required to decide which combination of Pico-eNBs is best at that time, the algorithm will go towards the combination that requires the least number of UE handover to maintain the QoS.

Step 7: Any Pico-eNBs need to be switched on/off? If yes, then continue to on/off Steps 8 to 11, if not, go back to Step 4. It is possible for the CSA to switch on/off multiple Pico-eNBs at the same time.

\section{Switch ON:}

Step 8-a: If the algorithm decides that a Pico-eNB needs to be switched on, then the EMS/NMS notifies neighboring Pico-eNBs about the new Pico-eNB.

Step 9-a: The EMS/NMS assigns a Physical ID of the new Pico-eNB.

Step 10-a: The new Pico-eNB starts with minimum transmit power level and antenna tilt then starts increasing them, until reaching the desired coverage area. 
Step 11-a: Neighboring Pico-eNBs reconfigure their parameters (transmit power, coverage area, and antenna tilt) to accommodate the new Pico-eNB. The PicoeNB is up and running and ready for UE to connect to it.

\section{Switch OFF:}

Step 8-b: If the algorithm decides that a Pico-eNB needs to be switched off, then the Pico-eNB stops advertising for new UE to connect to it.

Step 9-b: The Pico-eNB starts the handover procedure for all UE to neighboring sites to avoid dropping any UE and maintain QoS. In some cases, there might be some dropped UE, which is acceptable as long as the network outage constraint is not exceeded.

Step 10-b: After all UE are moved to neighboring Pico-eNBs, the EMS/NMS reconfigures the neighboring Pico-eNBs power, to cover the outage from switching off that Pico-eNB.

Step 11-b: The Pico-eNB is switched off and the MME and EMS/NMS databases are updated.

\subsubsection{Switch On/Off Example}

In this section, a switch off example will be explained. Figure 4.8 shows a network that consists of 7 Pico-eNBs at the centre with an inter-site distance of $350 \mathrm{~m}$. These 7 Pico-eNBs illustrate the Pico-eNBs in the network under study, which are surrounded by 12 Pico-eNBs representing the neighboring networks. The white area surrounding each Pico-eNB represents its coverage and is separated by the colored lines. These

lines are not smooth because of the randomness caused by the shadowing $\left(F_{s}\right)$ as detailed in Section 4.3.1. 


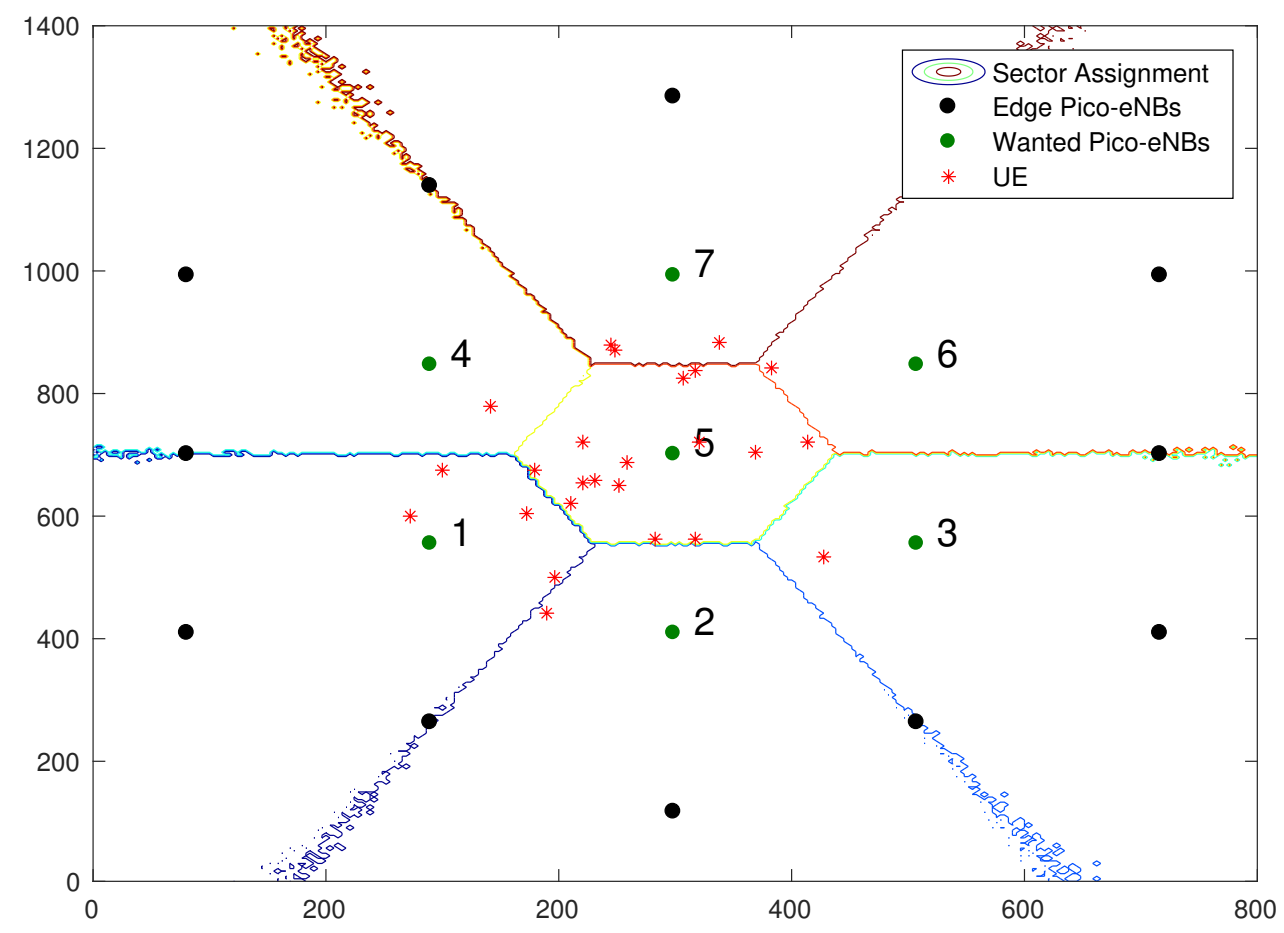

Figure 4.8: Initial network

Figure 4.8 also shows that Pico-eNBs $(2,3,4$, and 6) have only one UE connected to them; hence the EMS/NMS will run the centralized switch on/off algorithm to calculate the feasibility of switching on/off one (or more) of these Pico-eNBs. The algorithm runs steps 1 through 5 from Figure 4.7 to decide if a Pico-eNB needs to be switched off. Then, the procedure to switch off a Pico-eNB is executed, as shown in steps 8-b through 11-b in Figure 4.7. The CSA checks all the possible combinations of the 7 Pico-eNBs $\left(N_{\text {comb }}=2^{7}-1=127\right)$ to be turned on/off and finds that the best network setup that fulfills the requirements is to switch only Pico-eNB (6) off.

The new network setup, after running the centralized switch on/off algorithm and switching off Pico-eNB (6), is shown in Figure 4.9. As we can see, neighboring PicoeNBs (i.e. 3, 5 and 7) are now covering (as much as possible) the area left uncovered 


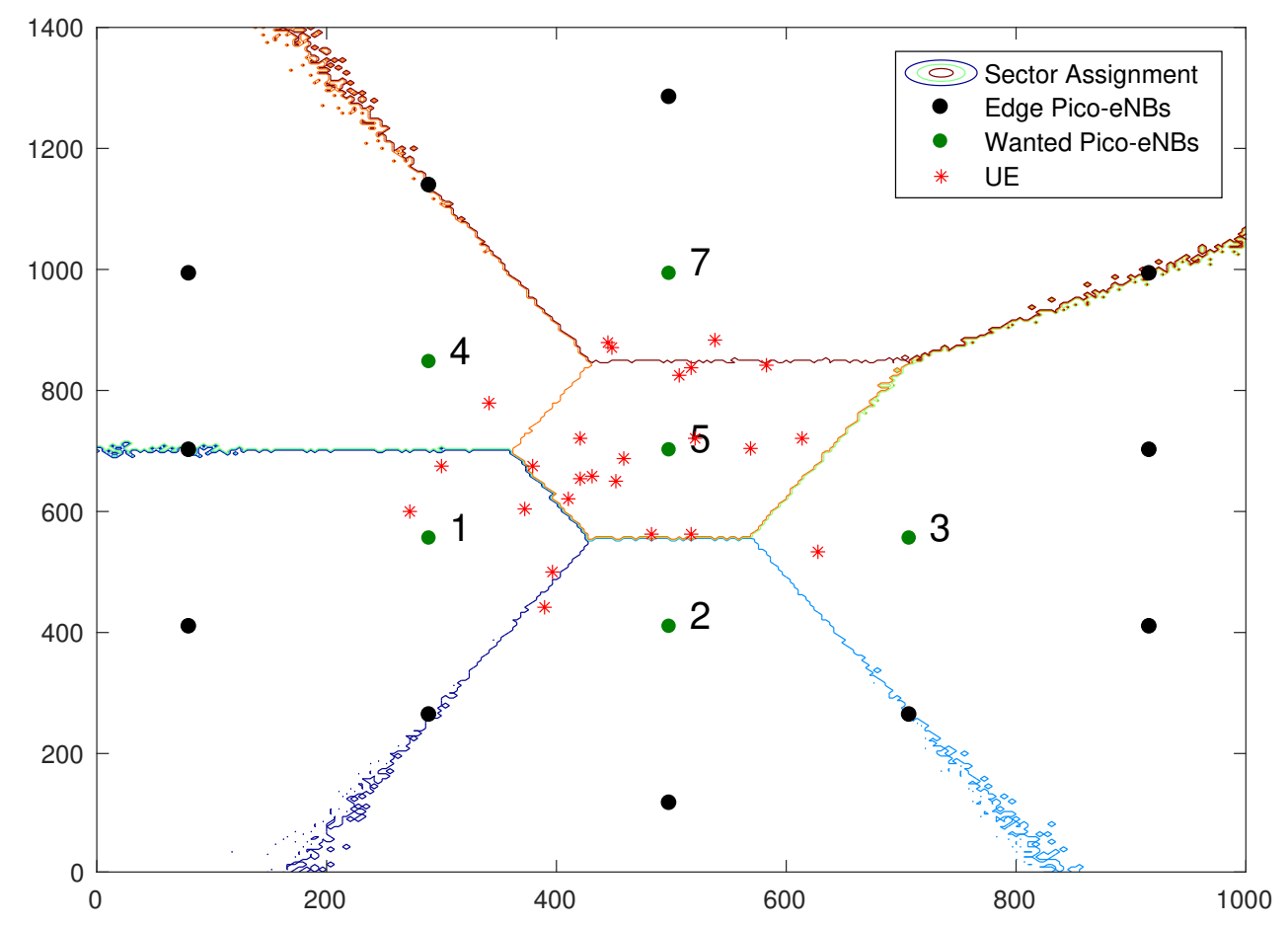

Figure 4.9: Network after centralized switch on/off algorithm is applied and PicoeNB (6) is switched off

from Pico-eNB (6). The same procedure for switching on a Pico-eNB (steps 8-b through 11-b in Figure 4.7 can be used for adding a new Pico-eNB to the network.

\subsubsection{Implementing the CSA in a Bigger Network}

The CSA has a processing time limitation. When the number of Pico-eNBs in the network increases, the number of possible combinations increases exponentially $\left(N_{c o m b}=2^{N}-1\right)$. Therefore, a work around that problem is needed. In order to reduce the decision time for the CSA, the network is divided into smaller intersecting clusters of eNBs. Assuming a triangular lattice is used in the initial network setup, the network can be divided into clusters of 7 eNBs (i.e. $N_{\text {comb }}=2^{7}-1=127$ ). Next, the CSA evaluates each cluster to decide which Pico-eNB to switch on/off, by 
finding all the possible combinations of each cluster. Finally, the CSA decides which Pico-eNBs to switch on/off for all clusters, but there might be multiple decisions (on or off) for each of the intersecting Pico-eNBs. If 4 out of 7 of these decisions are to switch off the eNB, that eNB will be turned off, otherwise being left on.

For example, let us assume we have a network with 37 Pico-eNBs surrounded by 24 edge Pico-eNBs as shown in Figure 4.10 and 4.11. The CSA divides the network into intersecting groups of 7 Pico-eNBs. If we consider Pico-eNB (21) as an example, in order for the simulator to decide if that Pico-eNB needs to be switched off, the CSA is executed for the 7 groups that Pico-eNB (21) falls into (groups 1 to 7 in Figures 4.10 and 4.11). In this example, let us assume that the output of the CSA for groups 1 to 3 is to keep Pico-eNB (21) on while the output for groups 4 to 7, is to switch it off. Since the majority agrees to switch off, then the algorithm goes into the switch off process. If the decisions were reversed ( 4 on and 3 off), then Pico-eNB (21) would remain on or it will be turned on if it was already off. The same decision process will be applied to all Pico-eNBs in the network.

\subsubsection{The Enhanced Centralized Switch On/Off Algorithm}

The previous algorithm has a processing time limitation. Table 4.3 shows the time required by the CSA to decide which Pico-eNB(s) to switch on/off for different numbers of Pico-eNBs. The main cause for the time limitation is that the CSA has to find decisions for all the possible network combinations $\left(N_{\text {comb }}=2^{N}-1\right)$ in order to find which Pico-eNB to switch on/off. Table 4.3 also shows that even if the workaround proposed in the previous section slightly alleviates the problem, it also deteriorates the quality of the solution.

To solve this issue, an enhanced switch on/off algorithm (ECSA) is proposed. The algorithm starts by assuming that UE location is fixed then switches off one PicoeNB at a time until it reaches the network's steady state. Then, the algorithm starts 


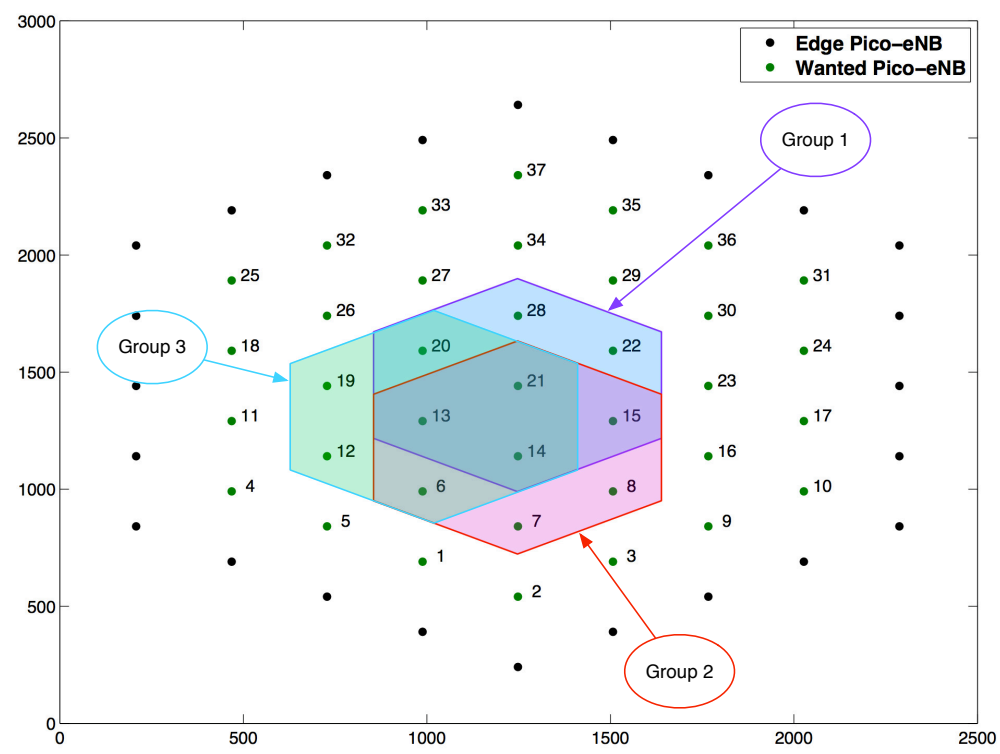

Figure 4.10: CSA Pico-eNB grouping example (groups 1 to 3 )

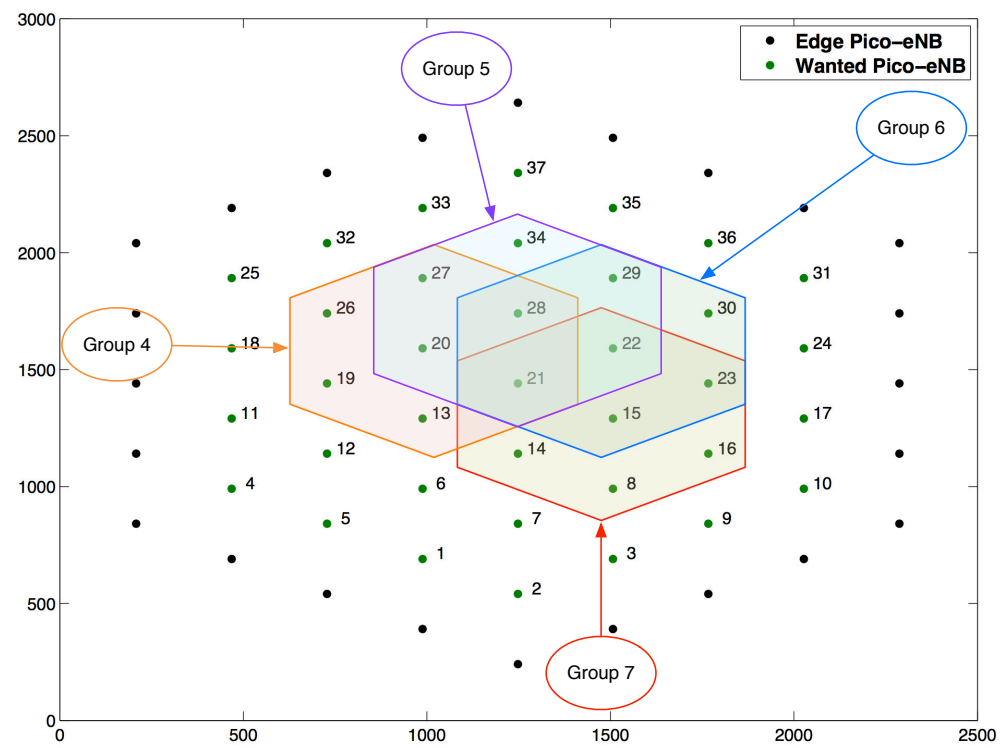

Figure 4.11: CSA Pico-eNB grouping example (groups 4 to 7 ) 
Table 4.3: Processing time of the CSA for 500 - 1500 UE in the network

\begin{tabular}{lccccc}
\hline No. of Rings & $\mathbf{1}$ & $\mathbf{2}$ & $\mathbf{3}$ & $\mathbf{4}$ & $\ldots$ \\
\hline $\begin{array}{l}\text { No. of Pico-eNBs } \\
\text { No. of Combinations }\end{array}$ & 7 & 19 & 37 & 61 & $\ldots$ \\
$\begin{array}{l}\text { No. of Clusters of } \\
7 \text { Pico-eNBs }\end{array}$ & 1 & 524288 & $1.37 \times 10^{11}$ & $2.8 \times 10^{18}$ & $\ldots$ \\
$\begin{array}{l}\text { No. of Possible Clus- } \\
\text { ters }\end{array}$ & 1 & 133 & 37 & 61 & $\ldots$ \\
$\begin{array}{l}\text { Time for CSA to get } \\
\text { a decision }\end{array}$ & $3.5-7$ sec & $8-16$ min & $15-30$ min & $25-50$ min & $\ldots$ \\
\hline
\end{tabular}

switching on/off Pico-eNBs to follow the movement of UE during the day. The ECSA steps are shown in Figure 4.12 and explained below. The main difference between CSA and ECSA is in the process of finding which Pico-eNB to switch on/off which starts at step 6 in the algorithm. Steps 1 to 5 are identical in both algorithms.

Step 6: The EMS/NMS periodically runs the algorithm to determine if any changes need to be done to the network setup. Is there a Pico-eNB to switch on/off? If yes, go to step 7 , if not go back to step 4 .

Switch OFF: The enhanced switch on/off algorithm finds which Pico-eNB meets the requirements to switch off. These requirements are:

- Pico-eNB has the lowest throughput which makes it easier to handover UE connected to that Pico-eNB to neighboring Pico-eNBs.

- Number of user affected by the switch off should be kept to a minimum. In our case, we considered $10 \%$ although other values can be considered.

- Network outage caused by switching off that Pico-eNB is less than or equal to $1 \%$. Overall network outage should be minimal which is important to 


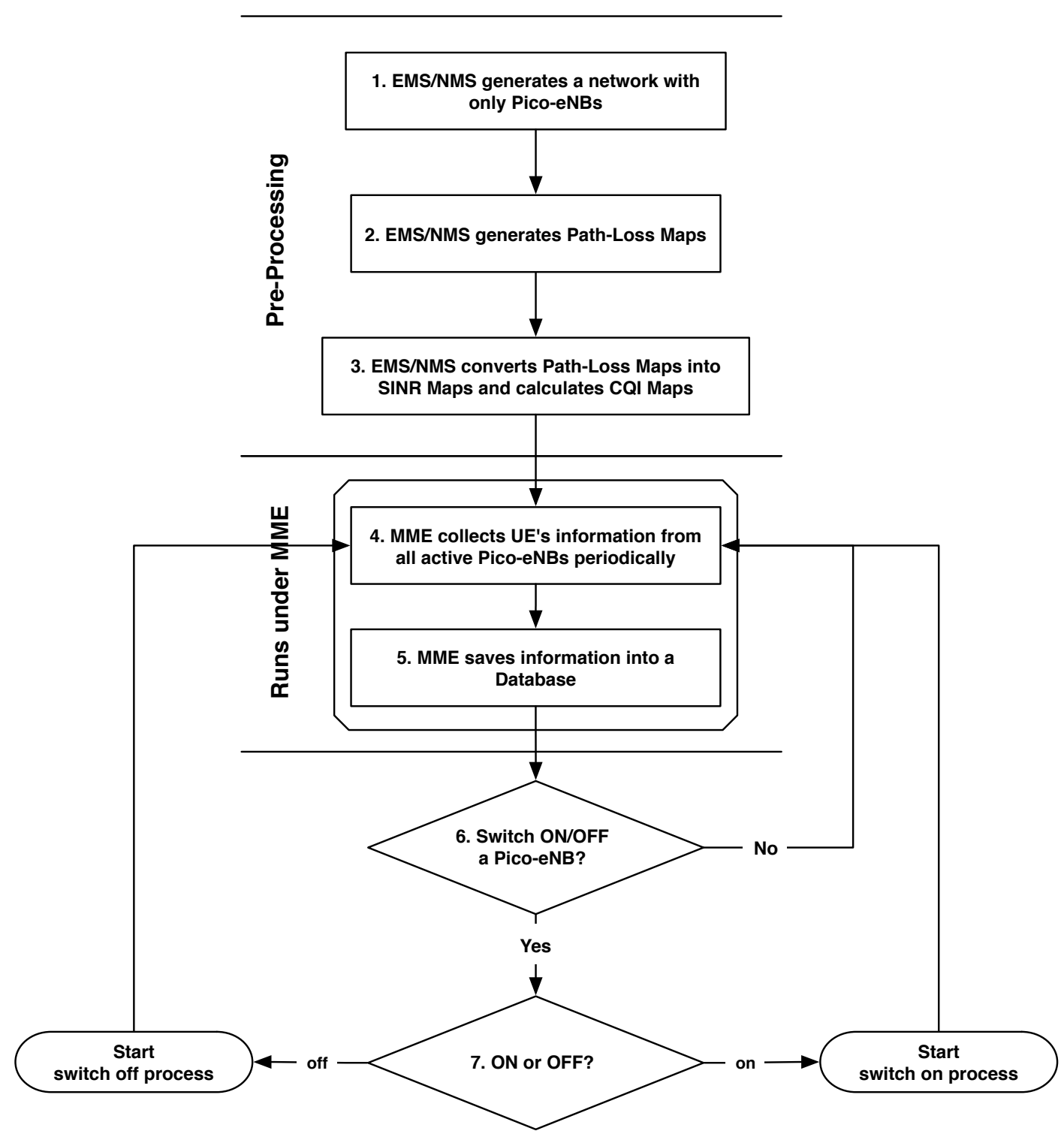

Figure 4.12: The enhanced centralized switch on/off algorithm

maintain the desired QoS.

It is important to note that these values can be changed by the network provider 
so that different objectives can be met. For example, if no outage are permitted, then the value for the network outage can be set to 0 . However, it is important to remember that our goal is to achieve a tradeoff between the throughput and the energy consumed. So even if switching off a Pico-eNB might cause a slight degradation in the throughput of the network, the saved energy might still improve the value of the TEPB. If multiple Pico-eNBs meet the conditions that are required to decide which Pico-eNB to switch off, the algorithm selects the Pico-eNB that minimizes the TEPB.

Switch ON: In order for the enhanced switch on/off algorithm to find which PicoeNB to switch on, it searches for a switched off Pico-eNB that is closest to the Pico-eNB with the highest traffic load. If the algorithm finds a switched off Pico-eNB in that area, the algorithm goes to the next step.

Step 7: After the enhanced switch on/off algorithm decides which Pico-eNB to switch on or off, the switch on/off process will begin. If a Pico-eNB needs to be switched off, then it stops accepting new UE and hands over the UE to neighboring Pico-eNBs and switches off. The EMS/NMS then reconfigures neighboring Pico-eNBs to accommodate the outage of switching off that Pico-eNB. On the other hand, when a Pico-eNB needs to be switched on, the EMS/NMS notifies neighboring Pico-eNBs about the new Pico-eNB, which starts with minimum power level then starts increasing it at the same time, neighboring Pico-eNBs reconfigure their parameters to accommodate the new Pico-eNB. The switch on/off processes were discussed in more details in Section 4.4.1.

As the process of finding which Pico-eNB to be switched on/off takes some time, and in order to reduce the overhead, the algorithm runs periodically in the background in the EMS/NMS. Hence, it is impossible to create a system that can change at the same rate as UE change. The ECSA uses the statistical information saved by the 
MME in step 5 to generate a snapshot of UE locations at a specific time, which is used in the decision of switching on/off Pico-eNBs. Therefore, the real time change in UE location, direction, or speed does not affect the decision of the ECSA.

\subsection{UE Distribution and Mobility Model}

In this section, the mobility pattern used in simulations to evaluate both algorithms (CSA and ECSA) is discussed. One of the most important issues to take into account when creating a simulation environment in LTE networks is to correctly model the behavior of UE movement. The main problem of modeling the behavior of UE belonging to a mobile network is the lack of a unique or straightforward solution.

Although the switch on/off algorithm should not rely on the type of UE distribution, three different types of UE distributions were used to enforce that point. These distributions were Gaussian, uniform and Manhattan UE distributions. For Gaussian and uniform UE distributions, every time the UE are expected to move, new UE locations are generated (using these distributions), while in the Manhattan model, the UE mobility is based on speed and direction change as will be explained in more details in the following sections.

\subsubsection{Gaussian Distribution}

The Gaussian distribution was used in some simulations to test the effect of UE concentration at some locations in the network as the random distribution is bell-shaped. The probability density function of the Gaussian distribution can be calculated by Equation 4.28.

$$
f\left(x \mid \mu, \sigma^{2}\right)=\frac{1}{\sqrt{2 \sigma^{2} \pi}} e^{-\frac{(x-\mu)^{2}}{2 \sigma^{2}}}
$$

Where: 
- $\mu$ is the mean and center of the distribution.

- $\sigma$ is the standard deviation of the distribution.

- $\sigma^{2}$ is the variance of the distribution.

\subsubsection{Uniform Distribution}

Another practical UE distribution, is the uniform distribution. This distribution can be used to simulate UE movement during the day, when UE are randomly distributed over the network with no specific points of concentration. Also, UE can be distributed with no constraint other than that it is contained in the distribution's support range (network area), as shown in Equation 4.29).

$$
f(x)= \begin{cases}\frac{1}{\max -\min } & \text { for } x \in[\text { min, } \max ] \\ 0 & \text { otherwise }\end{cases}
$$

Where:

- $\min$ and $\max$, are distribution minimum and maximum values.

\subsubsection{Manhattan Mobility Model}

The Manhattan model was used to create urban mobility scenarios [89]. The simulation area is divided into uniform block sizes with two-way streets (one lane in each direction) as shown in Figure 4.13. The darker squares represent the downtown area, which has a different speed range compared to the surrounding areas (white squares).

A random mobility model for UE movement in two dimensions was used. The speed $(v)$, acceleration $(a)$ and direction $(\theta)$ changes are probabilistic. Two stochastic processes were used to determine when the speed and direction of a UE will change 90$]$. 


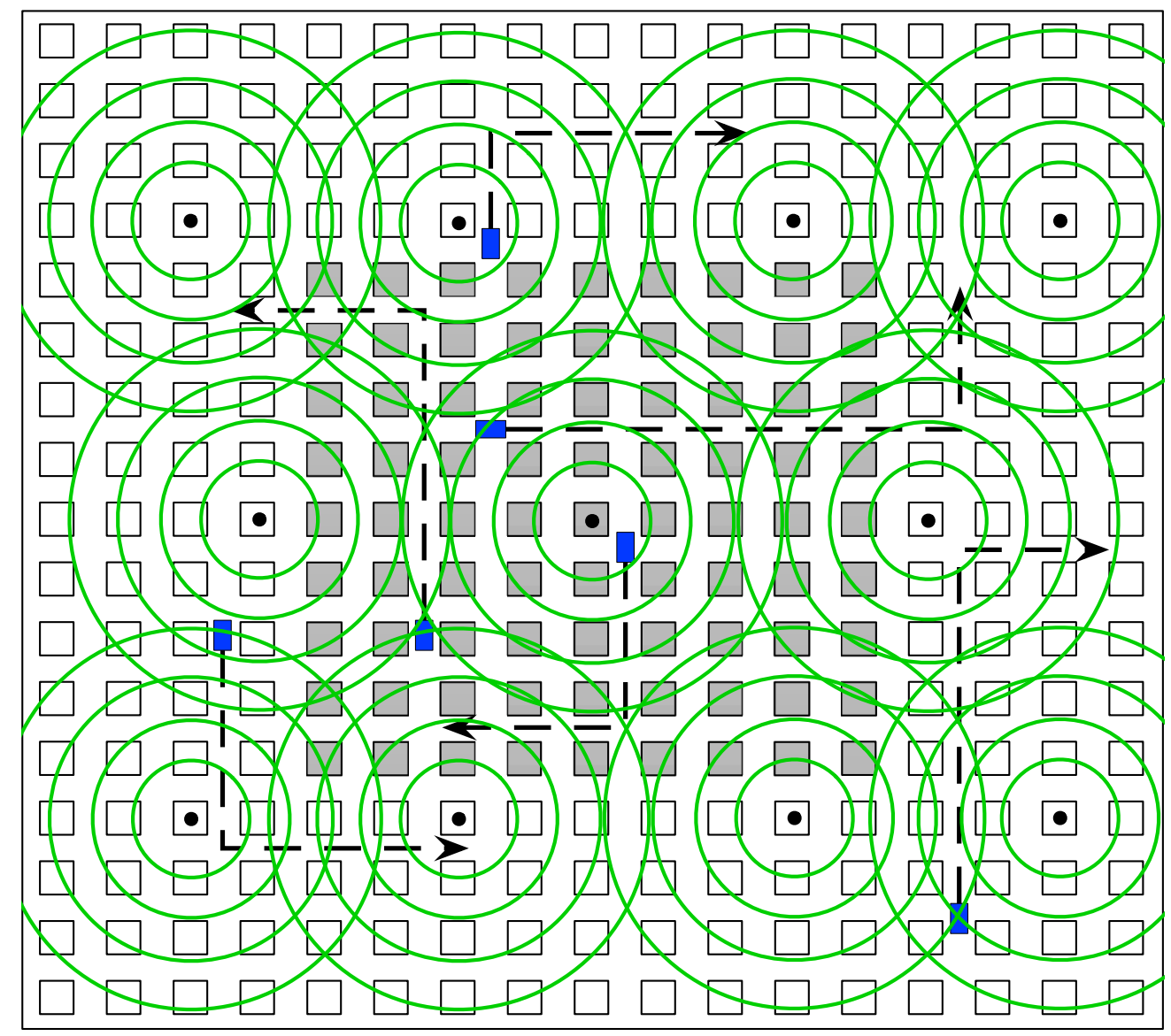

Figure 4.13: Network setup and UE movement

\subsubsection{Speed Change}

In order to model the speed behavior of UE, target speeds and acceleration were assigned for each UE. The UE starts with an initial constant speed $v$ until a new random target speed is chosen. The UE accelerates or decelerates until it reaches the new desired speed. Each UE at time $t$ has three defined parameters:

- current speed $v(t)$ in $m / s$.

- current acceleration $a(t)$ in $m / s^{2}$.

- target speed $v_{\text {new }}(t)$ in $\mathrm{m} / \mathrm{s}$. 
In addition, each UE is defined by three static speed parameters, whose value depends on whether they fall in the downtown or surrounding area:

- maximum speed $v_{\max }$.

- set of preferred speeds $\left\{v_{\text {pref } 0}, v_{\text {pref } 1}, v_{\text {pref } 2}, \ldots\right\}$.

- maximum and minimum values for acceleration.

\subsubsection{Direction Change}

The concept of direction is similar to the speed change. Each UE has an initial direction $\theta(t=0)$ chosen from a uniform distribution, and the same distribution will be used to choose a new direction for each UE at time $t^{*}$, as shown in Equation (4.30).

$$
p(\theta)=\frac{1}{\pi} ; \quad 0 \leq \theta<\pi
$$




\section{Chapter 5}

\section{Simulation Results and Analysis}

In this chapter, we explain in details the system-level simulator developed to generate the results used to evaluate the CSA and the ECSA algorithms. Then, a comparison between the two algorithms is simulated and the results are examined. Finally, the ECSA is compared with a similar switch on/off algorithm from the literature.

\subsection{System-Level Simulator}

To ensure accurate results when testing, applying and comparing different algorithms, a realistic simulation platform is needed. In Chapter 4, we discussed the details on how to model the wireless channel between the eNB and the UE. We designed a system-level simulator that evaluates the performance of the downlink of an LTE network based on the guidelines given by the 3GPP group [1].

There are several LTE network simulators available online but one stands out above all other simulators: Vienna LTE system level simulator [91], designed in MATLAB. The main purpose of said simulator is to evaluate the network performance for new cell planning and scheduling algorithms. Simulating every aspect of the link between the UE and eNB (as they did) is impractical for switch on/off algorithms due to the vast amount of computational power required. Thus, we designed a system-level 
simulator that uses similar procedures to simulate an LTE network, while replacing some of the functions that were unrelated to our field of study with simplified models that capture the essential features of these functions with high accuracy and low complexity.

Our simulator outputs trace files containing throughput, network CQI, network outage, number of eNBs on/off, locations of UE and TEPB, as figures of merit. We defined a region of interest (ROI) in our simulation in which eNBs and UE are positioned. The UE movement and transmission of the downlink is only simulated within the ROI. The simulation length was measured in transmission time interval (TTI), where every TTI equals 1 msec. Given the number of rings $\left(N_{r}\right)$ in the network, the total number of eNBs $\left(N_{e N B s}\right)$ can be calculated with Equation (5.1). The simulator flow follows the pseudo-code shown in Algorithm 1. To validate the performance of our system-level simulator, different modules were tested and compared with Vienna's simulator to insure correct results are achieved, such as UE to eNB allocation, UE scheduling, network throughput and SINR map calculation. Furthermore, multiple network scenarios with 1 and 2 rings of Macro-eNBs at the centre of the network and 100 and 500 UE uniformly distributed were simulated in both Vienna's and our simulator. The results show that both simulators behave similarly.

$$
N_{e N B s}=\left(\sum_{i=1}^{N_{r}}(i \times 6)\right)+1
$$

In Chapter 4, we introduced two switch on/off algorithms (CSA and ECSA). The process of finding which eNB to switch on/off depends on the type of algorithm used in the simulation. The simulation of these algorithms is discussed in the two following subsections. 


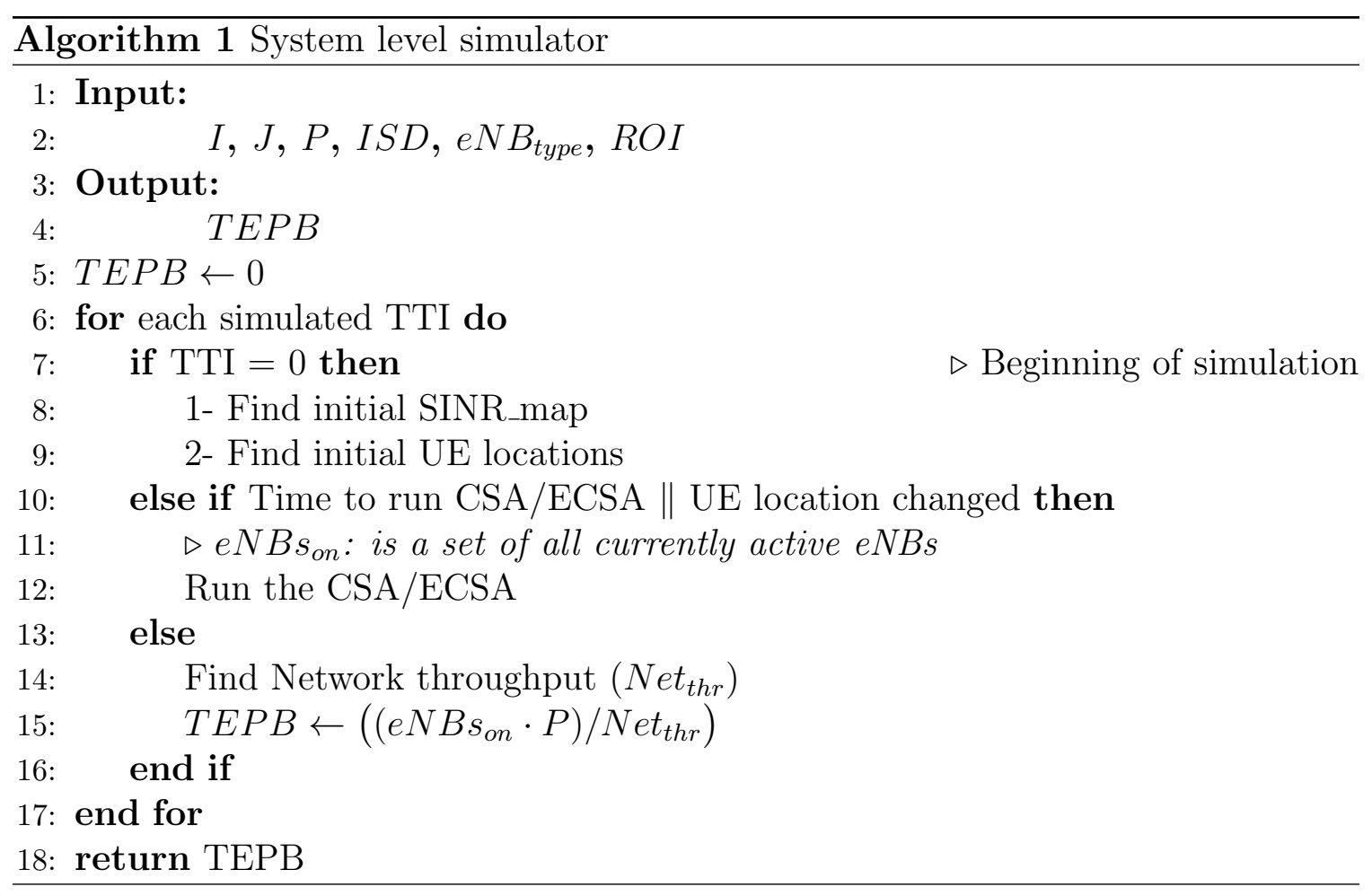

\subsubsection{CSA Simulation}

As explained in Section 4.4.1, with the increase in the number of eNBs in the system, the CSA has a processing time limitation. In order to reduce the run time for the CSA, the network was divided into smaller intersecting clusters of eNBs. The CSA then evaluates each cluster to decide which eNB(s) to switch on/off by finding all possible combinations of each cluster. If 4 out of 7 of these decisions are to switch off the eNB, then that eNB will be turned off, otherwise it will remain on. The same decision process will be applied to all eNBs in the network. For more details, refer to Section 4.4.1.2,

\subsubsection{ECSA Simulation}

In this section, the ECSA part of our system-level simulator is explained. We will assume the same network setup as in Figure 4.10, In order for the simulator to find 
which Pico-eNB to switch on/off using the ECSA, the simulator starts with all PicoeNBs on and UE in initial locations. The simulator runs the ECSA to calculate the feasibility of switching on/off a Pico-eNB that matches the requirements discussed in Section 4.4. Then, the simulator switches off one Pico-eNB at a time until it reaches the steady state network setup, which means that no more Pico-eNBs can be switched off for that specific UE distribution. This step can be done by the network operators at the time that they have the lowest traffic, in order to get a good starting point for when the UE number/location start to change. After the ECSA reaches the steady state network setup, it starts switching on/off Pico-eNBs based on the movement of UE. The UE locations start to change based on the type of mobility model chosen. The simulator then takes a snap shot of the new UE locations periodically (every $5 \sec (5000$ TTI $)$ ) and runs the ECSA to switch on/off a Pico-eNB at a time based on these new locations.

\subsection{CSA Simulation Results and Analysis}

\subsubsection{Simulation Setup}

A network layout with Pico-eNBs is simulated using the system-level simulator explained in the previous section. As shown in Figure 5.1, the simulated network consists of 37 Pico-eNBs with an inter-site distance (ISD) of $350 \mathrm{~m}$, and these Pico-eNBs are surrounded with 24 Pico-eNBs to simulate the edge effect of the network.

The network topology consists of a standard set of low power Pico-eNBs with omni-directional antennas. The CSA can be applied for any UE distribution. To test the performance of the CSA, UE are assumed to be Gaussian distributed with a standard deviation $\sigma=125$ and mean $(\mu)$ which is equal to the centre of the UE distribution. We assume that the base station antenna height is fixed at $15 \mathrm{~m}$ 


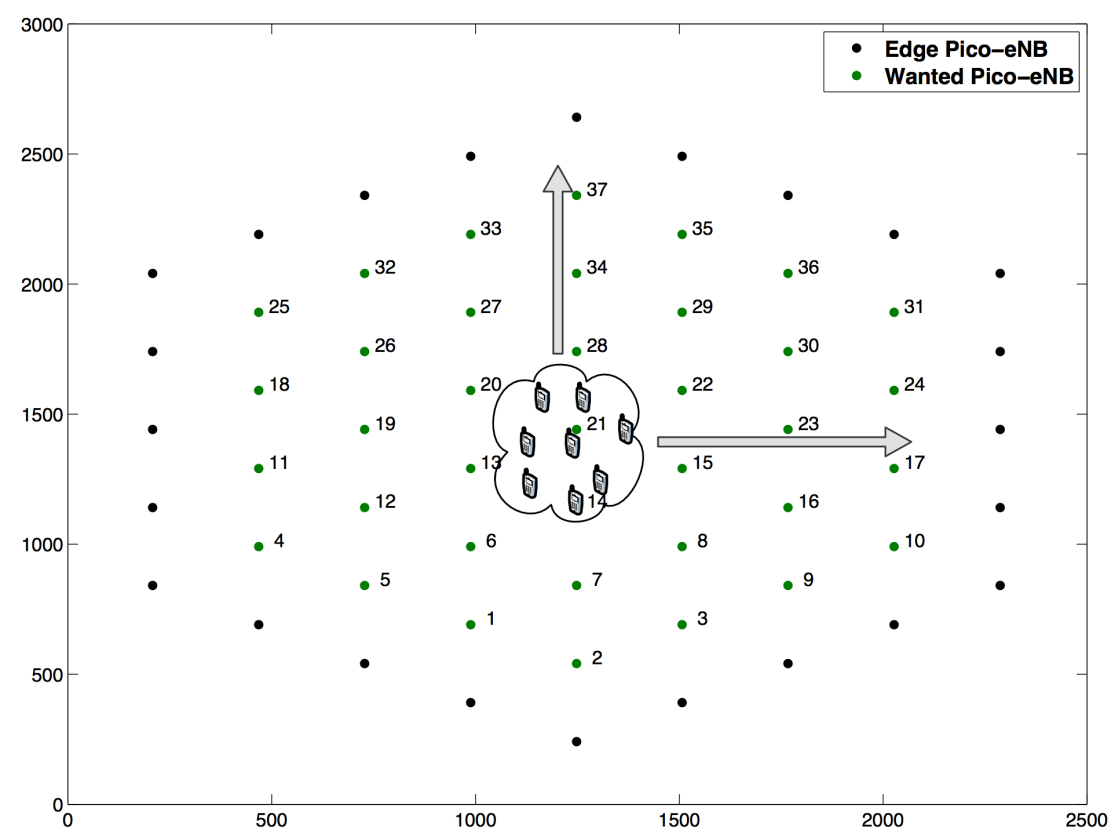

Figure 5.1: Simulation setup to calculate the effect of distance between the centre of the network and UE's grouping

above the rooftop, and a carrier frequency of $2 \mathrm{GHz}$ is used. The default simulation parameters are summarized in Table 5.1. The throughput per eNB (in Mbps) can be calculated with Equation (5.2).

\subsubsection{Evaluation of the Range Effect on the CSA}

To simulate UE distribution movement throughout the day, the simulation is repeated for different UE distribution centers $(\mu)$ moving from the centre to the edges of the network, as shown in Figure 5.1. The reason for choosing those two UE's movement directions is to evaluate the effect of UE movement from one network cluster to the neighboring network cluster. 
Table 5.1: Simulation parameters to evaluate the CSA

\begin{tabular}{lc}
\hline Parameter & Setting \\
\hline Macro-eNB ISD & $900 \mathrm{~m}$ \\
Pico-eNB ISD & $350 \mathrm{~m}$ \\
No. of Pico-eNBs & 37 \\
No. of Combinations per Group & 127 \\
No. of UE & 500 \\
No. of RBs per UE & 10 \\
Pico-eNB Tx Power & $36 \mathrm{dBm}$ \\
Macro-eNB Tx Power & $46 \mathrm{dBm}$ \\
Thermal Noise & $-174 \mathrm{dBm} / \mathrm{Hz}$ \\
\hline & Gaussian distribution $\sigma=125$ \\
UE Distribution & at the centre of the network \\
\hline Bandwidth & 20 MHz at 2 GHz carrier frequency \\
Antenna Height & $15 \mathrm{~m}$ \\
Traffic Model & Full buffer, full load
\end{tabular}

\subsubsection{Methodology for the Evaluation of the Range Effect}

In order to evaluate the performance of our model, 3 different scenarios are evaluated. The first scenario simulates a network having only Macro-eNBs. A Macro-eNB with an ISD of $900 \mathrm{~m}$ is placed at the centre and surrounded with 7 Macro-eNBs to simulate the edge effect. This scenario will provide the baseline performance for Macro-eNB networks.

In the second scenario, the simulation is run the same way as in scenario 1 except that the Macro-eNB is replaced with 3 rings of Pico-eNBs (as shown in Figure 5.1) with an ISD of $350 \mathrm{~m}$. In this scenario, all Pico-eNBs are turned on. 
Finally, the third scenario is using the same network setup as in the second scenario but this time, the proposed CSA is used. The EMS/NMS decides which Pico-eNB will turn on/off to fulfill the requirements discussed in Section 4.4. The algorithm will run in the background of the EMS/NMS to generate the network setup that best meets the requirements. The algorithm can be triggered by the network operator every 30 minutes to minimize the TEPB of the network.

The UE's distribution centre changes every 5 sec $(5,000$ TTI). The simulation was repeated 10 times because each simulation represents a UE distribution at a different location in the network and $73 \mathrm{UE}$ center of distribution locations were used (36 moving to the right edge and 37 moving to the upper edge). The simulation was used produce the results which presents the effect of changing the range between the UE and centre of the network on TEPB.

The SINR maps for all Pico-eNBs combinations and with only one Macro-eNB are generated. Because frequency and network area in this thesis are constants, to simplify the results, the TEPB unit was changed from Watts $/$ bps $/ \mathrm{Hz} / \mathrm{m}^{2}$ (Equation 4.1) to Joules/bit. Using Equation (5.2), the algorithm calculates the throughput for every UE, then it will calculate the average network throughput.

$$
\text { Throughput(bps) }=T B S \cdot N_{T B} \cdot S f p s
$$

Where:

- $T B S$ is the Transport Block Size (bits) / Subframe

- $N_{T B}$ is the Number of Transport Blocks / Subframe

- Sfps $=1000$ Subframes / Sec 


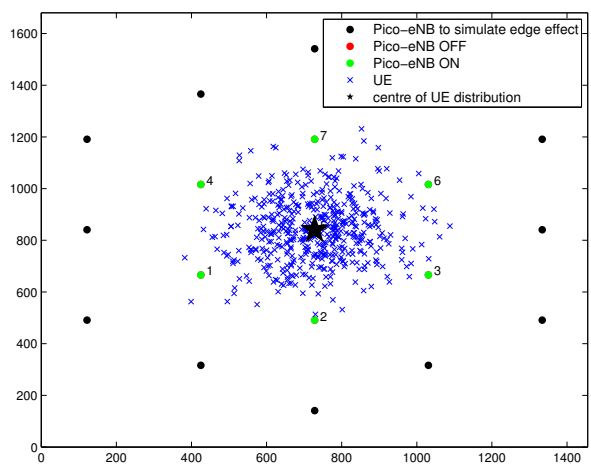

(a) Distance from centre $=0 \mathrm{~m}$

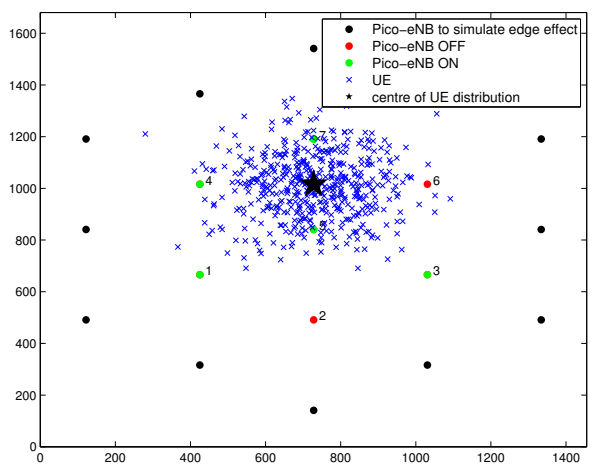

(c) Distance from centre $=140 \mathrm{~m}$

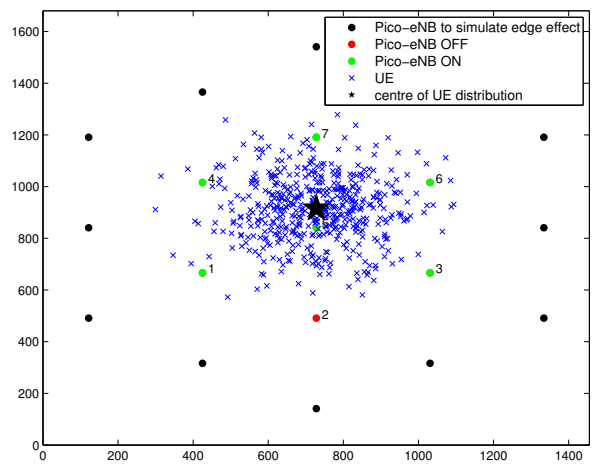

(b) Distance from centre $=70 \mathrm{~m}$



(d) Distance from centre $=280 \mathrm{~m}$

Figure 5.2: Sample simulation to simulate 500 UE moving from centre to the upper edge of the network

\subsubsection{Results for the Evaluation of the Range Effect}

In this section, an example is given to clarify the process that the CSA follows to find which Pico-eNBs to switch on/off which will help in understanding the simulation results discussed after. As explained in Section 5.1.1, to reduce the processing time of the CSA, the whole network is divided into clusters of 7 Pico-eNBs. In Figure 5.2 , UE are moving towards the upper edge of the network and the CSA decides which Pico-eNBs to switch on/off. When the UE distribution centre $(\mu)$ starts at the centre of the network, as shown in Figure 5.2a, the CSA finds that all Pico-eNBs in that cluster need to be switched on. When the UE start moving away from the centre of 
the network ( $\mu$ distance at $70 \mathrm{~m}$ from network centre), the CSA finds that Pico-eNB (2) needs to be switched off. Then, as the UE keep moving from the centre to the upper edge of the Pico-eNB cluster, in Figure 5.2c, the CSA finds that Pico-eNBs (2) and (6) need to be switched off. When the UE reach the edge of the Pico-eNB cluster shown in Figure $5.2 \mathrm{c}$, the CSA considers the UE entering the next Pico-eNB cluster as shown in Figure $5.2 \mathrm{~d}$ ( $\mu$ distance at $210 \mathrm{~m}$ from network centre). The CSA runs and finds that Pico-eNB (5) in that cluster needs to be turned off. The same procedure can be applied to UE distribution moving in any direction.

Keeping in mind the concept explained in the above example, the simulation results follow. When running the simulation with only one Macro-eNB at the centre of the network, the TEPB starts at $15.14 \times 10^{-12}$ Joules/bit, as shown in Figures 5.3 and 5.4. This is actually the lowest TEPB that can be achieved as users are very close to the Macro-eNB located at the centre. As users are moving away from the centre of the network, the average network throughput starts to decrease to zero when it reaches out of the range of the Macro-eNB which leads to an increase in the TEPB towards infinity. On the other hand, when replacing the Macro-eNB with 37 Pico-eNBs to cover the same area, the TEPB fluctuates between $1.45 \times 10^{-12}$ and $2.2785 \times 10^{-12}$ Joules/bit when users are moving towards the upper edge of the network, and between $1.43 \times 10^{-12}$ and $3.23 \times 10^{-12}$ Joules/bit when users are moving towards the right edge of the network. The fluctuation in TEPB happens as UE move away from the centre of the network. It is observed that the TEPB increases as UE move away from the centre of the network but when the UE get closer to a nearby Pico-eNB, the TEPB starts to decrease again. That is due to the decrease in the throughput as the UE move away from the centre of the network. The simulation results presented in Figures 5.3 and 5.4, show the effectiveness of replacing a Macro-eNB with a large number of Pico-eNBs. By using several PicoeNB, the TEPB remains relatively constant since the distance between the user and 


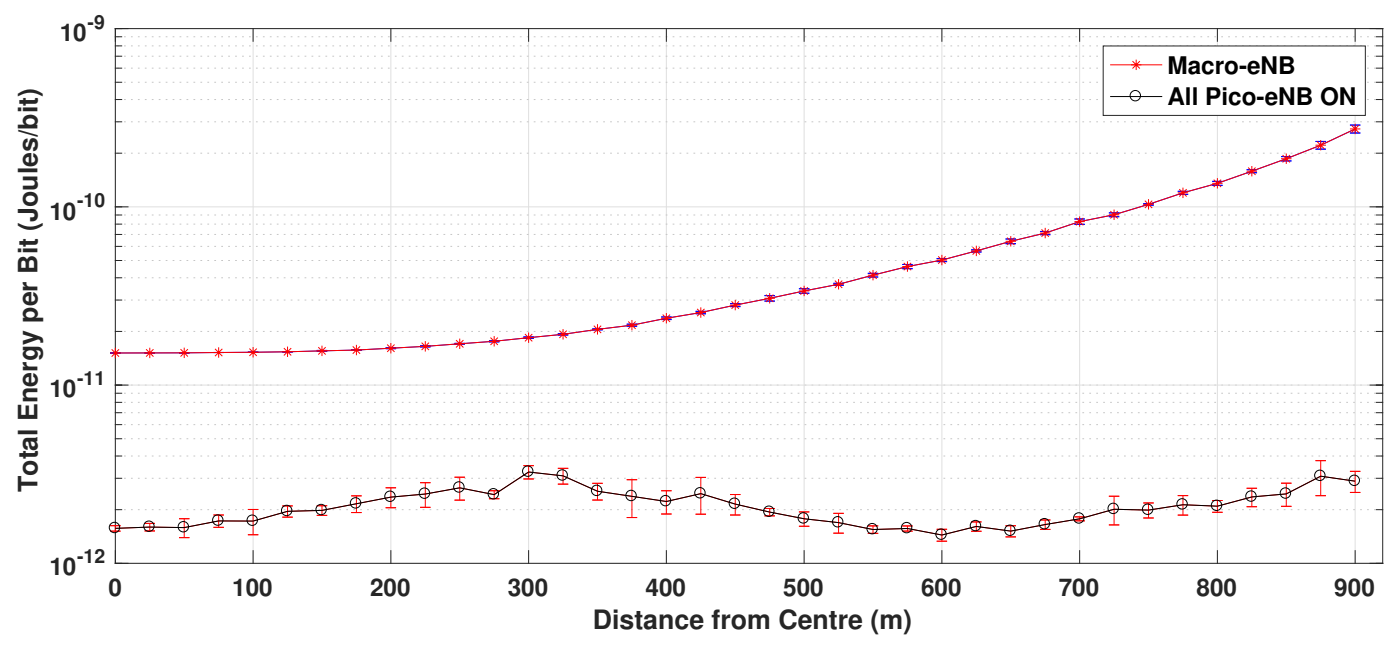

Figure 5.3: TEPB of Macro-eNB vs all Pico-eNBs on for UE moving from centre to the upper edge of the network

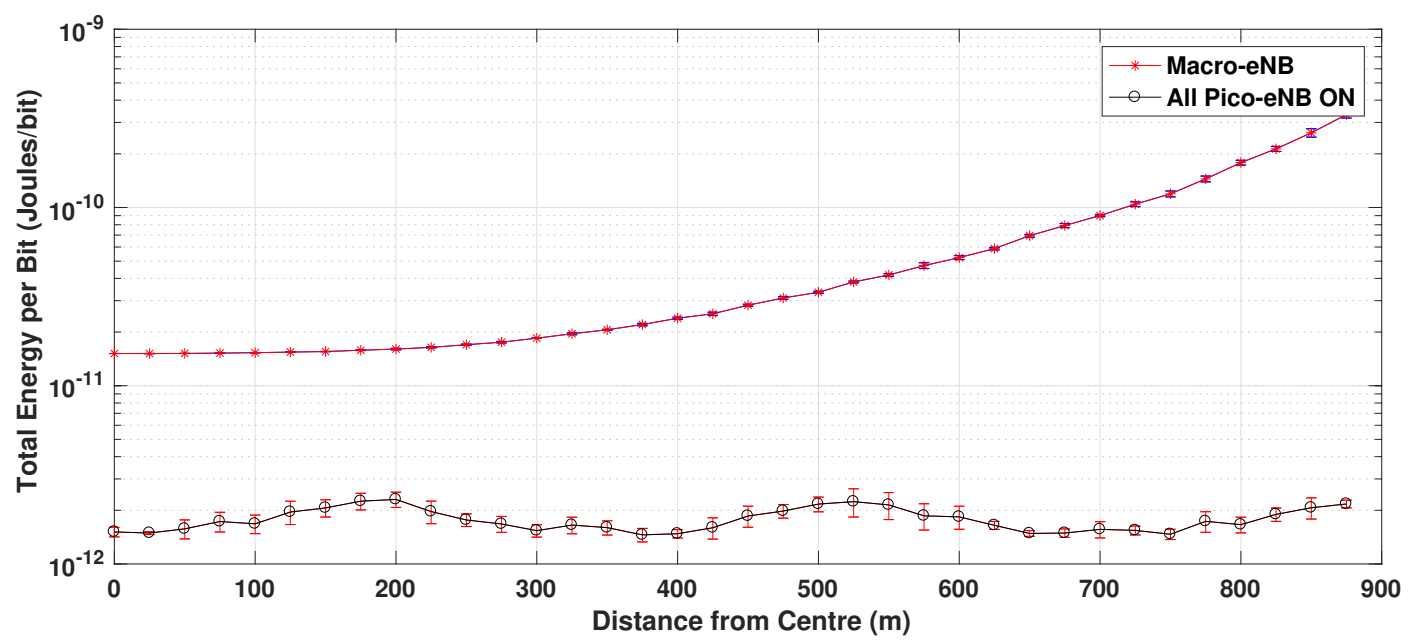

Figure 5.4: TEPB of Macro-eNB vs all Pico-eNBs on for UE moving from centre to the right edge of the network

the base station is kept relatively small.

Finally, the simulation was repeated with the CSA in the EMS/NMS to decide the network setup that provides the best performance, according to the requirements explained in Section 4.4. As we can see from Figures 5.5 and 5.6, the TEPB is improved when the proposed algorithm is used. More precisely, the TEPB fluctuates between 


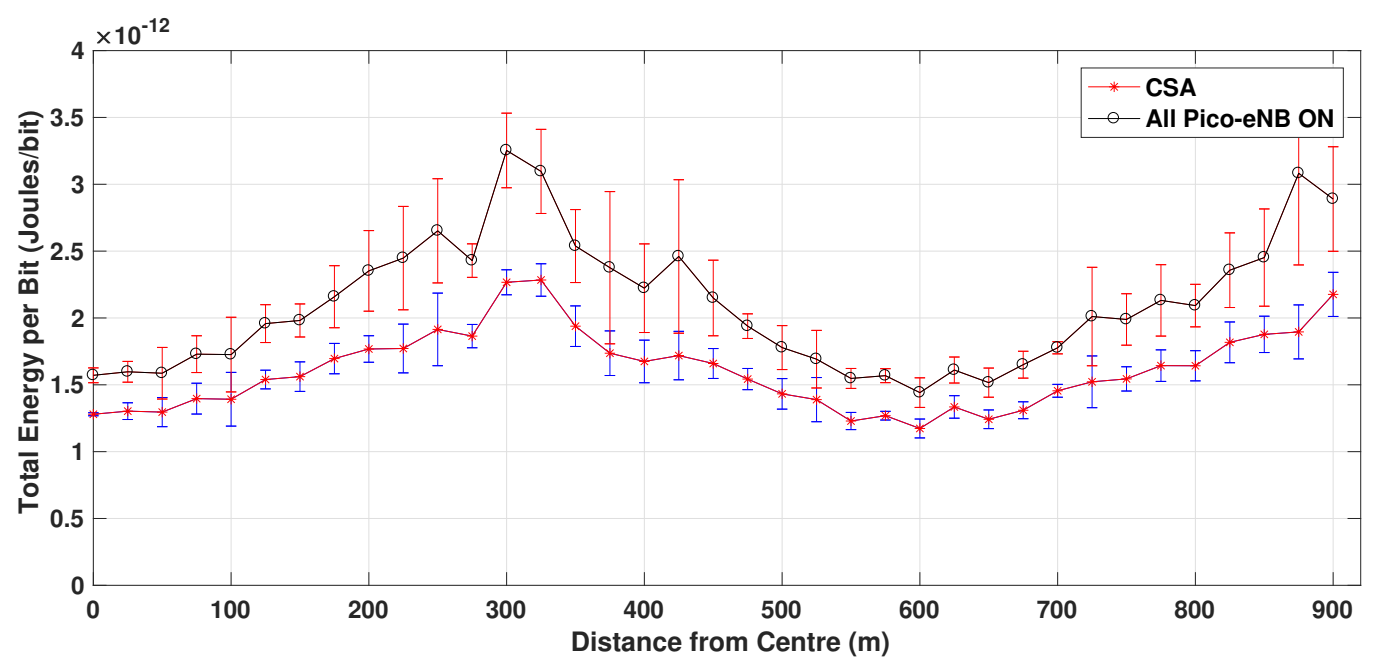

Figure 5.5: Simulation results for UE's centre of distribution moving from centre to the upper edge of the network

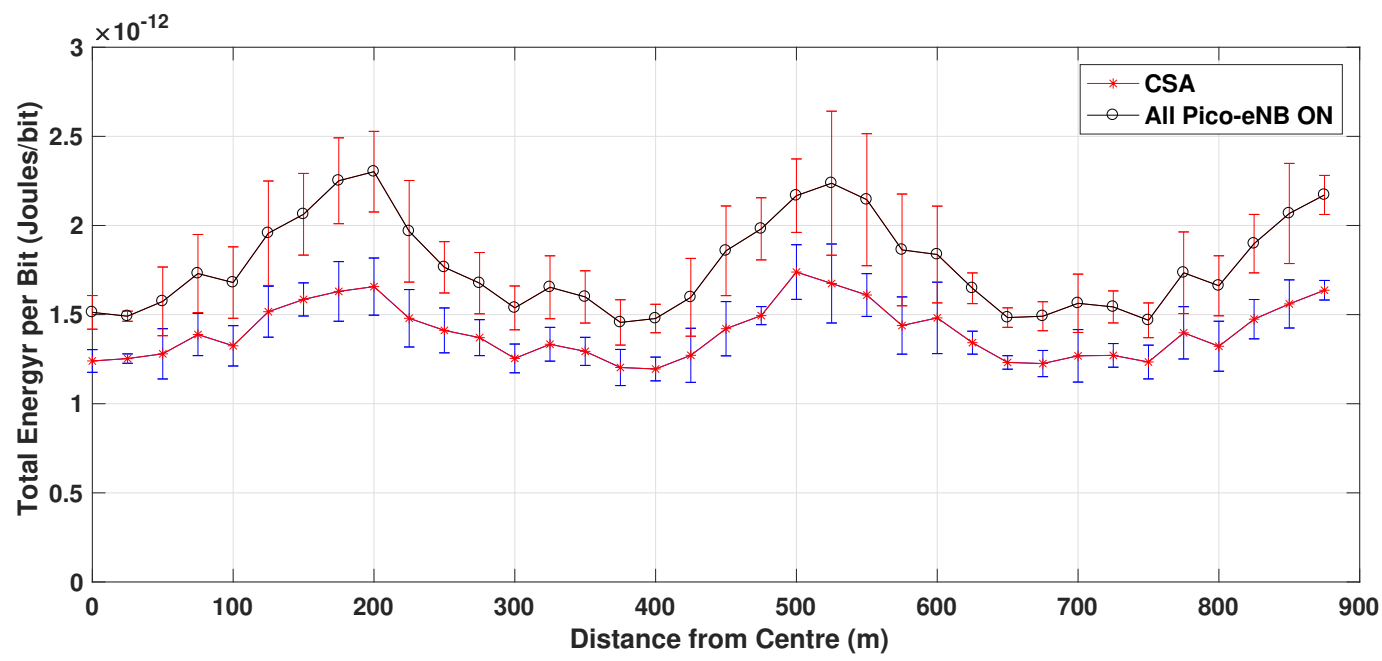

Figure 5.6: Simulation results for UE's centre of distribution moving from centre to the right edge of the network

$1.19 \times 10^{-12}$ and $1.73 \times 10^{-12}$ Joules/bit and $1.17 \times 10^{-12}$ and $2.28 \times 10^{-12}$ Joules/bit when UE move towards the upper and right edge of the network respectively. Comparing Figures 5.5 and 5.6 with Figures 5.3 and 5.4 also show that using the CSA improves the TEPB compared to a network with only one Macro-eNB at its centre. Figure 5.6 also shows a big difference in performance between all Pico-eNBs on and 
Table 5.2: Power consumption for all three scenarios

\begin{tabular}{lc}
\hline Scenario & Average Power Consumption \\
\hline Only Macro-eNB & $1800 \mathrm{~W}$ \\
37 Pico-eNBs ON & $1295 \mathrm{~W}$ \\
CSA & $1120 \mathrm{~W}$ \\
\hline
\end{tabular}

using the CSA between 300 - $325 \mathrm{~m}$ away from the centre of the network. The reason for that difference is the high interference and power consumption caused by having all Pico-eNBs on. Figures 5.3 - 5.6 also show that the 95\% confidence intervals are grouped tightly around the TEPB.

Another advantage of using the CSA is saving power consumption to run these eNBs. Table 5.2 shows that, while using one Macro-eNB, the total power consumption for the network under study is $1800 \mathrm{~W}$. The total network power consumption reduces to $1295 \mathrm{~W}$ when the Macro-eNB is replaced with 37 all on Pico-eNBs. The simulation also shows an improvement in total network power consumption when the CSA is introduced, as the total power consumption becomes $1120 \mathrm{~W}$.

\subsubsection{Evaluation of the Effect of UE Number on the CSA}

To simulate the effect of changing the number of UE throughout the day on the CSA, the simulation was repeated for different numbers of UE, as shown in Figure 5.7. The value of $\mu$ was chosen at the centre of the network, in order to simulate a UE hot spot (downtown during rush hour). 


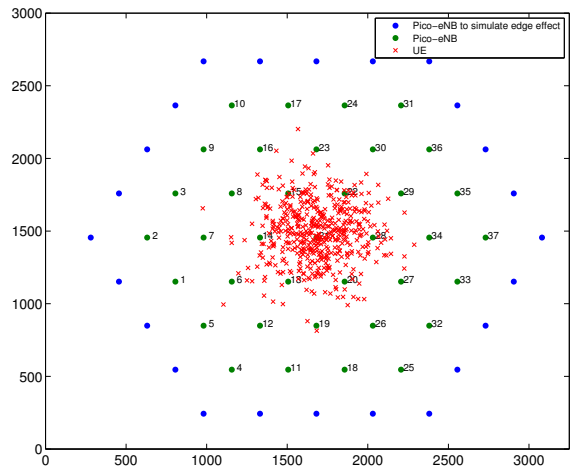

(a) $500 \mathrm{UE}$

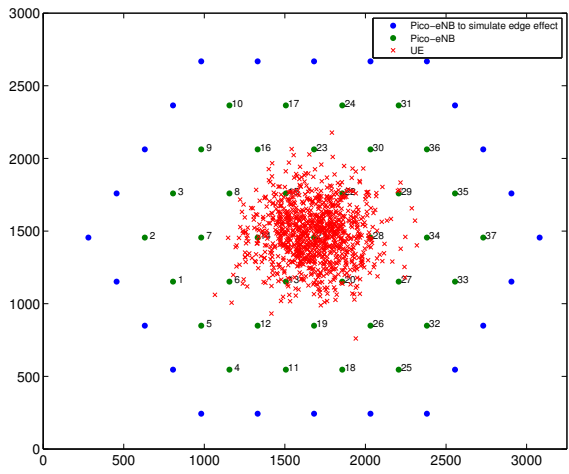

(b) $1000 \mathrm{UE}$

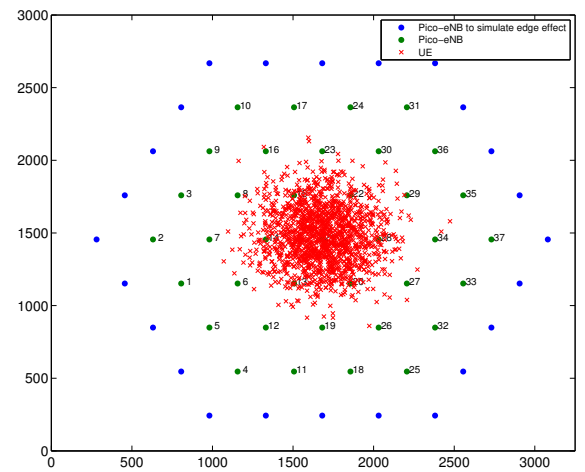

(c) $1500 \mathrm{UE}$

Figure 5.7: Simulation to simulate the effect of the number of UE on the CSA

\subsubsection{Methodology for the Evaluation of the UE Number Effect}

Another simulation was constructed to evaluate the performance of the CSA by changing the number of UE in the network. A similar network setup to the previous scenarios was used in this simulation. The CSA was evaluated for 500, 1000 and $1500 \mathrm{UE}$ which were Gaussian distributed at the centre of the network. The simulation starts with all Pico-eNBs turned on, while the CSA runs in the background. When the CSA finds the best network setup, i.e. which Pico-eNBs to switch on/off, the EMS/NMS starts the switch on/off procedure discussed in Section 4.4. Each simulation takes approximately 30 minutes, so the same simulation was repeated 15 times to generate 


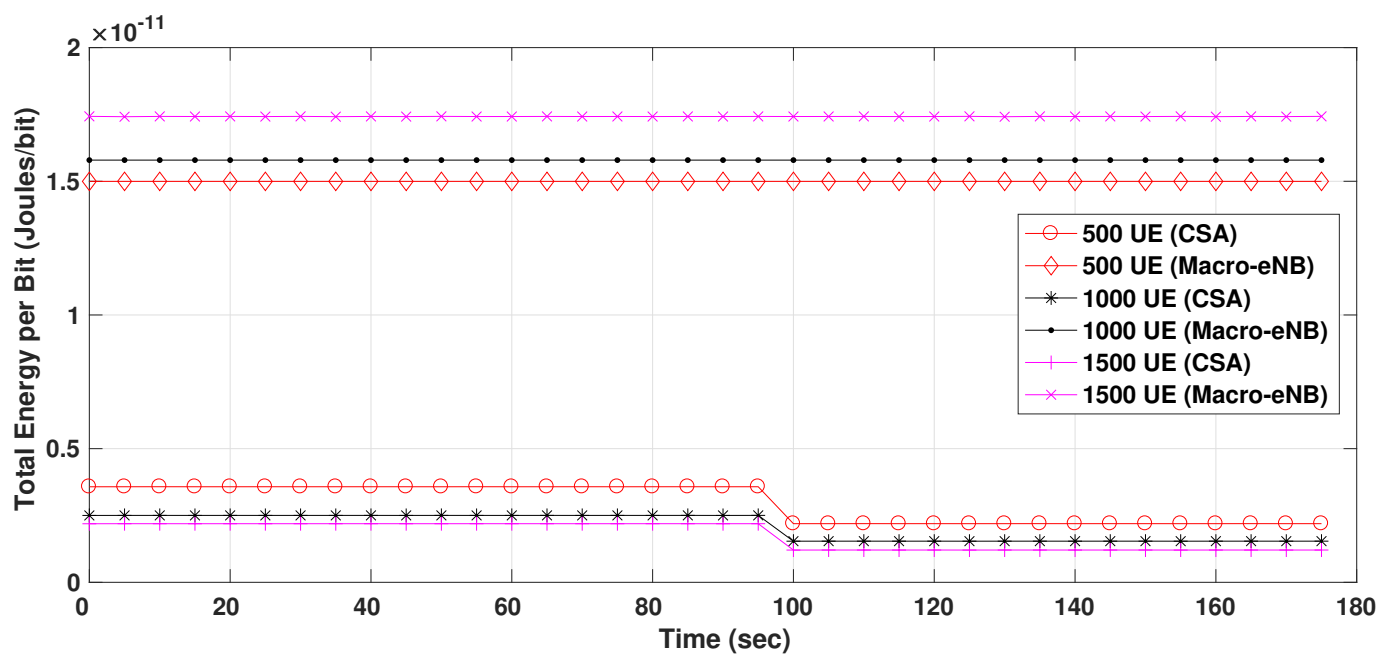

Figure 5.8: Simulation results for 500, 1000 and 1500 UE at the centre of the network

the results of changing the number of UE in the network.

\subsubsection{Results for the Evaluation of the UE Number Effect}

When starting the simulation with all Pico-eNBs on with 500, 1000 and $1500 \mathrm{UE}$ at the centre of the network, the TEPB starts at $3.57 \times 10^{-12}, 2.5 \times 10^{-12}$ and $2.19 \times 10^{-12}$ Joules/bit respectively, as shown in Figure 5.8. At $100 \mathrm{sec}(100,000$ TTI), the CSA decides which Pico-eNBs to switch on/off, then the EMS/NMS applies the network changes. The simulation shows the improvement in the TEPB, as it decreases to $2.19 \times 10^{-12}, 1.54 \times 10^{-12}$ and $1.21 \times 10^{-12}$ Joules/bit for the three values of UE respectively. The decrease in the TEPB is due to the power saved from switching off some Pico-eNBs and the increase in the average network throughput. The latter is caused by the decrease in the interference from the switched off Pico-eNBs. The results were compared with a network with only one Macro-eNB serving the same network area. The TEPB for all three numbers of UE were $14.99 \times 10^{-12}, 15.79 \times 10^{-12}$ and $17.43 \times 10^{-12}$ Joules/bit respectively. The CSA shows a superior performance 
compared to the case of a network with all Pico-eNBs on and the case of a network with only Macro-eNB.

The CSA decision is made by dividing the network into clusters of 7 Pico-eNBs and searching for all possible combinations of all Pico-eNBs in each cluster in order to find which Pico-eNBs to switch on/off. The CSA finds the set of Pico-eNBs that need to be switched on/off to minimize the TEPB, after which the new network setup is applied and Pico-eNBs are switched on/off accordingly. The drawback of such procedure is the processing time, as with the increase in the number of PicoeNBs, the number of possible combinations increases exponentially. Therefore, we proposed another switch on/off algorithm called ECSA, which will be evaluated in the following sections.

\subsection{ECSA Simulation Results and Analysis}

In this section, simulations were constructed to evaluate the performance of the ECSA. As explained in Section 5.1.2, the ECSA starts with all Pico-eNBs on and UE in initial locations. The ECSA searches for a Pico-eNB to switch off, then switches off one Pico-eNB at a time until the ECSA reaches the steady state network setup. Next, the ECSA decides which Pico-eNB to switch on/off based on a snap shot of the new UE locations.

\subsubsection{Simulation Setup}

A network layout with Pico-eNBs was simulated using our system-level simulator. The network is made up of 61 Pico-eNBs (i.e. $N_{e N B s}=61$ ) divided into 4 rings, surrounded by another ring of 30 Pico-eNBs. The outer ring is only used to simulate the edge effect of a network, as shown in Figure 5.9. Pico-eNBs are separated by an ISD of $350 \mathrm{~m}$, although the ECSA can be applied for any Pico-eNB locations in the 


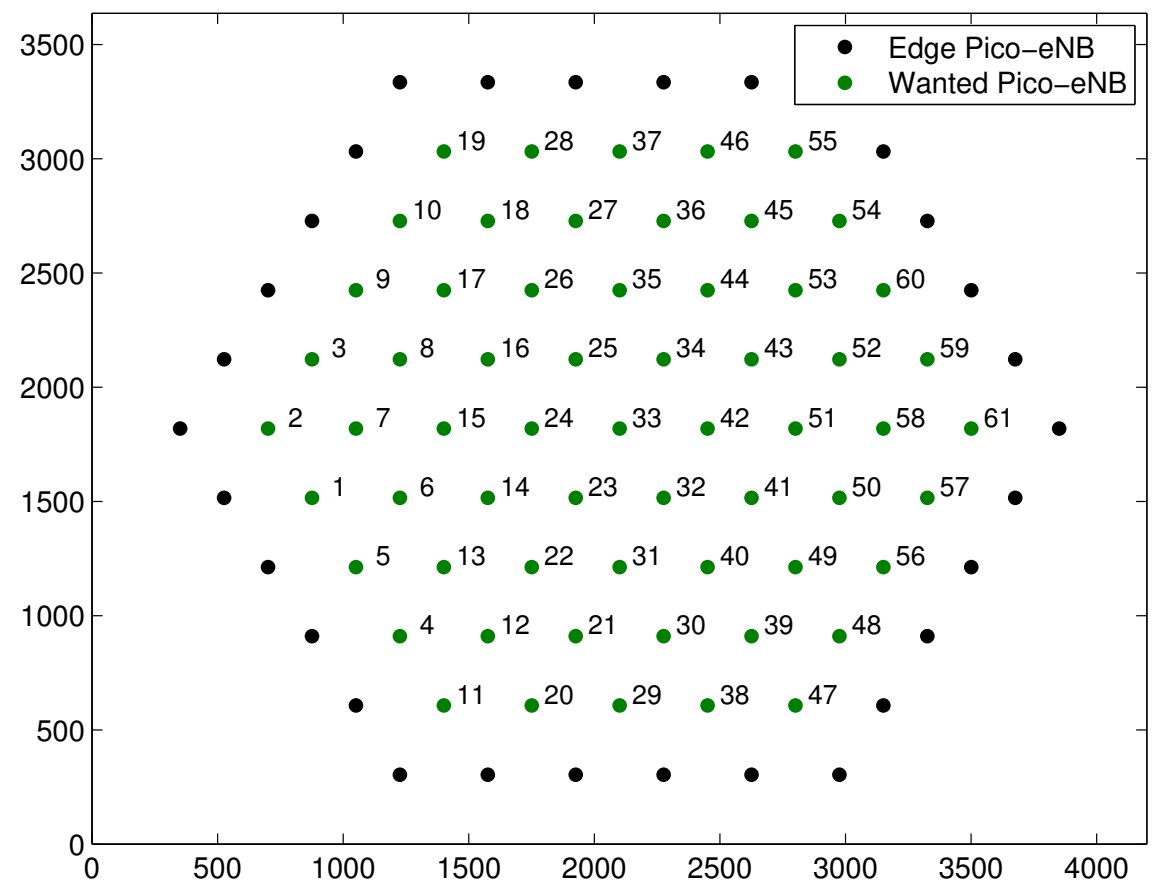

Figure 5.9: E-UTRAN network architecture with only Pico-eNBs

network.

The network topology consists of a standard set of low power Pico-eNBs with omni-directional antennas. Although the ECSA can be applied for any UE distribution, the Manhattan mobility model was used to create urban UE mobility scenarios as explained in Section 4.5.3. Figure 5.10a and Figure 5.10b show an example network setup with 500 and 2000 UE respectively. The simulation parameters are summarized in Table 5.3 .

\subsubsection{Sample Simulation Run}

In this section, an example of a detailed simulation is explained to show how the ECSA behaves over time in order to minimize the TEPB as users are moving within 


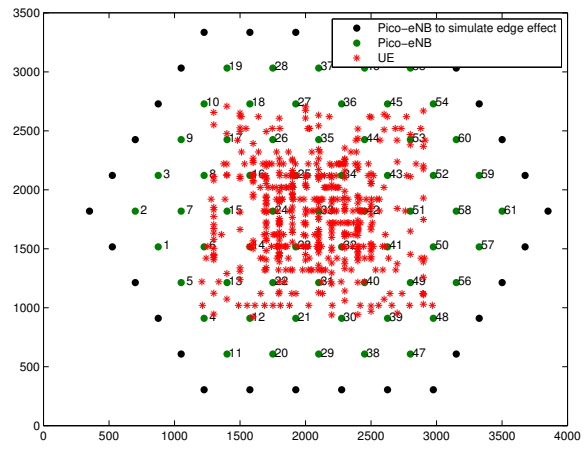

(a) $500 \mathrm{UE}$

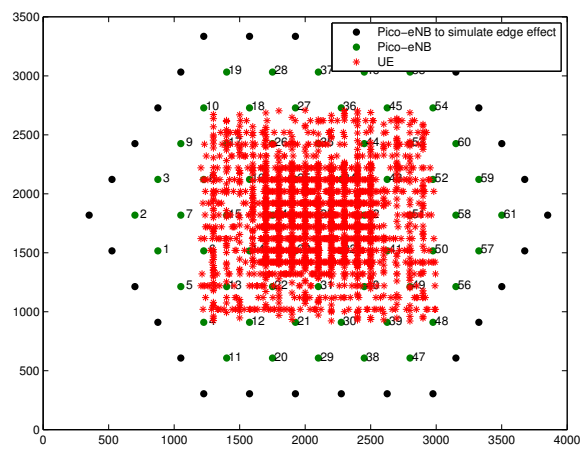

(b) $2000 \mathrm{UE}$

Figure 5.10: Simulation setup for evaluating the effect of the number of UE on the ECSA

Table 5.3: Simulation parameters to evaluate the ECSA

\begin{tabular}{lc}
\hline Parameter & Setting \\
\hline Pico-eNB ISD & $350 \mathrm{~m}$ \\
No. of Pico-eNBs & 61 \\
No. of edge Pico-eNBs & 30 \\
No. of UE & $500,1000,1500,2000$ \\
No. of RBs per UE & 10 \\
Pico-eNB Tx Power & $36 \mathrm{dBm}$ \\
Thermal noise & $-174 \mathrm{dBm} / \mathrm{Hz}$ \\
UE Distribution & Manhattan Mobility Model \\
Bandwidth & MHz at 2 GHz carrier frequency \\
Antenna Height & $15 \mathrm{~m}$ \\
Traffic model & Full buffer, full load \\
\hline
\end{tabular}

the network. A network setup similar to the one shown in Figure 5.9 is used in this example. The simulation starts with all Pico-eNBs on and static UE locations. The 


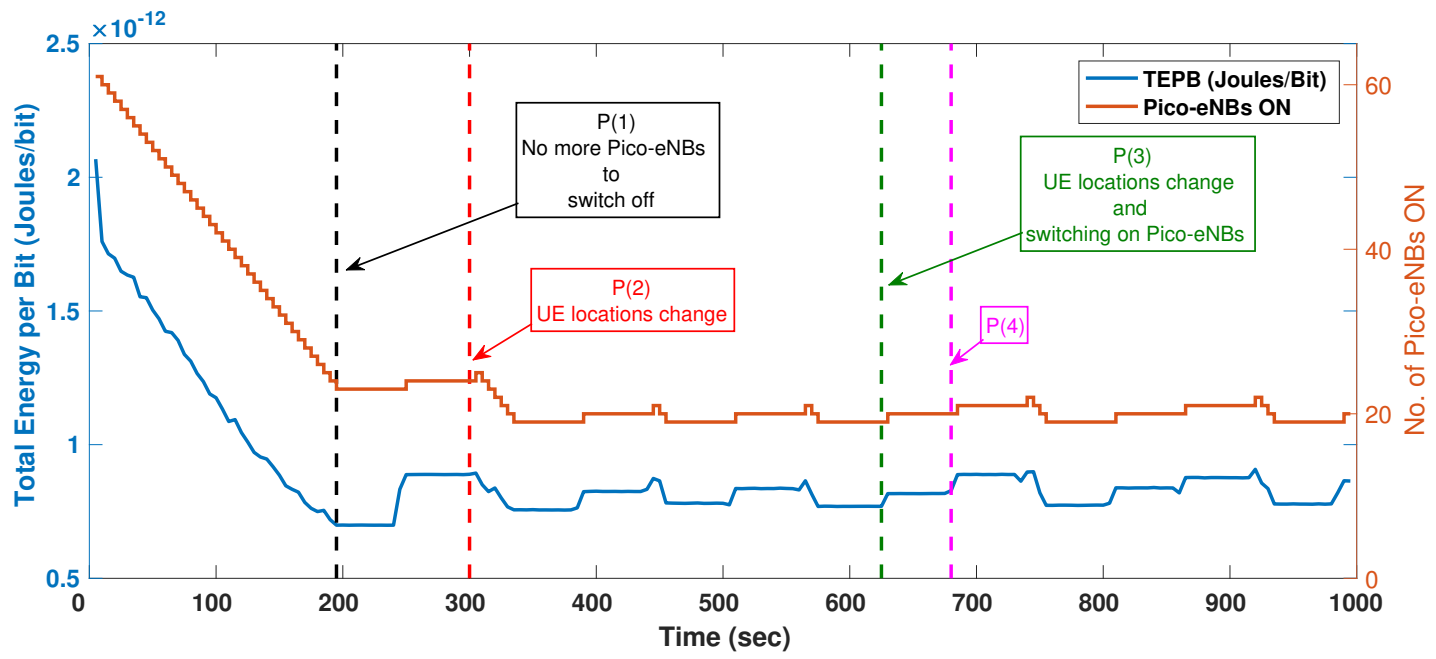

Figure 5.11: Simulation sample for the ECSA for 500 UE

EMS/NMS runs the ECSA to calculate the feasibility of switching on/off a PicoeNB that matches the requirements discussed in Section 4.4. The algorithm keeps switching off one Pico-eNB at a time until it reaches the steady state network setup (see $P(1)$ in Figure 5.11) for that UE distribution. This steady state network setup is the starting point for the ECSA to switch on/off Pico-eNBs based on the movement of UE.

The UE locations is always changing according to the Manhattan mobility model. The EMS/NMS runs the ECSA periodically, every 5 sec (5000 TTI), and takes a snap shot of the UE distribution and checks if a Pico-eNB needs to be switched on/off. At $P(2)$ in Figure 5.11, the UE locations change which causes a decrease in network throughput and in return the TEPB increases. The ECSA accommodates that decrease in throughput by finding a Pico-eNB to switch on. The next time the EMS/NMS runs the ECSA, it decides that a different Pico-eNB needs to be switched off which causes the TEPB to decrease. At $P(3)$, the TEPB increases slightly, which is caused by the change in UE distribution over time. The algorithm finds a Pico-eNB to switch on, which stops the increase in the TEPB, and continues with the same 
network setup until the algorithm runs again at $P(4)$.

This sample simulation showed the steps that the ECSA follows to minimize the TEPB. The ECSA starts with a network with all Pico-eNBs on, then switches off one Pico-eNB at a time to achieve the steady state network setup (minimum TEPB for that UE distribution). After the steady state network setup is achieved, the fluctuations in the TEPB is due to switching on/off Pico-eNBs based on UE movement throughout the network. It can be observed that the TEPB value stays close to the steady state value.

\subsubsection{Evaluation of the Effect of UE Number on the ECSA}

The performance of the ECSA was evaluated by a simulation similar to the one discussed in the previous section. The ECSA was evaluated for 500, 1000, 1500 and 2000 UE randomly distributed in the network.

The simulation runs with all Pico-eNBs turned on, while the ECSA runs in the background. The ECSA keeps switching off Pico-eNBs until it finds the best network setup for the starting UE locations. The ECSA uses the resulting network setup as an initial point, at which it will run the algorithm periodically to switch on/off Pico-eNBs based on the change in UE distribution.

To simulate the change in the number of UE throughout the day, the simulation was repeated for different numbers of UE (500, 1000, 1500, and 2000). Each simulation was run for $800 \mathrm{sec}(800,000$ TTI) and was repeated 20 times.

When starting the simulation with all Pico-eNBs on with 500, 1000, 1500 and 2000 UE initial locations, the network TEPB is $1.93 \times 10^{-12}, 1.67 \times 10^{-12}, 1.64 \times 10^{-12}$, and $1.48 \times 10^{-12}$ Joules $/$ bit respectively. At time $=200 \mathrm{sec}$, the ECSA reaches the steady state network setup for all sets of UE. After the steady state network setup is achieved, the ECSA is ready to adapt the network based on the UE movement (Manhattan mobility model) throughout the day. The fluctuation shown in Figure 5.12 happens 


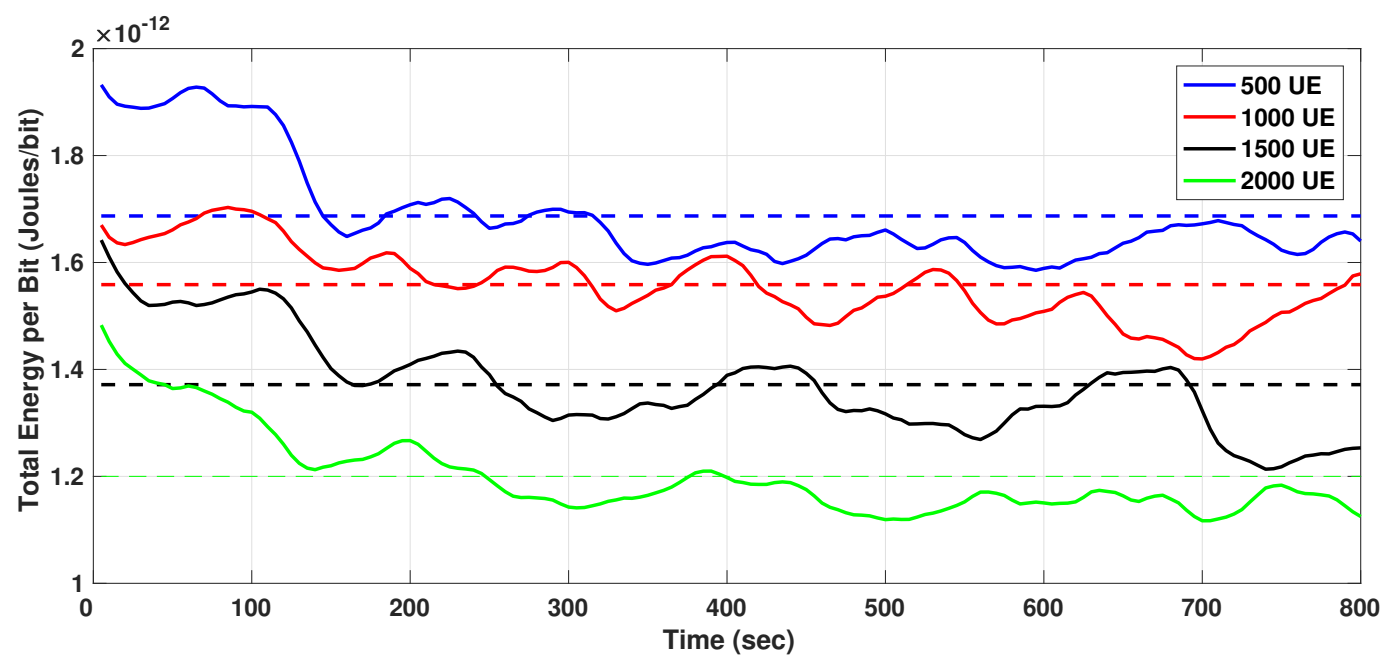

Figure 5.12: Simulation results for 500, 1000, 1500, 2000 UE at the centre of the network

because the ECSA is turning on/off Pico-eNBs to adapt to UE distribution change throughout the simulation. Figure 5.12 also shows the average network TEPB to be $1.69 \times 10^{-12}, 1.56 \times 10^{-12}, 1.37 \times 10^{-12}$ and $1.2 \times 10^{-12}$ Joules/bit for $500,1000,1500$ and 2000 UE respectively.

With lower numbers of UE, some Pico-eNBs can be underutilized but cannot be turned off because they were not able to hand off their UE to any neighboring PicoeNB. This underutilization can lead to a decrease in TEPB with the increase of the number of UE in the network, as seen in Figure 5.12 .

\subsubsection{CSA vs. ECSA}

In order to evaluate the performance of the CSA and ECSA, a simulation that compares both algorithms was performed. A network consists of 37 Pico-eNBs with an ISD of $350 \mathrm{~m}$, and these Pico-eNBs are surrounded with 24 Pico-eNBs to simulate the edge effect of the network as shown in Figure 5.13 .

The CSA was used to search for the best network setup for Gaussian distributed 


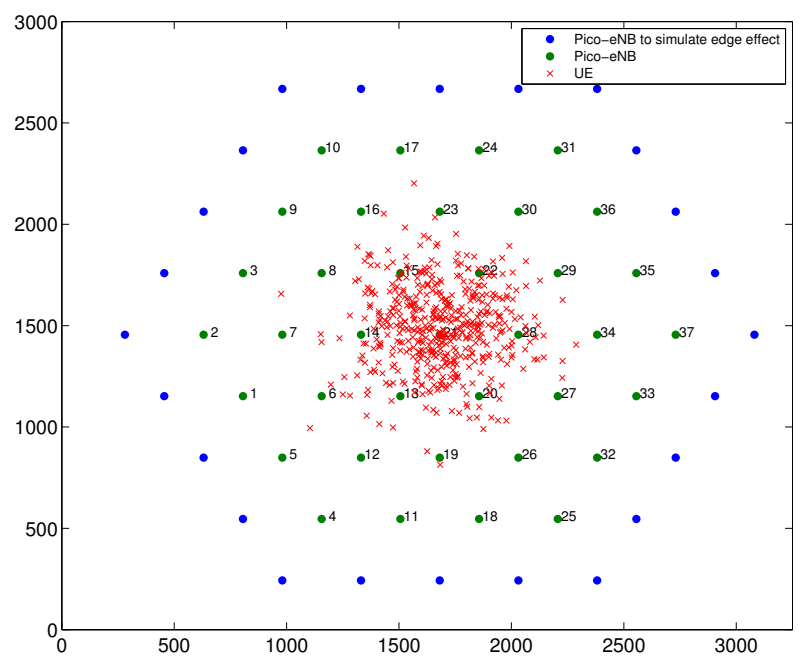

Figure 5.13: Simulation setup for evaluating the performance of CSA and ECSA for $500 \mathrm{UE}$

UE at the centre of the network. The CSA had a time limitation as it had to divide the network into clusters of 7 Pico-eNBs and search for all possible combinations of all Pico-eNBs in each cluster to decide which Pico-eNBs to switch on/off, as explained in Section 5.1.1.

The simulation was repeated for three different scenarios: all Pico-eNBs on, CSA, and ECSA. The first scenario is run without a switch on/off algorithm. For the second and third scenarios, testing the CSA and ECSA, both simulations start with all Pico-eNBs turned on. The CSA runs in the background until it finds the network setup needed for that UE distribution. Then, Pico-eNBs are simultaneously switched on/off to update the network setup. On the other hand, in the third scenario, the ECSA runs in the background and keeps switching off Pico-eNBs until it finds the best network setup to match the UE distribution.

To compare the performance of the CSA and ECSA, a network with 500 stationary UE with Gaussian distribution at the centre of the network was simulated. Each simulation was run for $16 \mathrm{~min}$ and was repeated 20 times. The UE were stationary, 


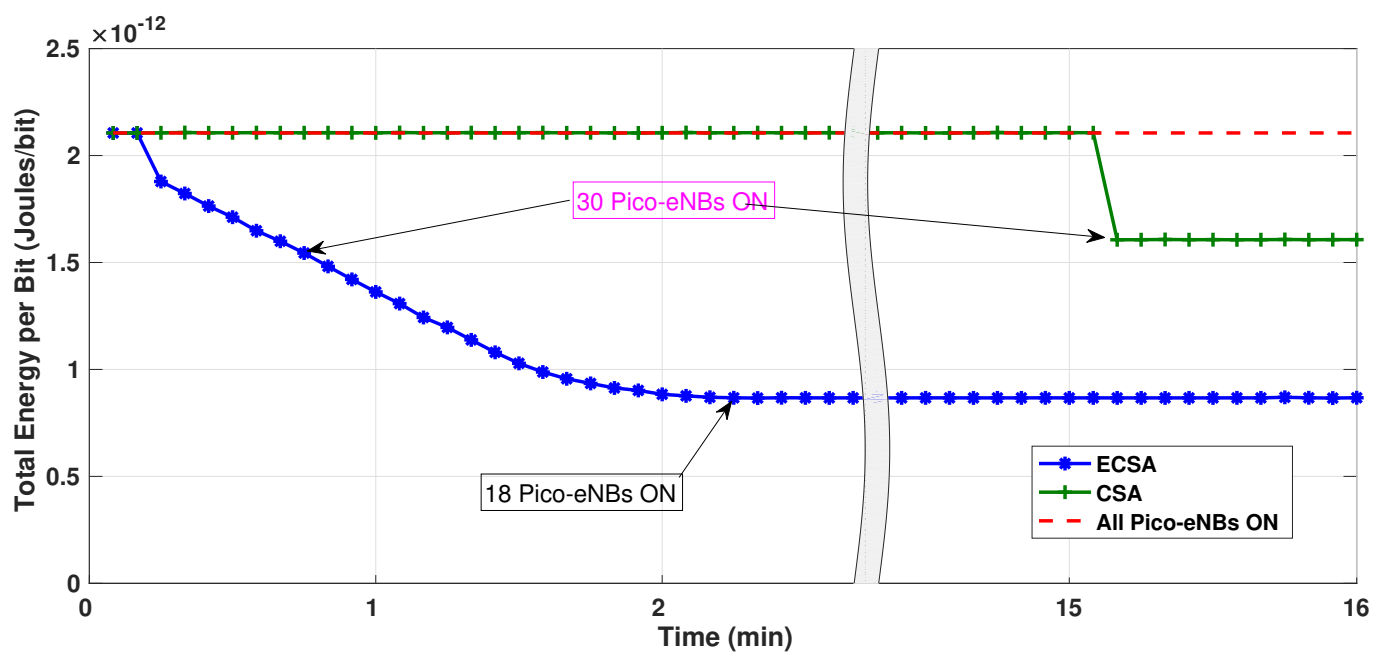

Figure 5.14: Simulation results: CSA vs ECSA

which means that once both algorithms find the set of Pico-eNBs to switch on/off, the network TEPB value will be constant. Furthermore, the CSA processing time is around $15 \mathrm{~min}$. As a result, the simulation was run only for $16 \mathrm{~min}$.

Figure 5.14 shows the network TEPB for all three scenarios. When starting the simulation in all scenarios at time 5 sec and with all Pico-eNBs on, the network TEPB was $2.11 \times 10^{-12}$ Joules/bit. Then for scenario 1 , the TEPB remains constant throughout the simulation since all Pico-eNBs are kept on.

As explained in Section 4.4.2, the CSA requires 15-30 minutes to decide which Pico-eNB to switch on/off. Therefore, in this simulation the CSA scenario was executed and the CSA found the best network setup that matches the UE distribution and simultaneously switched on/off Pico-eNBs according to that. At $15 \mathrm{~min}$, the new network setup runs with 30 Pico-eNBs on and the network TEPB decreases to $1.61 \times 10^{-12}$ Joules/bit.

Finally, in the third scenario, the ECSA runs every 5 sec to find which Pico-eNB to switch on/off to accommodate the current UE distribution. Therefore, at time $10 \mathrm{sec}$, the ECSA searches for which Pico-eNB to switch off and turns it off. At time 
15 sec, the network TEPB starts decreasing until the enhanced algorithm finds the steady state network setup at 140 sec. The steady state network TEPB continues at $0.87 \times 10^{-12}$ Joules/bit with 18 Pico-eNBs on.

In Section 5.3.3 (Figure 5.8), the simulation of a similar network was executed, with only a Macro-eNB at the centre and 500 UE. The TEPB for that network was found to be $15 \times 10^{-12}$ Joules/bit. All the above mentioned scenarios show an improvement in the TEPB of the network compared with a Macro-eNB network. The ECSA was able to switch off more Pico-eNBs than the CSA, 18 and 30 Pico-eNBs remaining on respectively. This difference was due to the way the CSA divides the network into groups of 7 Pico-eNBs, which was explained in Section 5.1.1.

It is worth noting in Figure 5.14 that the processing time of the CSA is greater than the ECSA's. The time axis was broken between 3 and 14 min because the values were constant during that period. This simulation shows that while the CSA is searching for which Pico-eNBs to switch on/off, the ECSA has already reached the steady state and is running at low TEPB. That is another advantage of the ECSA over the CSA. Although it can adapt to sudden changes since the algorithm is run every 5 seconds, the ECSA is considered a static switch on/off algorithm because the time needed for physically switching on/off a Pico-eNB is long (a few minutes). This is significant as by the time the Pico-eNB is physically switched on/off, the UE location might have changed, rendering that decision obsolete.

\subsubsection{ECSA vs. Two Algorithms from Literature}

In this section, we compare the ECSA with two static switch on/off algorithms from the literature. The first algorithm is a greedy-drop algorithm [26] and the second is a greedy-add algorithm [44]. As explained in Section 3.2.2, the decision making in static switch on/off algorithms is done offline and based on a snapshot of UE locations at a certain time. 
The greedy-drop algorithm in [26] starts by assuming that all eNBs are on and that UE are connected to the eNB that provides the best SNR. It is worth noting that the ECSA begins in the same manner but that UE are connected instead to the eNB that provides the best SINR, not SNR. When the greedy-drop algorithm starts, it searches for the eNB with the lowest traffic load and tries to handover UE connected to that eNB to neighboring eNBs. If the handover was successful, then it searches for the next eNB and repeats the same process. The algorithm stops once a UE cannot be handed over (dropped) to any neighboring eNB. The new network setup is applied to the current network and the same process is repeated periodically.

Similar to the greedy-drop algorithm, the greedy-add algorithm in 44 starts with a network in which all eNBs are on and all UE are connected to the eNB that provides the best SNR. That step is necessary to obtain the current load of each eNB and the number of UE connected to it, in order to sort eNBs based on their current load (highest to lowest). Then, the algorithm starts with the assumption that all eNBs are switched off and that all UE are not connected. Then, it searches for the eNB with the highest load and turns it on. UE are connected to that eNB until it reaches its maximum allocated bandwidth. The same process is repeated until all UE are connected to an eNB.

In both algorithms, an outage of $1 \%$ was allowed (similar to the ECSA) and a fixed data rate $(500 \mathrm{kbps})$ was used. Also, they assumed that the spectral efficiency was calculated based on the SNR between the UE and the eNB. Therefore, the bandwidth was calculated with $w=\log _{2}(1+S N R)$. In the ECSA, we calculated the UE throughput using Equation 5.2, which is based on the CQI (which is itself based on SINR) for each UE as discussed in Section 4.3.3. 


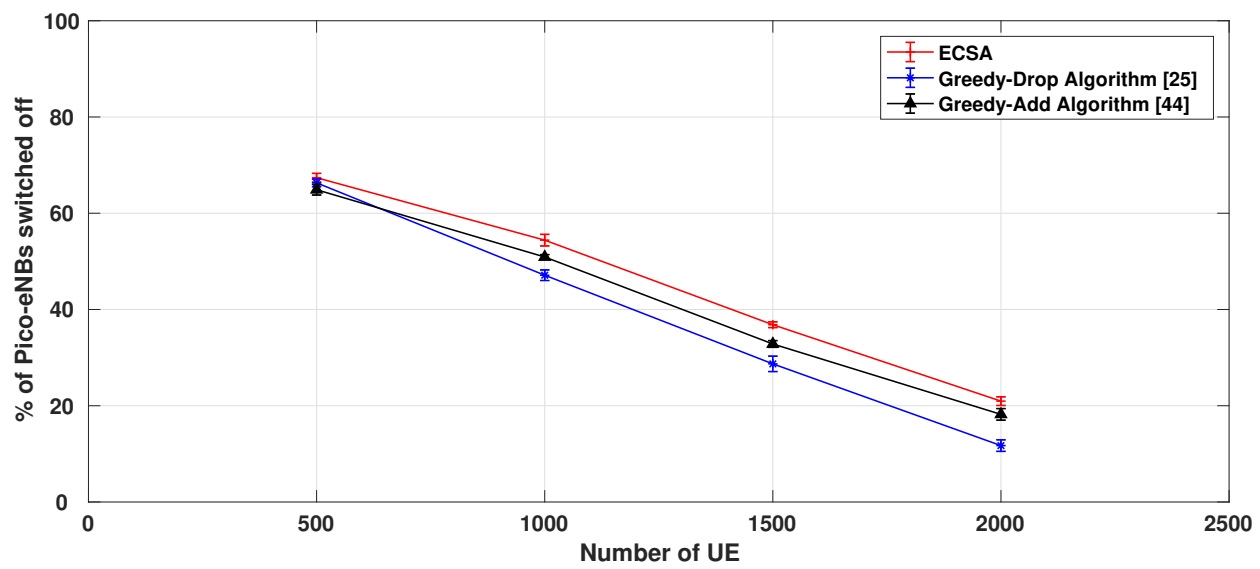

Figure 5.15: Comparison of the $\%$ of Pico-eNBs switched off in the network between the ECSA, the greedy-drop algorithm and the greedy-add algorithm

\subsubsection{Simulation Setup}

The ECSA is compared to the greedy-drop and greedy-add algorithms mentioned above and the results (with $95 \%$ confidence interval) are illustrated in Figure 5.15 and 5.16. To generate the results, a simulation with a network consisting of 61 PicoeNBs that covers $4 \mathrm{~km}^{2}$ was implemented. The simulation was repeated 10 times and was evaluated for 500, 1000, 1500 and 2000 UE uniformly distributed in the network. The simulation parameters are summarized in Table 5.4.

Figure 5.15 presents the percentage of Pico-eNBs switched off in the network for different numbers of UE. For 500 UE in the network, ECSA and greedy-drop algorithms behave similarly. While, the greedy-add algorithm switches off less number of Pico-eNBs. Nevertheless, when the number of UE in the network increases (1000, 1500 and 2000 UE), the proposed algorithm outperforms both algorithms by switching off up more Pico-eNBs (8\% more than greedy-drop algorithm and $4 \%$ more than greedy-add algorithm).

Figure 5.16 presents the network TEPB for all three algorithms. For 500 UE in the network, the ECSA and greedy-drop algorithm outperform the greedy-add 
Table 5.4: Simulation parameters to compare ECSA with greedy-drop and greedyadd algorithms

\begin{tabular}{lc}
\hline Parameter & Setting \\
\hline Pico-eNB ISD & $350 \mathrm{~m}$ \\
No. of Pico-eNBs & 61 \\
No. of edge Pico-eNBs & 30 \\
No. of UE & $500,1000,1500,2000$ \\
No. of RBs per UE & 10 \\
Pico-eNB Tx Power & $36 \mathrm{dBm}$ \\
Thermal noise & $-174 \mathrm{dBm} / \mathrm{Hz}$ \\
UE Distribution & Uniformly distributed \\
Bandwidth & MHz at 2 GHz carrier frequency \\
Antenna Height & $15 \mathrm{~m}$ \\
Traffic model & Full buffer, full load \\
\hline
\end{tabular}

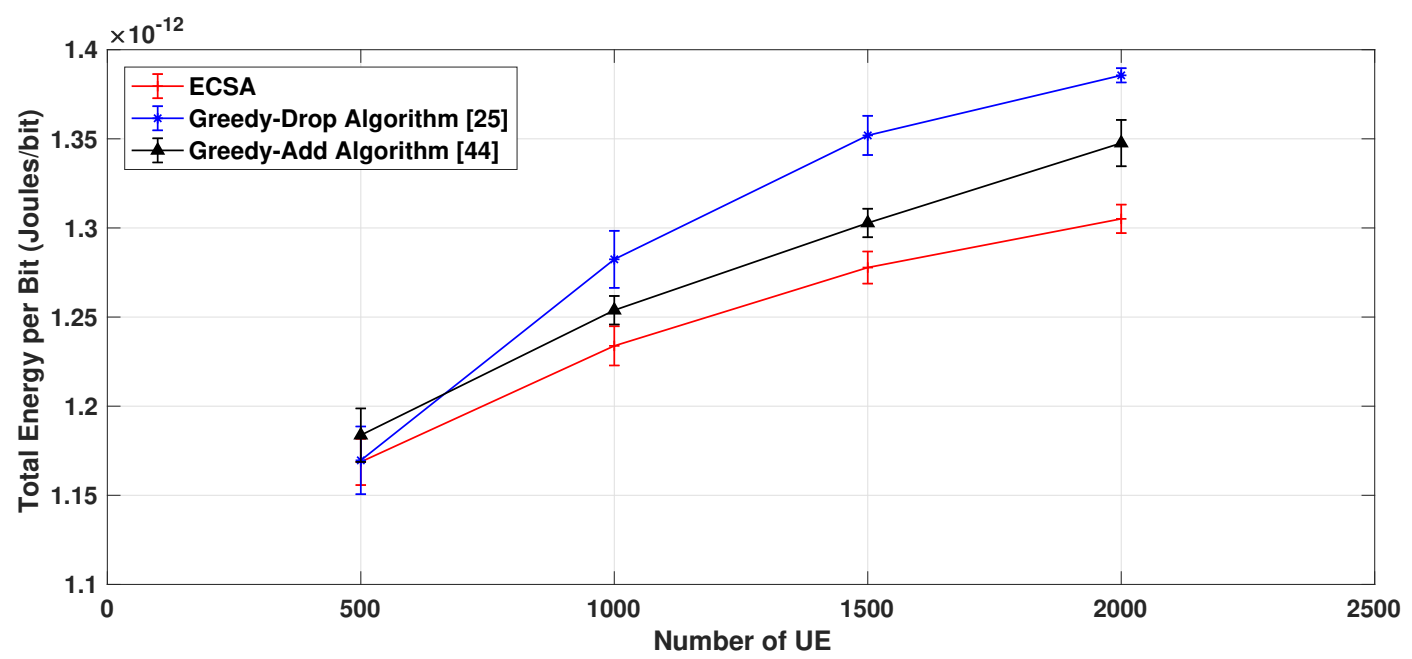

Figure 5.16: Comparison of TEPB of the network between the ECSA, the greedydrop algorithm and the greedy-add algorithm 
algorithm, $1.169 \times 10^{-12}$ Joules/bit compared to $1.184 \times 10^{-12}$ Joules/bit for the greedy-add algorithm. That difference is due to the number of Pico-eNBs switched off by the ECSA and greedy-drop algorithm is slightly larger than the greedy-add algorithm. Furthermore, when the number of UE in the network increases, the ECSA surpasses both algorithms by maintaining a lower TEPB. The better results shown by the ECSA are due to the fact that the algorithm takes into account different UE throughput requirement based on the path-loss between the UE and the Pico-eNB, as discussed in 4.3.1. Another reason for the ECSA's better TEPB is the fact that it was able to switch off more Pico-eNBs, as shown in Figure 5.15. The improvement is due to the fact that both algorithms (greedy-drop and greedy-add) use a fixed data rate (500 kbps) based on the SNR values while in the ECSA, the data rate is calculated based on the CQI (SINR) value for each UE. Furthermore, the improvement is caused by the fundamental approach difference between the three algorithms, as explained in the beginning of this section. 


\section{Chapter 6}

\section{Conclusion and Future Work}

In this chapter, we conclude the thesis and summarize the main contributions and results. Then, we introduce a list of possible future work that can be applied to the current research to further enhance the ECSA.

\subsection{Conclusion}

In this thesis, the idea of adaptive coverage is exploited to reduce power consumption in LTE networks while providing users with the promised high data rates envisioned in LTE networks. As a means of satisfying this growing demand, researchers are trying to solve two important problems: (1) providing the spectrum resources as needed by high data rate users, and (2) supplying the required spectrum at reasonable power consumption in the context of green ICT and profit maximization. The latter is important since service providers are trying to minimize the power required to operate their network. To tackle these two conflicting objectives, a new metric is defined: the total energy per bit (TEPB). This metric takes into consideration both aspects simultaneously.

By observing a network area with a large number of cells that share the same bandwidth over a long period of time, the amount of power used in order to deliver 
a certain number of bits to the users can be measured. The function of the new metric, TEPB, is to help in evaluating/comparing the performance of algorithms and measure the greening of any networks.

In order for Macro-eNBs to deliver high data rates to every spot that they cover, network operators have two options: using higher transmission power, which is impractical, or reducing the distance between the UE and the eNB. Therefore, in this thesis, we proposed as a solution the use of a large number of Pico-eNBs to replace all Macro-eNBs. Simulation shows that, by replacing one Macro-eNB by 37 Pico-eNBs, the network TEPB can be reduced by more than $75 \%$.

To take the proposed vision even further, we designed two centralized switch on/off algorithms (CSA and ECSA) that minimize the TEPB while guaranteeing quality of service. Both proposed switch on/off algorithms determine which Pico-eNB(s) should be switched on/off. Because eNB switching on/off algorithms are considered NP-hard, in this thesis we proposed two implementable heuristic switch on/off algorithms.

The CSA operates by searching for all possible Pico-eNB combinations in the network to find which Pico-eNBs to switch on/off in order to minimize the network TEPB. Simulation shows that, by applying the CSA for the same network setup mentioned earlier (37 Pico-eNBs), the network TEPB can be reduced even further (85\%). The downside of the CSA is the processing time, as the number of combinations increases for a larger number of Pico-eNBs, therefore increasing the processing time. In light of this, we proposed a second switch on/off algorithm called ECSA.

The ECSA starts with all Pico-eNBs on and UE in initial locations. The ECSA then calculates the feasibility of switching off a Pico-eNB. Then, the ECSA switches off one Pico-eNB at a time until it reaches the steady state network setup, meaning that no more Pico-eNBs can be switched off for that specific UE distribution. When the ECSA reaches the steady state network setup, it starts taking snap shots of the moving UE locations. These change based on the type of UE distribution chosen. 
Finally, the ECSA uses these snap shots of UE locations to decide which Pico-eNB to switch on/off in order to reduce the TEPB. Similarly to the CSA, the simulations showed that the ECSA can reduce the TEPB of the network by $90 \%$ compared with the Macro-eNB network mentioned earlier. The drawback of using the ECSA is that the amount of information required by the centralized algorithm is large, specially when the number of Pico-eNBs increases.

With the increase in the number of Pico-eNBs in the network, providing high data rate for high mobility UE becomes an issue. The UE high speed movement causes an increase in the overhead caused by handing over the UE from one Pico-eNB to the neighboring Pico-eNB. Furthermore, the UE periodically reports the CQI value to the serving eNB which means that by the time the value is received and processed by the eNB, it might become invalid. The latter is due to the change in UE location and, in return, can change the CQI of the UE based on the new SINR calculated. Therefore, proper handing over techniques and CQI reporting algorithms need to be implemented for high mobility UE.

In conclusion, replacing Macro-eNBs with a large number of Pico-eNBs combined with switch on/off algorithms minimizes the network TEPB. This helps network operators provide users with the required high data rate while achieving a greener network.

\subsubsection{The Road to 5G Networks}

With the LTE system deployment and it reaching the theoretical limit over the past few years, only gradual improvements and small amounts of new spectrum can be expected with current technologies. Therefore, the industry is looking for new technologies that will be able to handle the increase in the amount of data in wireless networks. That new technology is fifth generation $(5 \mathrm{G})$.

It is expected that $5 \mathrm{G}$ networks, compared to $4 \mathrm{G}$ networks, should achieve $1000 \times$ system capacity, $10 \times$ spectral efficiency, data rate, energy efficiency and close to 
zero delay [92] [93]. The amount of wireless data is expected to increase from under 3 exabytes (EB) in 2010 to over $190 \mathrm{~EB}$ by 2018, and is on track to go over $500 \mathrm{~EB}$ by 2020 [17. Alongside the huge amount of data, the data rates and number of UE are expected to grow exponentially.

Network operators are aiming to connect the entire world with a seamless and ubiquitous communication between anybody, wherever they are, whenever needed by whatever electronic device desired. Therefore, network operators are working on designing next generation network architectures with an infrastructure that is able to support next generation applications and services, i.e., 5G-ready [94].

\subsubsection{The Proposed Vision in 5G}

Because of the huge expected increase in data traffic volume in $5 \mathrm{G}$ networks, the importance of energy efficiency (bits/J) and spectrum efficiency $\left(\right.$ bps $\left./ \mathrm{Hz} / \mathrm{km}^{2}\right)$ will increase 95$]$. For the purpose of $5 \mathrm{G}$ networks to provide the capacity and spectrum efficiency expected, a combination of spatial densification and spectrum aggregation is needed. Spatial densification can be achieved by increasing the number of eNBs in the network and the number of antennas per eNB [96]. Thus, the proposed vision, described in Section 4.1, of adding a large number of Pico-eNBs to the system can help in the spatial densification of the network. Also, the proposed vision can bring the network closer to the users, which works toward increasing the data rate to meet the requirements for $5 \mathrm{G}$ networks. Furthermore, by using Pico-eNBs, the transmit power can be significantly reduced and the same frequency can be reused at near locations, resulting in the improvement of energy and spectrum efficiency simultaneously. Spectrum aggregation refers to a technique that uses discontinuous frequency bands to provide a larger amount of spectrum. The useable spectrum may be increased by aggregating RBs within a given frequency band or in different bands. 


\subsubsection{The Proposed Metric (TEPB) in 5G}

To achieve the demand in $5 \mathrm{G}$ for even higher data rate than in $4 \mathrm{G}$ networks, it is imperative that the energy efficiency of $5 \mathrm{G}$ networks be improved. $5 \mathrm{G}$ relies on a few techniques to provide greener wireless communication, such as massive MIMO, device-to-device communication, beam division multiple access (BDMA) and multiple radio access technologies 97 .

The proposed TEPB metric defined in Section 4.1.1 will be a perfect way of measuring the greening of the network by finding the ratio between the total power consumption in $5 \mathrm{G}$ networks and the number of bits received.

\subsubsection{The ECSA in 5G}

Some changes can be applied to the proposed ECSA in order for it to be applicable in $5 \mathrm{G}$ networks, for example, adding the 5G's expected close to zero delay as a factor in the decision making of the switch on/off algorithm. Also, applying spectrum aggregation for scheduling UE data in the ECSA can increase the data rate of the network, which will play a big role in the selection of which eNB to switch on/off.

\subsection{Future Work}

An important aspect of future work is preparing the ECSA to be implemented in the future $5 \mathrm{G}$ networks. This can be done by further evaluating the performance of the ECSA and reducing the time required by the algorithm to decide which Pico-eNBs need to be switched on/off. Also, as in this thesis we focus only on the downlink communication, it would be of interest to include both downlink and uplink communication jointly in the decision making of the ECSA. Although the results presented here have demonstrated the effectiveness of the proposed switch on/off algorithm, it

could be further evaluated and improved in a number of ways as discussed in the 
following sections.

\subsubsection{Test the ECSA in a Real Network Setup}

The power consumption of each Pico-eNB in the ECSA was assumed to be constant. Therefore, considering more realistic power consumption model for each Pico-eNB that depends on its utilization (as discussed in Section 2.4), the ECSA can achieve more accurate results. Thus, the next step to further evaluate the ECSA would be to test it with real Pico-eNBs. It would be worthwhile to test the ECSA in a real environment to evaluate how it behaves.

\subsubsection{Implement a Distributed Switch On/Off Algorithm}

The complexity of the network increases with the addition of a greater number of Pico-eNBs. The main limitation of the ECSA is the number of Pico-eNBs that can be included under each EMS/MNS. Converting the ECSA from a centralized into a distributed switch on/off algorithm can help reduce the processing time necessary to find which Pico-eNB to switch on/off. With a distributed algorithm, the decision processing is moved from the EMS/NMS to each Pico-eNB, meaning that each Pico-eNB collects its own information as well as neighboring Pico-eNBs' information. Then, the Pico-eNB decides if it needs to be switched on/off accordingly. Therefore, the number of Pico-eNBs in the network would not affect the processing time of the algorithm as much as in the ECSA.

To push the idea of the distributed switch on/off algorithm even further, a dynamic distributed switch on/off algorithm can be implemented. This will allow the switch on/off algorithm to accommodate the fluctuation in UE movement throughout the day. This means the switch on/off algorithm needs to base its decision on live UE information: location, number, channel quality for each UE and traffic load 
requirements per UE. Dynamic switch on/off algorithms are very complex, because processing the network information and adjusting it should be done on the fly. Some techniques can be implemented to allow the dynamic switch on/off, for example UE movement prediction, which allows the algorithm to anticipate future UE locations that a UE might cross [98]. In turn, that helps in calculating the channel quality (SINR) of the connection between the eNB and the UE connected to it.

The main problem with dynamic switch on/off algorithms is the time needed for the eNB to switch from off to on mode (which depends on the physical eNB used), as by the time the eNB is switched on, the UE information has changed and the algorithm's decision might be invalid at this point. Therefore, this aspect needs to be taken into consideration in the dynamic switch on/off algorithm.

\subsubsection{Include Backhauling in the Switch On/Off Algorithm}

As mentioned in Section 6.1.1, network operators are evolving towards $5 \mathrm{G}$ networks. The spread of small BS architecture is considered a key enabling technique for the emerging 5G. With the miniaturization of BSs, they can now be installed almost anywhere, such as on lamp posts, bus stops, billboards and even in manholes, where existing landline infrastructure is available. Ericsson developed advanced street lighting systems that include Ericsson zero sites, which is an implementation of $5 \mathrm{G}$ small BSs [99].

However, the downside of this approach is that, with a larger number of Pico-eNBs, providing an economical and ubiquitous backhaul connectivity to the core network is going to be a challenging aspect of the proposed vision, as explained in Section 4.1.3. For that reason, a proper network planning is needed.

In [100], they provided an overview of different backhaul solutions and challenges related to backhauling small BS in 5G networks. The current ESCA does not include the effect of switching on/off Pico-eNBs on the power consumption of the core. 
Including the power consumption of the core in the decision of the switch on/off algorithm can be another extension to this research.

Implementing a switch on/off algorithm that takes into consideration all elements of the network in its decision making is an important step towards the transition to 5G networks, especially with dense Pico-eNB networks.

\subsubsection{Adding New Pico-eNB When Needed}

Even the most stable networks sometimes need the addition of new eNBs to accommodate an unexpected failure of one of more eNBs or an increase in demand for coverage. The ECSA can be used (with a few tweaks) to help network operators plan where to add new Pico-eNBs as needed. The ECSA can also be evaluated for the following two cases:

\subsubsection{New Pico-eNBs in events}

Usually in big events, such as stadiums during big sporting events, cellular networks fail to accommodate the sudden increase in user demand. By adding temporary PicoeNBs during these events, it is possible to reduce the connection failure and dropped calls rate and increase QoS.

\subsubsection{New Pico-eNBs in emergencies}

Many emergency responders continue to have significant challenges to communicate via radio on a daily basis. This fact becomes more apparent in disasters, for example floods or earthquakes [101]. With this in mind, Canadian Interoperability Technology Interest Group (CITIG) is exploring the possibility of creating $700 \mathrm{MHz}$ LTE in a box [102]. One of the possible LTE in a box applications is to install a temporary Pico-eNB in order to create a wireless bubble allowing emergency responders to 
communicate during search and rescue missions. This can be viewed as providing elastic coverage, by combining fixed and transportable Pico-eNBs (including unmanned aerial vehicle (UAV)-based Pico-eNBs or other mobile Pico-eNBs). 


\section{Appendix A}

\section{SINR Distribution Calculation Proof}

Each eNB transmits a power $P_{t x_{i}}$ so the power received by a UE is characterized by a lognormal distribution.

$$
\begin{gathered}
P_{r x}\left(d_{i}\right)=P_{t x_{i}} c d_{i}^{-\eta} f_{i} \\
f_{i}=10^{\frac{\varepsilon}{10}} \\
\ln \left(P_{r x}\left(d_{i}\right)\right)=\ln \left(P_{t x_{i}} c d_{i}^{-\eta}\right)+\ln \left(f_{i}\right)
\end{gathered}
$$

Because its a lognormal random variable, then it has zero mean so $\epsilon=0$

$$
\ln \left(P_{r x}\left(d_{i}\right)\right)=a\left[\frac{1}{a} \ln \left(P_{t x_{i}} c d_{i}^{-\eta}\right)\right]
$$

If we assume $\mathrm{c}=1$ then we can calculate the individual mean power received with:

$$
m_{i}=\frac{1}{a} \ln \left(P_{t x_{i}} d_{i}^{-\eta}\right)
$$

From Fenton-Wilkinson method, the total mean of lognormal random variables is:

$$
m=\ln \left[\sum_{i} e^{\left(m_{i}+\frac{\sigma_{i}^{2}}{2}\right)}\right]-\frac{\sigma_{t}^{2}}{2}
$$


The total mean power received can be calculated with:

$$
a m=\ln \left[\sum_{i} e^{\left.\left(\ln \left(P_{t x_{i}}\right)-\eta \ln \left(d_{i}\right)\right)+\frac{a^{2} \sigma_{i}^{2}}{2}\right)}\right]-\frac{a^{2} \sigma_{t}^{2}}{2}
$$

Since $P_{t x_{i}}=P_{t x}$ for what ever eNB and considering identical $\sigma_{i}=\sigma$ :

$$
a m=\ln \left(P_{t x}\right)+\frac{a^{2} \sigma^{2}}{2}+\ln \left(\sum_{i} e^{\left(-\eta \ln \left(d_{i}\right)\right)}\right)-\frac{a^{2} \sigma_{t}^{2}}{2}
$$

We can calculate the mean interference power by:

$$
\ln \left(\overline{I_{\text {int }}}\right)=\ln \left(P_{t x}\right)+\frac{a^{2} \sigma^{2}}{2}+\ln \left(\sum_{i} d_{i}^{-\eta}\right)-\frac{a^{2} \sigma_{t}^{2}}{2}
$$

From Fenton-Wilkinson method, the total variance of lognormal random variables is:

$$
\sigma_{t}^{2}=\ln \left(\frac{\sum_{i} \sigma_{i}^{2}}{\left(\sum_{i} \mathbb{E}(i)\right)^{2}}+1\right)
$$

Where:

$$
\begin{gathered}
\sigma_{i}^{2}=\left(e^{2 m_{i}+\sigma_{i}^{2}}\right)\left(e^{\sigma_{i}^{2}}-1\right) \\
\mathbb{E}(j)=\left(e^{m_{i}+\frac{\sigma_{i}^{2}}{2}}\right)
\end{gathered}
$$

Therefore, the variance of the sum of power received is:

$$
\begin{gathered}
a^{2} \sigma_{t}^{2}=\ln \left(\frac{\sum_{i} a^{2} \sigma^{2}}{\left(\sum_{i} a \mathbb{E}(j)\right)^{2}}+1\right) \\
a^{2} \sigma_{t}^{2}=\ln \left[\frac{\sum_{i} e^{\left(2 a m_{i}+a^{2} \sigma^{2}\right)}\left(e^{a^{2} \sigma^{2}}-1\right)}{\left(\sum_{i} e^{\left(a m_{i}+\frac{a^{2} \sigma^{2}}{2}\right)}\right)^{2}}+1\right]
\end{gathered}
$$

Assuming that:

$$
G(d, \eta)=\frac{\sum_{i} d_{i}^{-2 \eta}}{\left(\sum_{i} d_{i}^{-\eta}\right)^{2}}
$$


Then the total mean interference is:

$$
\begin{gathered}
\ln \left(\overline{I_{\text {int }}}\right)=\ln \left(P_{t x}\right)+\ln \left(\sum_{i} d_{i}^{-\eta}\right)+\frac{a^{2} \sigma^{2}}{2}-\frac{a^{2} \sigma_{t}^{2}}{2} \\
\overline{I_{\text {int }}}=P_{t x} \sum_{i} d_{i}^{-\eta}\left(e^{\frac{a^{2} \sigma^{2}}{2}-\frac{a^{2} \sigma_{t}^{2}}{2}}\right)
\end{gathered}
$$

Substituting $a^{2} \sigma_{t}^{2}=\ln (X)$ equation in here:

$$
\begin{gathered}
\overline{I_{\text {int }}}=P_{t x} \sum_{i} d_{i}^{-\eta}\left(e^{\frac{a^{2} \sigma^{2}}{2}-\frac{\ln (X)}{2}}\right) \\
\overline{I_{\text {int }}}=P_{t x} \sum_{i} d_{i}^{-\eta}\left[\frac{e^{\frac{a^{2} \sigma^{2}}{2}}}{\left.e^{\ln (X)^{1 / 2}}\right]}\right. \\
\overline{I_{\text {int }}}=P_{t x} \sum_{i} d_{i}^{-\eta} e^{\frac{a^{2} \sigma^{2}}{2}} X^{-1 / 2} \\
\overline{I_{\text {int }}}=P_{t x} \sum_{i} d_{i}^{-\eta} e^{\frac{a^{2} \sigma^{2}}{2}}\left(G(d, \eta)\left(e^{a^{2} \sigma^{2}}-1\right)+1\right)^{\frac{1}{2}} \\
a^{2} \sigma_{t}^{2}=\ln \left(G(d, \eta)\left(e^{a^{2} \sigma^{2}}-1\right)+1\right)+\ln \left(e^{a^{2} \sigma^{2}}\right)
\end{gathered}
$$

The logarithmic mean of the ration between the interference and desired power:

$$
m_{f}=\frac{\overline{I_{i n t}}}{\overline{I_{d e s}}}
$$

Because $P_{t x}$ is the same for all eNBs then:

$$
\overline{I_{\text {int }}}=\frac{\sum_{i} d_{i}^{-\eta}}{d_{i}^{-\eta}} e^{\frac{a^{2} \sigma^{2}}{2}}\left(G(d, \eta)\left(e^{a^{2} \sigma^{2}}-1\right)+1\right)^{\frac{1}{2}}
$$

Denoting:

$$
H(d, \sigma)=e^{a^{2} \sigma^{2} / 2}\left(G(d, \eta)\left(e^{a^{2} \sigma^{2}}-1\right)+1\right)^{\frac{1}{2}}
$$


and

$$
y_{f}(d, \eta)=\frac{\sum_{i} d_{i}^{-\eta}}{d^{-\eta}}
$$

We get:

$$
m_{f}=y_{f}(d, \eta) H(d, \sigma)
$$

In $\mathrm{dB}$, we can express it:

$$
m_{f}=\frac{1}{a} \ln \left(y_{f}(d, \eta) H(d, \sigma)\right)
$$

The standard deviation is given by:

$$
a^{2} s_{f}^{2}=a^{2} \sigma_{t}^{2}+a^{2} \sigma^{2}
$$

Therefore, we get:

$$
\begin{aligned}
& a^{2} s_{f}=2\left(a^{2} \sigma^{2}-\ln H(d, \sigma)\right) \\
& s_{f}=2\left(\sigma^{2}-\frac{1}{a^{2}} \ln (H(d, \sigma))\right)
\end{aligned}
$$




\section{References}

[1] 3rd Generation Partnership Project, "Radio frequency (RF) system scenarios," (3GPP), Tech. Rep. TR 36.942, April 2010.

[2] M. Deruyck, W. Vereecken, E. Tanghe, W. Joseph, M. Pickavet, L. Martens, and P. Demeester, "Comparison of power consumption of mobile WiMAX, HSPA and LTE access networks," in Proc. CTTE'10, 2010, pp. 1-7.

[3] H. Zarrinkoub, Understanding LTE with MATLAB: From Mathematical Modeling to Simulation and Prototyping, 1st ed. Wiley Publishing, 2014.

[4] G. Auer, O. Blume, V. Giannini, I. Godor, M. A. Imran, Y. Jading, E. Katranaras, M. Olsson, D. Sabella, P. Skillermark, and W. Wajda, "Energy efficiency analysis of the reference systems, areas of improvements and target breakdown," EARTH, Tech. Rep. INFSO-ICT-247733, December 2010.

[5] K. Son, S. Chong, and G. D. Veciana, "Dynamic association for load balancing and interference avoidance in multi-cell networks," IEEE Transactions on Wireless Communications, vol. 8, no. 7, pp. 3566-3576, July 2009.

[6] H. Hu, J. Zhang, X. Zheng, Y. Yang, and P. Wu, "Self-configuration and selfoptimization for LTE networks," IEEE Communications Magazine, vol. 48, no. 2, pp. $94-100$, february 2010.

[7] M. R. Garey and D. S. Johnson, Computers and Intractability; A Guide to the Theory of NP-Completeness. New York, NY, USA: W. H. Freeman \& Co., 1990.

[8] F. Han, Z. Safar, W. S. Lin, Y. Chen, and K. J. R. Liu, "Energy-efficient cellular network operation via base station cooperation," in 2012 IEEE International Conference on Communications (ICC), June 2012, pp. 4374-4378.

[9] A. Ghosh, J. Zhang, J. G. Andrews, and R. Muhamed, Fundamentals of LTE, 1st ed. $\quad$ Upper Saddle River, NJ, USA: Prentice Hall Press, 2010. 
[10] A. Ghosh, R. Ratasuk, B. Mondal, N. Mangalvedhe, and T. Thomas, "Lteadvanced: next-generation wireless broadband technology [invited paper]," IEEE Wireless Communications, vol. 17, no. 3, pp. 10-22, June 2010.

[11] 3rd Generation Partnership Project, "Overview of 3GPP release 12," (3GPP), Tech. Rep. V0.0.9, Jun. 2013.

[12] H. Hamdoun, P. Loskot, T. OÄôFarrell, and J. He, "Survey and applications of standardized energy metrics to mobile networks," annals of telecommunications, vol. 67, no. 3-4, pp. 113-123, 2012.

[13] I. T. Union, "Guidelines for evaluation of radio interface technologies for IMTAdvanced," ITU-R, Tech. Rep. ITU-R M.2135-1, Dec. 2009.

[14] K. Johansson, A. Furuskar, P. Karlsson, and J. Zander, "Relation between base station characteristics and cost structure in cellular systems," in Proc. PIMRC'04, vol. 4, 2004, pp. 2627-2631 Vol.4.

[15] M. Etoh, T. Ohya, and Y. Nakayama, "Energy consumption issues on mobile network systems," in Proc. SAINT'08, 2008, pp. 365-368.

[16] Z. Hasan, H. Boostanimehr, and V. Bhargava, "Green cellular networks: A survey, some research issues and challenges," IEEE Communications Surveys Tutorials, vol. 13, no. 4, pp. 524-540, 2011.

[17] J. G. Andrews, S. Buzzi, W. Choi, S. V. Hanly, A. Lozano, A. C. K. Soong, and J. C. Zhang, "What will $5 \mathrm{~g}$ be?" IEEE Journal on Selected Areas in Communications, vol. 32, no. 6, pp. 1065-1082, June 2014.

[18] M. Z. Asghar, S. Hamalainen, and N. Meinke, "Experimental system for selfoptimization of LTE networks," in Proc. MSWiM'12, ser. PM2HW2N '12. New York, NY, USA: ACM, 2012, pp. 91-98.

[19] H. Holma and A. Toskala, LTE for UMTS : Evolution to LTE-Advanced, 2nd ed. Hoboken, NJ, USA: Wiley, 2010.

[20] 3rd Generation Partnership Project, "Self-configuration of network elements; concepts and requirements (release 12)," (3GPP), Tech. Rep. TS 32.501 V12.1.0, Dec. 2013.

[21] — - "Self-organizing networks (SON) policy network resource model (NRM) integration reference point (IRP) requirements (release 11)," (3GPP), Tech. Rep. TS 32.521 V11.1.0, Dec. 2012. 
[22] — , "Self-organizing networks (SON); self-healing concepts and requirements (release 12)," (3GPP), Tech. Rep. TS 32.541 V12.0.0, Oct. 2014.

[23] — " "3GPP work items on self-organizing networks," (3GPP), Tech. Rep. v0.0.9, Sep. 2012.

[24] Z. Niu, Y. Wu, J. Gong, and Z. Yang, "Cell zooming for cost-efficient green cellular networks," IEEE Communications Magazine, vol. 48, no. 11, pp. 7479, November 2010.

[25] Ł. Budzisz, F. Ganji, G. Rizzo, M. A. Marsan, M. Meo, Y. Zhang, G. Koutitas, L. Tassiulas, S. Lambert, B. Lannoo, M. Pickavet, A. Conte, I. Haratcherev, and A. Wolisz, "Dynamic resource provisioning for energy efficiency in wireless access networks: A survey and an outlook," IEEE Communications Surveys Tutorials, vol. 16, no. 4, pp. 2259-2285, Fourthquarter 2014.

[26] F. Alaca, A. Sediq, and H. Yanikomeroglu, "A genetic algorithm based cell switch-off scheme for energy saving in dense cell deployments," in Proc. GC Workshops'12, Dec 2012, pp. 63-68.

[27] S. Zhou, J. Gong, Z. Yang, Z. Niu, and P. Yang, "Green mobile access network with dynamic base station energy saving," in Proc. ACM Mobicom'09, 2009, pp. 1-5.

[28] A. Yildiz, T. Girici, and H. Yanikomeroglu, "A pricing based algorithm for cell switching off in green cellular networks," in 2013 IEEE r7th Vehicular Technology Conference (VTC Spring), June 2013, pp. 1-6.

[29] E. Oh, K. Son, and B. Krishnamachari, "Dynamic base station switching-on/off strategies for green cellular networks," IEEE Transactions on Wireless Communications, vol. 12, no. 5, pp. 2126-2136, May 2013.

[30] A. Bousia, E. Kartsakli, L. Alonso, and C. Verikoukis, "Dynamic energy efficient distance-aware base station switch on/off scheme for lte-advanced," in 2012 IEEE Global Communications Conference (GLOBECOM), Dec 2012, pp. 1532 1537.

[31] A. S. Alam, L. S. Dooley, and A. S. Poulton, "Traffic-and-interference aware base station switching for green cellular networks," in Proc. CAMAD'13, Sept 2013, pp. 63-67. 
[32] W. G. Siyi Wang, "Energy and cost implications of a traffic aware and qualityof-service constrained sleep mode mechanism," IET Communications, vol. 7, pp. 2092-2101(9), December 2013.

[33] M. A. Marsan, L. Chiaraviglio, D. Ciullo, and M. Meo, "Switch-off transients in cellular access networks with sleep modes," in Proc. ICC'11, June 2011, pp. $1-6$.

[34] J. Lorincz, A. Capone, and D. Begusic, "Impact of service rates and base station switching granularity on energy consumption of cellular networks," EURASIP Journal on Wireless Communications and Networking, vol. 2012, no. 1, 2012. [Online]. Available: http://dx.doi.org/10.1186/1687-1499-2012-342

[35] J. Gong, S. Zhou, Z. Niu, and P. Yang, "Traffic-aware base station sleeping in dense cellular networks," in Proc. IWQoS'10, June 2010, pp. 1-2.

[36] K. C. Tun and K. Kunavut, "An overview of cell zooming algorithms and power saving capabilities in wireless networks," KMUTNB: International Journal of Applied Science and Technology, vol. 7, pp. 1-13, 2014.

[37] S. Kokkinogenis and G. Koutitas, "Dynamic and static base station management schemes for cellular networks," in 2012 IEEE Global Communications Conference (GLOBECOM), Dec 2012, pp. 3443-3448.

[38] D. G. G, H. Yanikomeroglu, M. Garcia-Lozano, and S. R. Boqué, "A novel multiobjective framework for cell switch-off in dense cellular networks," in 2014 IEEE International Conference on Communications (ICC), June 2014, pp. 2641-2647.

[39] A. Kumar and C. Rosenberg, "Energy and throughput trade-offs in cellular networks using base station switching," IEEE Transactions on Mobile Computing, vol. PP, no. 99, pp. 1-1, 2015.

[40] I. Aydin, H. Yanikomeroglu, and Ü. Aygölü, "User-aware cell switch-off algorithms," in 2015 International Wireless Communications and Mobile Computing Conference (IWCMC), Aug 2015, pp. 1236-1241.

[41] A. Bousia, A. Antonopoulos, L. Alonso, and C. Verikoukis, "Green; distanceaware base station sleeping algorithm in lte-advanced," in Proc. ICC'12, June 2012, pp. 1347-1351. 
[42] X. Weng, D. Cao, and Z. Niu, "Energy-efficient cellular network planning under insufficient cell zooming," in 2011 IEEE 73rd Vehicular Technology Conference (VTC Spring), May 2011, pp. 1-5.

[43] K. Abdallah, I. Cerutti, and P. Castoldi, "Energy-efficient coordinated sleep of LTE cells," in Proc. ICC'12, 2012, pp. 5238-5242.

[44] T. Beitelmal and H. Yanikomeroglu, "A set cover based algorithm for cell switch-off with different cell sorting criteria," in 2014 IEEE International Conference on Communications Workshops (ICC), June 2014, pp. 641-646.

[45] L. Chiaraviglio, M. Mellia, and F. Neri, "Energy-aware umts core network design," in Proc. WPMC'08, 2008, pp. 1-5.

[46] L. Chiaraviglio, D. Ciullo, M. Meo, and M. Marsan, "Energy-efficient management of umts access networks," in Proc. ITC'09, Sept 2009, pp. 1-8.

[47] M. Marsan, L. Chiaraviglio, D. Ciullo, and M. Meo, "Optimal energy savings in cellular access networks," in Proc. ICC Workshops'09, June 2009, pp. 1-5.

[48] A. Ghosh, N. Mangalvedhe, R. Ratasuk, B. Mondal, M. Cudak, E. Visotsky, T. A. Thomas, J. G. Andrews, P. Xia, H. S. Jo, H. S. Dhillon, and T. D. Novlan, "Heterogeneous cellular networks: From theory to practice," IEEE Communications Magazine, vol. 50, no. 6, pp. 54-64, June 2012.

[49] C. Liu, B. Natarajan, and H. Xia, "Small cell base station sleep strategies for energy efficiency," IEEE Transactions on Vehicular Technology, vol. PP, no. 99, pp. 1-1, 2015.

[50] A. Damnjanovic, J. Montojo, Y. Wei, T. Ji, T. Luo, M. Vajapeyam, T. Yoo, O. Song, and D. Malladi, "A survey on 3gpp heterogeneous networks," IEEE Wireless Communications, vol. 18, no. 3, pp. 10-21, June 2011.

[51] H. Claussen, L. Ho, and F. Pivit, "Effects of joint macrocell and residential picocell deployment on the network energy efficiency," in Proc. PIMRC'08, Sept 2008, pp. 1-6.

[52] T. Q. S. Quek, W. C. Cheung, and M. Kountouris, "Energy efficiency analysis of two-tier heterogeneous networks," in Proc. 17th European Wireless'11, April 2011, pp. 1-5. 
[53] A. Saleh, O. Bulakci, S. Redana, B. Raaf, and J. Hamalainen, "Evaluating the energy efficiency of lte-advanced relay and picocell deployments," in Proc. WCNC'12, April 2012, pp. 2335-2340.

[54] I. Ashraf, F. Boccardi, and L. Ho, "Power savings in small cell deployments via sleep mode techniques," in Proc. PIMRC Workshops'10, Sept 2010, pp. 307-311.

[55] — - "Sleep mode techniques for small cell deployments," IEEE Communications Magazine, vol. 49, no. 8, pp. 72-79, August 2011.

[56] Q. Wang and J. Zheng, "A distributed base station on/off control mechanism for energy efficiency of small cell networks," in Proc. ICC'15, June 2015, pp. 3317-3322.

[57] Y. Fu, Z. Fei, C. Xing, J. Kuang, and L. Wan, "Semi-dynamic switch on/off algorithm over multi-pico stations in heterogeneous network," in 2012 International Conference on Wireless Communications and Signal Processing (WCSP), Oct 2012, pp. 1-5.

[58] Y. S. Soh, T. Quek, M. Kountouris, and H. Shin, "Energy efficient heterogeneous cellular networks," IEEE Journal on Selected Areas in Communications, vol. 31, no. 5, pp. 840-850, May 2013.

[59] R. Sappidi, S. Mosharrafdehkordi, C. Rosenberg, and P. Mitran, "Planning for small cells in a cellular network: Why it is worth it," in Proc. WCNC'14, April 2014, pp. 2277-2282.

[60] J. Wu, S. Jin, L. Jiang, and G. Wang, "Dynamic switching off algorithms for pico base stations in heterogeneous cellular networks," EURASIP Journal on Wireless Communications and Networking, vol. 2015, no. 1, p. 117, 2015. [Online]. Available: http://dx.doi.org/10.1186/s13638-015-0280-y

[61] R. Torrea-Duran, C. Desset, S. Pollin, and A. Dejonghe, "Adaptive energy efficient scheduling algorithm for lte pico base stations," in Proc. FutureNetw'12, July 2012, pp. 1-8.

[62] H. Klessig, A. Fehske, and G. Fettweis, "Energy efficiency gains in interferencelimited heterogeneous cellular mobile radio networks with random micro site deployment," in Proc. 34th IEEE Sarnoff Symposium'11, May 2011, pp. 1-6. 
[63] F. Richter, A. Fehske, and G. Fettweis, "Energy efficiency aspects of base station deployment strategies for cellular networks," in Proc. VTC'09-Fall, Sept 2009, pp. 1-5.

[64] F. Richter and G. Fettweis, "Cellular mobile network densification utilizing micro base stations," in Proc. ICC'10, May 2010, pp. 1-6.

[65] F. Richter, A. Fehske, P. Marsch, and G. Fettweis, "Traffic demand and energy efficiency in heterogeneous cellular mobile radio networks," in Proc. VTC'10Spring, May 2010, pp. 1-6.

[66] C. Khirallah, J. Thompson, and D. Vukobratovic, "Energy efficiency of heterogeneous networks in lte-advanced," in Proc. WCNCW'12, April 2012, pp. $53-58$.

[67] A. Mukherjee, S. Bhattacherjee, S. Pal, and D. De, "Femtocell based green power consumption methods for mobile network," Computer Networks, vol. 57, no. 1, pp. $162-178,2013$.

[68] L. Saker, S. Elayoubi, and T. Chahed, "How femtocells impact the capacity and the energy efficiency of lte-advanced networks," in Proc. PIMRC'11, Sept 2011, pp. 177-181.

[69] L. Saker, S. Elayoubi, R. Combes, and T. Chahed, "Optimal control of wake up mechanisms of femtocells in heterogeneous networks," IEEE Journal on Selected Areas in Communications, vol. 30, no. 3, pp. 664-672, April 2012.

[70] S. Xu, J. Han, and T. Chen, "Enhanced inter-cell interference coordination in heterogeneous networks for lte-advanced," in Proc. VTC'12-Spring, May 2012, pp. 1-5.

[71] S. Mukherjee, "Distribution of downlink sinr in heterogeneous cellular networks," IEEE Journal on Selected Areas in Communications, vol. 30, no. 3, pp. 575-585, April 2012.

[72] L. Suárez, L. Nuaymi, and J.-M. Bonnin, "Energy-efficient $\{B S\}$ switching-off and cell topology management for macro/femto environments," Computer Networks, vol. 78, pp. 182 - 201, 2015, special Issue: Green Communications. [Online]. Available: http://www.sciencedirect.com/science/article/pii/S1389128614004162 
[73] W. Vereecken, L. Haratcherev, M. Deruyck, W. Joseph, M. Pickavet, L. Martens, and P. Demeester, "The effect of variable wake up time on the utilization of sleep modes in femtocell mobile access networks," in Proc. WONS'12, Jan 2012, pp. 63-66.

[74] N. S. Networks, "Outdoor 3G/LTE small cells deployment strategy - the race to the pole," Nokia Siemens Networks, Tech. Rep., 2013, white paper. [Online]. Available: http://nsn.com/sites/default/files/document/ 20130623_outdoor_small_cells_white_paper_final.pdf

[75] Lemko, "Lemko LTE pico technical specifications." [Online]. Available: http://www.lemko.com/pdf/Lemko_Pico_Technical_Specifications.pdf

[76] J. G. Andrews, H. Claussen, M. Dohler, S. Rangan, and M. C. Reed, "Femtocells: Past, present, and future," IEEE Journal on Selected Areas in Communications, vol. 30, no. 3, pp. 497-508, April 2012.

[77] J. Hoadley and P. Maveddat, "Enabling small cell deployment with hetnet," IEEE Wireless Communications, vol. 19, no. 2, pp. 4-5, April 2012.

[78] D. Lopez-Perez, I. Guvenc, G. de la Roche, M. Kountouris, T. Q. S. Quek, and J. Zhang, "Enhanced intercell interference coordination challenges in heterogeneous networks," IEEE Wireless Communications, vol. 18, no. 3, pp. 22-30, June 2011.

[79] J. K. Karlof, Integer Programming: Theory and Practice. CRC Press, 2006.

[80] 3rd Generation Partnership Project, "FDD base station (BS) classification," (3GPP), Tech. Rep. TR 25.951, May 2011.

[81] T. Rappaport, Wireless Communications: Principles and Practice, 2nd ed. Upper Saddle River, NJ, USA: Prentice Hall PTR, 2001.

[82] S. Lee and K. Huang, "Coverage and economy of cellular networks with many base stations," IEEE Communications Letters, vol. 16, pp. 1038-1040, 2012.

[83] J.-S. Ferenc and Z. Neda, "On the size distribution of Poisson-Voronoi cells," Physica A-Statistical Mechanics And Its Applications, vol. 385, no. 2, pp. 518$526,2007$.

[84] Y. Wu, R. H. Y. Louie, M. R. McKay, and I. B. Collings, "Generalized framework for the analysis of linear mimo transmission schemes in decentralized wireless ad hoc networks," IEEE Transactions on Wireless Communications, vol. 11, no. 8, pp. 2815-2827, August 2012. 
[85] J. M. Kelif and M. Coupechoux, "Joint impact of pathloss shadowing and fast fading - an outage formula for wireless networks," CoRR, vol. abs/1001.1110, 2010. [Online]. Available: http://arxiv.org/abs/1001.1110

[86] L. Fenton, "The sum of log-normal probability distributions in scatter transmission systems," IRE Transactions on Communications Systems, vol. 8, no. 1, pp. 57-67, March 1960.

[87] M. Haenggi and R. K. Ganti, "Interference in large wireless networks," Found. Trends Netw., vol. 3, no. 2, pp. 127-248, Feb. 2009. [Online]. Available: http://dx.doi.org/10.1561/1300000015

[88] C. Li, J. Zhang, and K. B. Letaief, "Throughput and energy efficiency analysis of small cell networks with multi-antenna base stations," IEEE Transactions on Wireless Communications, vol. 13, no. 5, pp. 2505-2517, May 2014.

[89] F. Martinez, J.-C. Cano, C. Calafate, and P. Manzoni, "Citymob: A mobility model pattern generator for vanets," in Proc. ICC Workshops'08, May 2008, pp. $370-374$.

[90] C. Bettstetter, "Smooth is better than sharp: A random mobility model for simulation of wireless networks," in Proc. MSWiM'01, ser. MSWIM '01. New York, NY, USA: ACM, 2001, pp. 19-27. [Online]. Available: http://doi.acm.org/10.1145/381591.381600

[91] J. C. Ikuno, M. Wrulich, and M. Rupp, "System level simulation of LTE networks," in Proc. VTC'10-Spring, Taipei, Taiwan, May 2010.

[92] M. Ruffini, "Multidimensional convergence in future 5g networks," Journal of Lightwave Technology, vol. 35, no. 3, pp. 535-549, Feb 2017.

[93] S. Zhang, X. Xu, Y. Wu, and L. Lu, "5g: Towards energy-efficient, low-latency and high-reliable communications networks," in 2014 IEEE International Conference on Communication Systems, Nov 2014, pp. 197-201.

[94] C. X. Wang, F. Haider, X. Gao, X. H. You, Y. Yang, D. Yuan, H. M. Aggoune, H. Haas, S. Fletcher, and E. Hepsaydir, "Cellular architecture and key technologies for $5 \mathrm{~g}$ wireless communication networks," IEEE Communications Magazine, vol. 52, no. 2, pp. 122-130, February 2014.

[95] F. Adachi, "Wireless network evolution toward 5g network," in 2016 21st OptoElectronics and Communications Conference (OECC) held jointly with 2016 International Conference on Photonics in Switching (PS), July 2016, pp. 1-3. 
[96] H. Bogucka, P. Kryszkiewicz, and A. Kliks, "Dynamic spectrum aggregation for future $5 \mathrm{~g}$ communications," IEEE Communications Magazine, vol. 53, no. 5, pp. 35-43, May 2015.

[97] A. Abrol and R. K. Jha, "Power optimization in 5g networks: A step towards green communication," IEEE Access, vol. 4, pp. 1355-1374, 2016.

[98] P. S. Prasad and P. Agrawal, "Movement prediction in wireless networks using mobility traces," in 2010 rth IEEE Consumer Communications and Networking Conference, Jan 2010, pp. 1-5.

[99] Ericsson. (2017, April) Reusing existing infrastructure for new small cell sites. [Online]. Available: https://www.ericsson.com/networks/topics/invisible-sites

[100] M. Jaber, M. A. Imran, R. Tafazolli, and A. Tukmanov, "5g backhaul challenges and emerging research directions: A survey," IEEE Access, vol. 4, pp. 17431766, 2016.

[101] N. Nikaein and D. Câmara, Wireless Public Safety Networks. Elsevier Science, 11 2015, vol. 1.

[102] DGEPS, "Public safety canada response to industry canada's consultation on a policy and technical framework for the $700 \mathrm{mhz}$ band and aspects related to commercial mobile spectrum," Public Safety Canada, Tech. Rep. SMSE-018-10, February 28, 2011. 